\title{
Multiple Binding Modes of Inhibitors to Carbonic Anhydrases: How to Design Specific Drugs Targeting 15 Different Isoforms?
}

Vincenzo Alterio, ${ }^{\dagger}$ Anna Di Fiore ${ }^{\dagger}$ Katia D’Ambrosio, ${ }^{\dagger}$ Claudiu T. Supuran, ${ }^{*}$, and Giuseppina De Simone* ${ }^{\dagger}$

${ }^{\dagger}$ Istituto di Biostrutture e Bioimmagini-CNR, via Mezzocannone 16, 80134 Napoli, Italy

${ }^{\ddagger}$ Università degli Studi di Firenze, Laboratorio di Chimica Bioinorganica, Rm. 188, Via della Lastruccia 3, I-50019 Sesto Fiorentino, Firenze, Italy

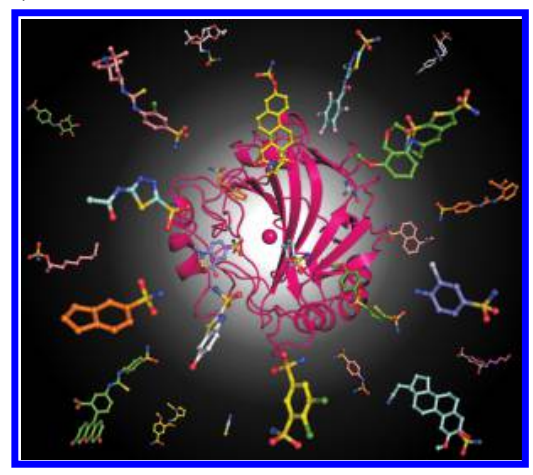

\section{CONTENTS}

1. Introduction

2. Structural Features of $\alpha$-CAs

3. Insights into $\mathrm{CA}$ Catalytic Mechanism: $\mathrm{CO}_{2}$ and $\mathrm{HCO}_{3}{ }^{-}$Binding to hCA II

4. Insights into CA Inhibition: Structural Features of Zinc Binding Inhibitors

4.1. Binding of Ureates and Hydroxamates

4.2. Thiol Derivatives

4.3. Metal-Complexing Anions

4.4. Sulfonamides

4.4.1. Benzenesulfonamides

4.4.2. Thiophene, Thiadiazole, and Thiadiazoline Derivatives

4.4.3. Sulfonamides Containing Other Ring Systems

4.4.4. Thiazide Diuretics

4.4.5. Aliphatic Sulfonamides

4.5. Sulfamates and Sulfamides

4.5.1. Aliphatic Sulfamates

4.5.2. Sulfamate CAls Also Acting as Steroid Sulfatase and Aromatase Inhibitors

4.6. Sulfonamides/Sulfamates/Sulfamides Containing Sugar Moieties

5. Insights into CA Inhibition: Structural Features of Non-Zinc-Binding Inhibitors

5.1. Compounds Anchoring to the Zinc Bound Water Molecule

5.1.1. Phenols

5.1.2. Spermine and Related Polyamines

5.2. Compounds Located at the Entrance of the Active Site: Coumarins and Lacosamide

6. Conclusions

Author Information
Corresponding Author

Notes

4461

Biographies

Acknowledgments

Abbreviations

References

4461

4461

4462

4462

4462

\section{INTRODUCTION}

Carbonic anhydrases (CAs, EC 4.2.1.1) are ubiquitous metalloenzymes, present throughout most living organisms and encoded by five evolutionarily unrelated gene families: the $\alpha$-, $\beta$-, $\gamma$-, $\delta$-, and $\zeta$-CAs. ${ }^{1-6}$ The $\alpha$ - $\beta$ - and $\delta$-CAs contain a $\mathrm{Zn}(\mathrm{II})$ ion at the active site, the $\gamma$-CAs are probably $\mathrm{Fe}(\mathrm{II})$ enzymes (but they are active also with $\mathrm{Zn}$ (II) or $\mathrm{Co}$ (II) ions), while the metal ion is usually replaced by cadmium in the $\zeta$-CAs. ${ }^{5,7-9}$

All these enzymes catalyze the reversible hydration of carbon dioxide to bicarbonate ion and proton $\left(\mathrm{CO}_{2}+\mathrm{H}_{2} \mathrm{O} \leftrightarrows \mathrm{HCO}_{3}^{-}\right.$ $+\mathrm{H}^{+}$), following a two step catalytic mechanism. ${ }^{2}$ In the hydration direction, the first step is the nucleophilic attack of a $\mathrm{Zn}^{2+}$-bound hydroxide ion on $\mathrm{CO}_{2}$ with consequent formation of $\mathrm{HCO}_{3}{ }^{-}$, which is then displaced from the active site by a water molecule (eq 1). The second step, which is rate limiting, regenerates the catalytically active $\mathrm{Zn}^{2+}$-bound hydroxide ion through a proton transfer reaction from the $\mathrm{Zn}^{2+}$-bound water molecule to an exogenous proton acceptor or to an active site residue, generically represented by B in eq 2 .

$$
\begin{aligned}
& \mathrm{EZn}^{2+}-\mathrm{OH}^{-}+\mathrm{CO}_{2} \\
& \quad \leftrightarrows \mathrm{EZn}^{2+}-\mathrm{HCO}_{3}^{-} \\
& \quad \leftrightarrows \mathrm{EZn}^{2+}-\mathrm{H}_{2} \mathrm{O}+\mathrm{HCO}_{3}^{-} \\
& \mathrm{EZn}^{2+}-\mathrm{H}_{2} \mathrm{O}+\mathrm{B} \leftrightarrows \mathrm{EZn}^{2+}-\mathrm{OH}^{-}+\mathrm{BH}^{+}
\end{aligned}
$$

All human CAs (hCAs) belong to the $\alpha$-class; up to now, fifteen isozymes have been identified, which differ by molecular features, oligomeric arrangement, cellular localization, distribution in organs and tissues, expression levels, kinetic properties and response to different classes of inhibitors (Table 1). ${ }^{2,10}$ Twelve isoforms (CA I-IV, VA-VB, VI-VII, IX, and XII$\mathrm{XIV}$ ) show a variable degree of enzymatic activity, whereas three isoforms (VIII, X, and XI), the so-called CA-related proteins (CARPs), are devoid of any catalytic activity (Table

Published: May 18, 2012 
Table 1. Organ/Tissue Distribution, Subcellular Localization, $\mathrm{CO}_{2}$ Hydrase Activity, and Affinity for Sulfonamides of the 15 Human $\alpha$-CA Isozymes ${ }^{2,10}$

\begin{tabular}{|c|c|c|c|c|}
\hline & organ/tissue distribution & subcellular localization & $\begin{array}{c}\text { catalytic activity }\left(\mathrm{CO}_{2}\right. \\
\text { hydration })\end{array}$ & $\begin{array}{l}\text { affinity for } \\
\text { sulfonamides }\end{array}$ \\
\hline CA I & erythrocytes, gastrointestinal tract, eye & cytosol & low & medium \\
\hline CA II & $\begin{array}{l}\text { erythrocytes, eye, gastrointestinal tract, bone osteoclasts, kidney, lung, } \\
\text { testis, brain }\end{array}$ & cytosol & high & very high \\
\hline CA III & skeletal muscle, adipocytes & cytosol & very low & very low \\
\hline CA IV & kidney, lung, pancreas, brain capillaries, colon, heart muscle, eye & membrane-bound & medium & high \\
\hline CA VA & liver & mitochondria & low & high \\
\hline CA VB & $\begin{array}{l}\text { heart and skeletal muscle, pancreas, kidney, spinal cord, gastrointestinal } \\
\text { tract }\end{array}$ & mitochondria & high & high \\
\hline CA VI & salivary and mammary glands & $\begin{array}{l}\text { secreted into saliva and } \\
\text { milk }\end{array}$ & low & very high \\
\hline CA VII & central nervous system & cytosol & high & very high \\
\hline CA VIII & central nervous system & cytosol & acatalytic & $\mathrm{ND}^{a}$ \\
\hline CA IX & tumours, gastrointestinal mucosa & transmembrane & high & high \\
\hline CA X & central nervous system & cytosol & acatalytic & $\mathrm{ND}^{a}$ \\
\hline CA XI & central nervous system & cytosol & acatalytic & $\mathrm{ND}^{a}$ \\
\hline CA XII & kidney, intestine, reproductive epithelia, eye, tumors & transmembrane & low & very high \\
\hline CA XIII & kidney, brain, lung, gut, reproductive tract & cytosol & low & high \\
\hline CA XIV & kidney, brain, liver, eye & transmembrane & low & high \\
\hline
\end{tabular}

1). ${ }^{2,5,11}$ Several studies demonstrated important roles of CAs in a variety of physiological processes, and showed that abnormal levels or activities of these enzymes have been often associated with different human diseases. ${ }^{2,3}$ Consequently, in recent years CA isozymes have become an interesting target for the design of inhibitors or activators with biomedical applications. ${ }^{2,12-18}$ CA activators may have pharmacological applications in pathologies in which learning and memory are impaired, such as Alzheimer's disease or aging. ${ }^{19}$ On the other hand, CA inhibitors (CAIs) have been originally used as diuretics, antiglaucoma agents, antiepileptics, and in the management of altitude sickness, ${ }^{2}$, while novel generation compounds are undergoing clinical investigation as antiobesity, and antitumor drugs/diagnostic tools. ${ }^{2,20-26}$

Table 2 shows a brief presentation of the various hCA isoforms as drug targets/off-targets. Indeed, some of them (such as CA I and II) are ubiquitous and may be both targets for some diseases and off-targets, and in this case their inhibition should be avoided (e.g., CA IX and XII in tumors should be inhibited by compounds, which do not affect the activity of CA I, II, VA, and VB). ${ }^{27}$ Specifically CA I is found in many tissues, but a seminal study from Feeener's group ${ }^{28}$ demonstrated that this enzyme is involved in retinal and cerebral edema, and its inhibition may be a valuable tool for fighting these conditions. CA II is involved in several diseases, such as glaucoma, edema, epilepsy, and probably altitude sickness. $^{26,29-33}$ CA III is involved in the oxidative stress, characterizing a lot of inflammatory diseases. It is not yet understood whether this feature is due to the $\mathrm{CO}_{2}$ hydration activity of CA III (which is quite low), or to other enzyme properties, such as a different enzymatic activity or the presence of Cys residues on its surface, responsible for the antioxidant effects of this protein. ${ }^{34-36}$ CA IV is surely a drug target for several pathologies, including glaucoma (together with CA II and XII), retinitis pigmentosa and stroke. ${ }^{37-40}$ The mitochondrial isoforms CA VA and VB are targets for obtaining antiobesity agents, ${ }^{20-22,41}$ whereas CA VI is implicated in cariogenesis. ${ }^{42,43} \mathrm{CA}$ VII has been noted for its contributions to epileptiform activity together with CA II and XIV. ${ }^{26,31,44}$ CA
Table 2. hCA Isoforms as Drug Targets/Offtargets in Various Diseases ${ }^{a}$

\begin{tabular}{|c|c|c|}
\hline isoform & $\begin{array}{l}\text { disease in which is } \\
\text { involved }\end{array}$ & $\begin{array}{l}\text { possible offtargets among other } \\
\text { hCAs }\end{array}$ \\
\hline CA I & retinal/cerebral edema ${ }^{28}$ & unknown \\
\hline \multirow[t]{4}{*}{ CA II } & glaucoma $^{29}$ & hCA I \\
\hline & edema $^{30}$ & unknown \\
\hline & epilepsy $^{26,31}$ & unknown \\
\hline & altitude sickness ${ }^{32,33}$ & unknown \\
\hline CA III & oxidative stress ${ }^{34-36}$ & unknown \\
\hline \multirow[t]{3}{*}{ CA IV } & glaucoma $^{37}$ & hCA I \\
\hline & retinitis pigmentosa ${ }^{38,39}$ & unknown \\
\hline & stroke $\mathrm{e}^{40}$ & unknown \\
\hline CA VA/VB & obesity $^{20-22,41}$ & hCA I, hCA II \\
\hline CA VI & cariogenesis $^{42,43}$ & hCA II \\
\hline CA VII & epilepsy ${ }^{26,31,44}$ & unknown \\
\hline \multirow[t]{2}{*}{ CA VIII } & neurodegeneration ${ }^{45}$ & unknown \\
\hline & cancer $^{45}$ & unknown \\
\hline CA IX & cancer $^{24,27,47}$ & hCA I, hCA II \\
\hline \multirow[t]{2}{*}{ CA XII } & cancer $^{24,47,48}$ & hCA I, hCA II \\
\hline & glaucoma $^{49}$ & unknown \\
\hline CA XIII & sterility $^{50}$ & unknown \\
\hline \multirow[t]{2}{*}{ CA XIV } & epilepsy $^{26,51}$ & unknown \\
\hline & retinopathy ${ }^{52}$ & unknown \\
\hline
\end{tabular}

${ }^{a}$ No data are available in the literature on CA X and XI involvement in diseases.

VIII was demonstrated to be involved in neurodegenerative diseases, as the CA 8 gene has been associated with ataxia, mild mental retardation and quadrupedal gait in humans and with lifelong gait disorder in mice, suggesting an important role for CA VIII in the brain. ${ }^{45}$ It was also found to be involved in the development of colorectal and lung cancers in humans, and its overexpression has been observed in several other cancers. ${ }^{45,46}$ However, nothing is known regarding the involvement of the remaining two acatalytic isoforms, CA X and XI, in human pathologies or what is the physiological function of these two proteins conserved all over the phylogenetic tree in vertebrates. ${ }^{45} \mathrm{CA}$ IX and XII are well-established anticancer 
drug targets. CA IX is a marker of disease progression in many types of hypoxic tumors, and recently its inhibition has been shown to be associated with a significant inhibition of the growth of both primary tumors and metastases. Furthermore, CAIs targeting this isoform can also be used for imaging of hypoxic tumors. ${ }^{24,27,47}$ CA XII is less investigated but basically it is also an antitumor target. ${ }^{24,47-49}$ There are a few reports regarding CA XIII showing that it is involved in the sperm motility processes ${ }^{50}$ (probably together with CA XIV) and the fact that its inhibition may be used in obtaining contraceptive agents. CA XIV is involved in epileptogenesis and, similarly to CA IV, in some retinopathies, and may be a drug target for innovative agents useful in the management of such disorders. $^{26,51,32}$

It should be stressed that none of the currently clinically used CAIs show selectivity for a specific isozyme., ${ }^{2,5}$ Thus, developing isozyme-specific CAIs should be highly beneficial in obtaining novel classes of drugs devoid of various undesired side-effects. Recently, a large number of structural studies has provided a scientific basis for the rational drug design of more selective enzyme inhibitors. ${ }^{53}$ However, although X-ray crystal structures are already available for the majority of the twelve catalytically active members of the human CA family, ${ }^{54-65}$ most of the reported complexes with inhibitors regards just isozyme II, the most thoroughly characterized CA isoform.

The aim of this review is to provide an exhaustive description of the structural studies on $\alpha$-CAs so far reported. In particular, the main structural features of the catalytically active $\alpha$-CA isozymes characterized to date will be described and the current state of the art on complexes of hCA II with the principal classes of inhibitors will be summarized. A comparison with the corresponding adducts of other isoforms, when available, will be also performed, in order to find for each isoform the key residues responsible for enzyme/inhibitor interaction. Finally, recent advances in the field of drug design of selective CAIs will be illustrated.

\section{STRUCTURAL FEATURES OF $\alpha$-CAs}

As mentioned above, human catalytically active $\alpha$-CAs differ for subcellular localizaton: CA I, II, III, VII and XIII exist in cytosol, CA IV, IX, XII, and XIV are membrane-associated, CA $\mathrm{VA}$ and VB reside in mitochondria, whereas CA VI is secreted in saliva and milk (Figure 1). ${ }^{2,5}$ To date the three-dimensional structures of all isoforms except CA VB have been determined. $^{54-65}$ The analysis of these structures shows that independently on their subcellular localization and, as expected on the basis of their high sequence homology (Figure 2), these enzymes present a similar structure, characterized by a central twisted $\beta$-sheet surrounded by helical connections and additional $\beta$-strands (Figure 3 ). The active site is located in a large, conical cavity, approximately wide $12 \AA$ and deep $13 \AA$, which spans from the protein surface to the center of the molecule. The catalytic zinc ion is located at the bottom of this cavity, exhibiting a tetrahedral coordination with three conserved His residues and a water molecule/hydroxide ion as ligands (Figure 3). ${ }^{11,66-68}$ The $\mathrm{Zn}^{2+}$-bound water molecule/ hydroxide ion is involved in a network of hydrogen bonds which helps to enhance its nucleophilicity. In particular, it is hydrogen bonded with the hydroxyl moiety of a conserved Thr residue (Thr199) and with two water molecules, located on two opposite sides: the first one, also called the "deep water", is located in a hydrophobic cavity delimited by conserved residues in position 121, 143,198, and 209, while the second one is in a

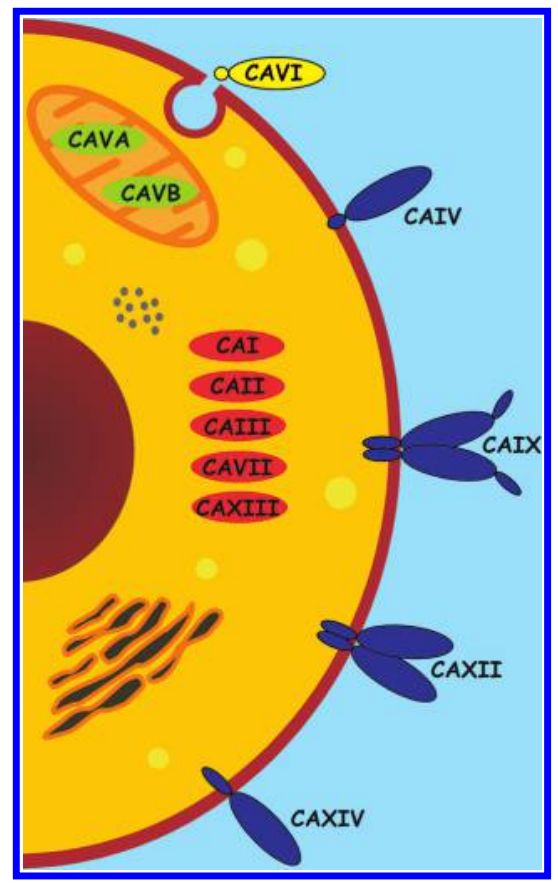

Figure 1. Schematic illustration of domain composition and subcellular localization of catalytically active human $\alpha$-CAs: the cytosolic CA I, II, III, VII, and XIII and the mitochondrial CA VA and $\mathrm{VB}$ consist only of the CA domain; the membrane-associated CA IV, IX, XII, and XIV have a transmembrane anchor and, except for CA IV, also a cytoplasmic tail, while CA IX is the only isozyme with an $\mathrm{N}$ terminal proteoglycan-like domain; CA VI is secreted and contains a short C-terminal extension.

hydrophilic environment toward the entrance of the active site (Figure 4). ${ }^{69}$ In all the CA isoforms the active site cavity is divided into two very different environments: the first one is delimited by a cluster of hydrophobic amino acids, whereas the other one is lined with hydrophilic residues (Figure 5). Several studies suggested that these two peculiar active site environments are responsible of the rapid catalytic cycling of $\mathrm{CO}_{2}$ to bicarbonate; ${ }^{70}$ indeed, the hydrophobic region is necessary to sequester the $\mathrm{CO}_{2}$ substrate and orient the carbon atom for nucleophilic attack by the zinc-bound hydroxide, ${ }^{70,71}$ while the hydrophilic region creates a well ordered hydrogen-bonded solvent network, which is necessary to allow the proton transfer reaction from the zinc-bound water molecule to the bulk solvent (eq 2 and Figure 4). ${ }^{70,72-75}$ Despite the high sequence and structural homology among all the isozymes, the oligomeric state represents a discriminating factor at least for three of them. Indeed, all the enzymes are monomeric, except the two membrane-associated isoforms CA IX and CA XII and the secreted CA VI, which in contrast are dimeric. ${ }^{63,65}$ Intriguingly, the dimeric structures of these three isoforms are different, and none influences the catalytic properties of the enzyme. The observation of a dimeric arrangement in only three $\alpha$-CAs is quite surprising and probably suggests that the oligomeric arrangement of these isoforms responds to the necessity to perform specific functions in the tissues, where they are generally present. ${ }^{59,63}$

\section{INSIGHTS INTO CA CATALYTIC MECHANISM: $\mathrm{CO}_{2}$ AND $\mathrm{HCO}_{3}{ }^{-}$BINDING TO hCA II}

Even if in the past a high number of studies provided hypotheses on the putative binding site of $\mathrm{CO}_{2}$ in the CA active 


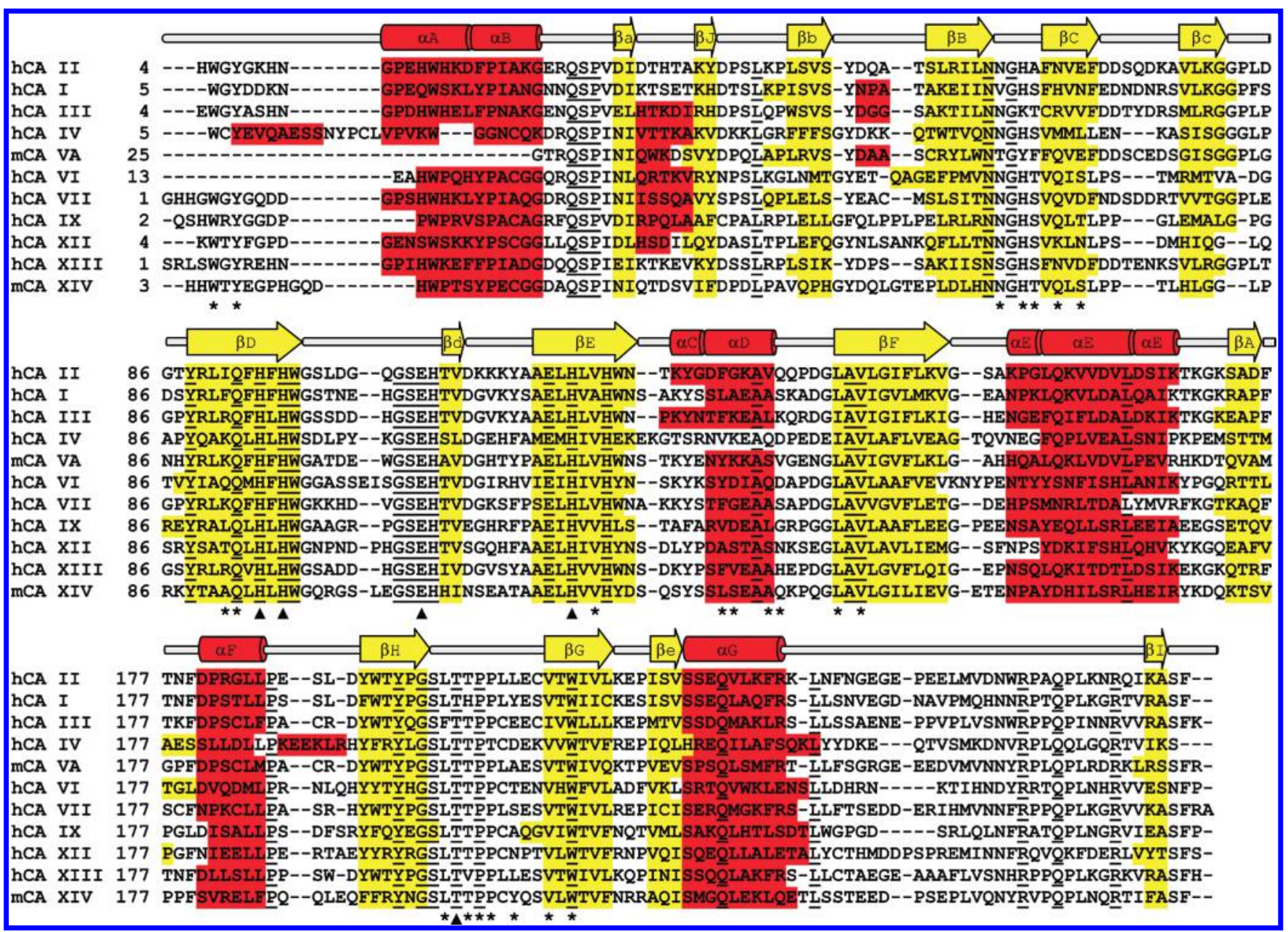

Figure 2. Structure-based sequence alignment of $\alpha$-CAs with known three-dimensional structure. hCA II secondary structure elements are shown schematically: helices are represented by solid cylinders and $\beta$-strands as arrows. Helix and $\beta$-strand regions for the various isozymes are highlighted in red and yellow, respectively. Conserved residues are underlined, catalytic triad, Thr199 and Glu106 are indicated with black triangles, while residues delimiting the active site cavity are marked with asterisks. $\beta$-strand and helix regions are named as reported by Eriksson et al. ${ }^{55}$ The following PDB entries were used in the alignment: 1CA2 (hCA II); ${ }^{55}$ CAB (hCA I); ${ }^{54} 1 \mathrm{Z93}$ (hCA III); ${ }^{61}$ 1ZNC (hCA IV); ${ }^{58}$ 1DMX (mCA VA); $;^{57}$ 3FE4 (hCA VI); ${ }^{65}$ 3ML5 (hCA VII); ${ }^{64}$ 3IAI (hCA IX); ${ }^{63} 1 \mathrm{JCZ}$ (hCA XII); ${ }^{59}$ 3D0N (hCA XIII); ${ }^{62}$ 1RJ5 (mCA XIV). ${ }^{60}$

site, ${ }^{71,76-81}$ the first clear structural evidence on the $\mathrm{CO}_{2}$ binding region was obtained only recently with two independent crystallographic studies showing the entrapment of this substrate in the CA II active site. ${ }^{69,70}$ Figure $6 \mathrm{~A}$ shows the binding interactions of $\mathrm{CO}_{2}$ as determined by these structural studies. In particular, the $\mathrm{CO}_{2}$ molecule is bound in the hydrophobic side of the active site, in the small pocket delimited by residues Val121, Val143, Leu198 and Trp209 and in an orientation that makes it suited for the nucleophilic attack on the carbon atom by the zinc bound hydroxide ion. One of the $\mathrm{CO}_{2}$ oxygen atoms lies about in the same position formerly occupied by the "deep water", which is now shifted in a new position, where it establishes novel hydrogen bond interactions (see Figure 4 and Figure 6A). These structural data are in agreement with previously reported mutagenesis studies which demonstrated that substitutions of Val121, Val143, and Leu198 with bulkier residues, leading to the reduction of the volume of the $\mathrm{CO}_{2}$ binding cavity, caused significant decreases in the activity. ${ }^{80-83}$ Interestingly, the presence of the $\mathrm{Zn}^{2+}$ ion seems to be not important in the binding and orientation of $\mathrm{CO}_{2}{ }^{70,84}$ The structure of hCA II/ $\mathrm{HCO}_{3}{ }^{-}$complex was also recently provided by Sjoblom and co-workers. ${ }^{69}$ As shown in Figure 6B the $\mathrm{HCO}_{3}{ }^{-}$lies in the same plane defined by the $\mathrm{CO}_{2}$ molecule
(Figure 6A) and the $\mathrm{Zn}^{2+}$-bound hydroxide ion and is tetrahedrally coordinated to the catalytic metal ion through one of its oxygen atoms. The availability of both hCA II/ $\mathrm{CO}_{2}$ and hCA II $/ \mathrm{HCO}_{3}{ }^{-}$complex structures allowed to obtain for the first time a detailed description of the first step of the enzymatic reaction providing new perspectives in the structural studies of this exciting enzyme family.

\section{INSIGHTS INTO CA INHIBITION: STRUCTURAL FEATURES OF ZINC BINDING INHIBITORS}

Analysis of all available structural studies for $\mathrm{CA} /$ inhibitor complexes allowed to divide CA inhibitors into two main classes: those that bind to the enzyme active site anchoring themselves to the catalytic zinc ion, and those that are bound to the active site but do not interact directly with the metal ion. Four different groups of zinc binding inhibitors have been studied crystallographically: the ureates/hydroxamates, the mercaptophenols, the metal-complexing anions and the sulfonamides with their bioisosteres, such as sulfamates and sulfamides. ${ }^{53}$ In this chapter the main structural features of the binding of these molecules to the CA II active site will be schematically summarized. 


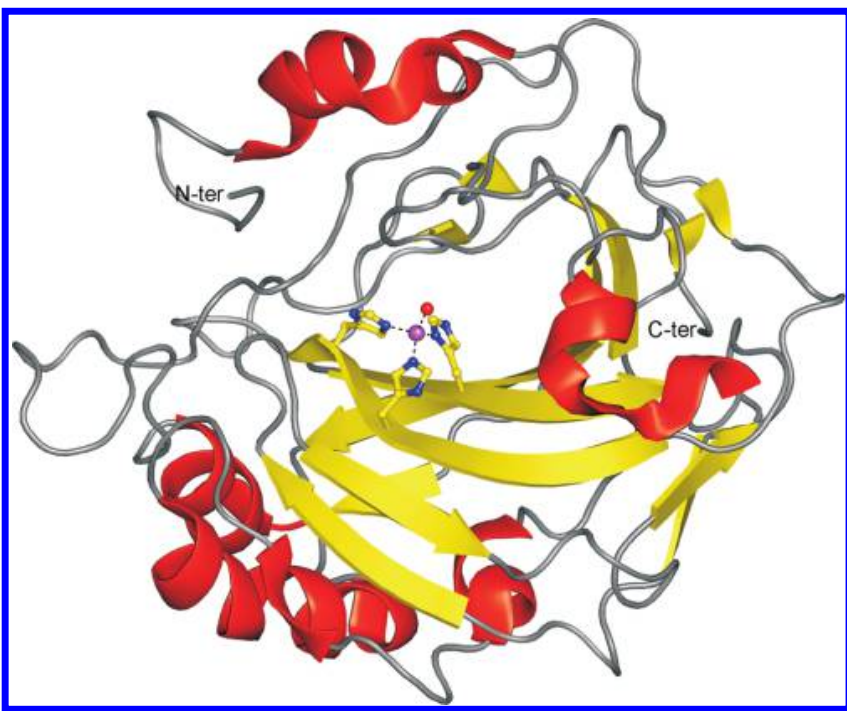

Figure 3. Ribbon diagram of hCA II structure (PDB code 1CA2), which has been chosen as representative CA isoform. The active site $\mathrm{Zn}^{2+}$ coordination is also shown. Helix and $\beta$-strand regions are colored in red and yellow, respectively.

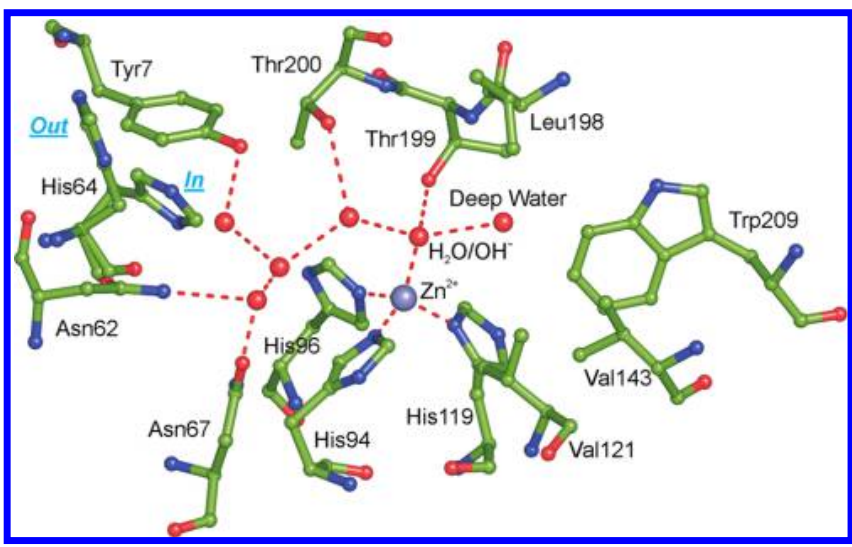

Figure 4. The active site of hCA II, which has been chosen as representative CA isoform. The $\mathrm{Zn}^{2+}$ is tetrahedrally coordinated by the three catalytic histidines and a water molecule/hydroxide ion, which is engaged in a well-defined network of hydrogen bonds. Water molecules are indicated as red circles. The side chain of His64 is shown in both the in and out conformations.

\subsection{Binding of Ureates and Hydroxamates}

hCA II is not only a very effective catalyst for the interconversion between carbon dioxide and bicarbonate, but shows some catalytic versatility, participating in several other hydrolytic processes which presumably involve nonphysiological substrates (see Scheme 1). These reactions include the hydration of cyanate to carbamic acid (eq 3), ${ }^{85}$ or of cyanamide to urea (eq 4), ${ }^{86,87}$ the aldehyde hydration to gem-diols (eq $5),{ }^{88,89}$ the hydrolysis of some carboxylic (eq 6) ${ }^{90-92}$ or sulfonic acid esters (eq 7), ${ }^{92}$ as well as other less investigated hydrolytic processes (eq 8-11). Among these reactions, the hydration of cyanamide to urea has been carefully characterized by means of spectroscopic, kinetic, and crystallographic techniques. ${ }^{86,87}$ These studies demonstrated that cyanamide acts as a weak inhibitor of the esterase activity of hCA II, interacting with the zinc ion within the enzyme active site. Interestingly, when hCA II crystals were soaked with cyanamide for 90 min two different adducts with different occupancy were

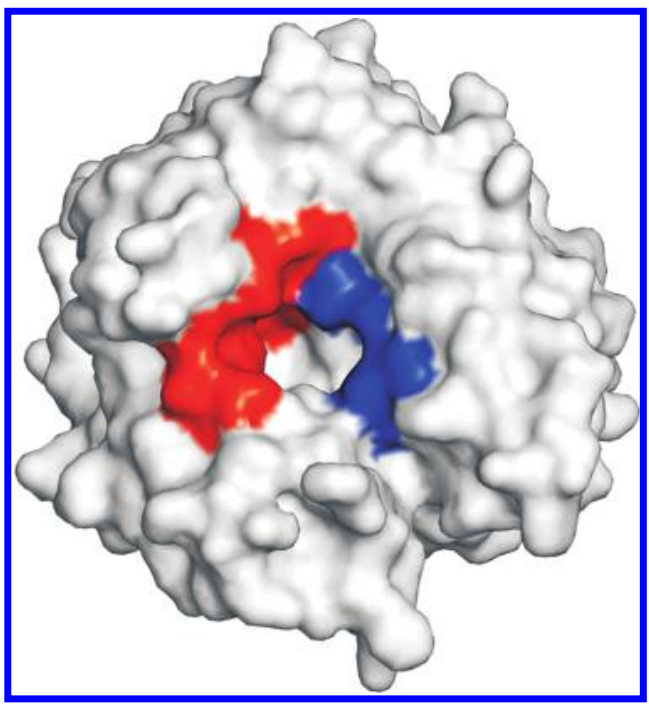

Figure 5. Solvent accessible surface of hCA II. Residues delimiting the hydrophobic half of the active site cleft are shown in red (Ile91, Phe131, Val121, Val135, Leu141, Val143, Leu198, Pro202, Leu204 Val207 and Trp209), while residues delimiting the hydrophilic one are shown in blue (Asn62, His64, Asn67 and Gln92).

observed in the active site. In the high occupancy form the cyanamide replaces the hydroxide ion coordinating to the zinc ion with a regular tetrahedral geometry. In the low occupancy

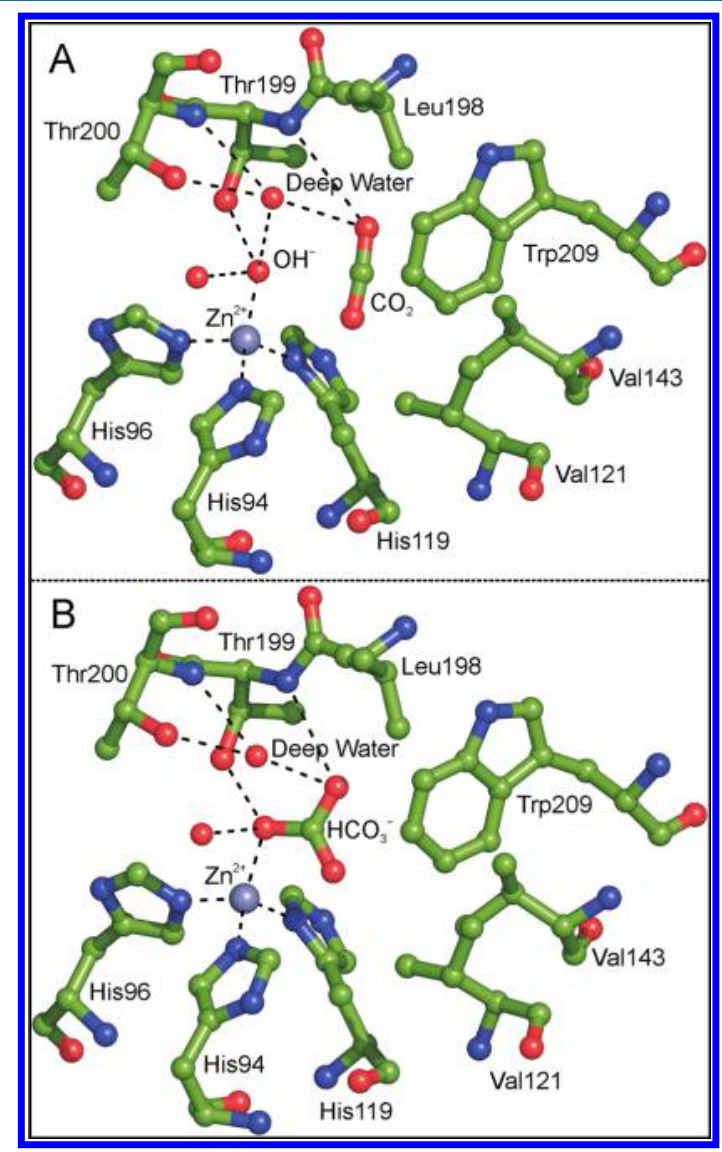

Figure 6. Active site of hCA II showing: (A) the position of $\mathrm{CO}_{2}$ molecule (PDB code 2VVA), (B) the binding of the bicarbonate ion (PDB code $2 \mathrm{VVB})$. The $\mathrm{Zn}^{2+}$ coordination and polar interactions are also reported. 
Scheme 1. Examples of Known Reactions Catalyzed by hCA II

\begin{tabular}{|l|}
\hline $\mathrm{HNCO}+\mathrm{H}_{2} \mathrm{O} \leftrightarrows \mathrm{H}_{2} \mathrm{NCOOH}$ \\
$\mathrm{HNCNH}+\mathrm{H}_{2} \mathrm{O} \leftrightarrows \mathrm{H}_{2} \mathrm{NCONH}_{2}$
\end{tabular}

form cyanamide adds to the coordination sphere ${ }^{87}$ generating a pentacoordinated adduct together with a zinc-bound water molecule, which can be considered as a zinc outer-sphere fifth ligand (Figure 7A). While the first adduct was considered a

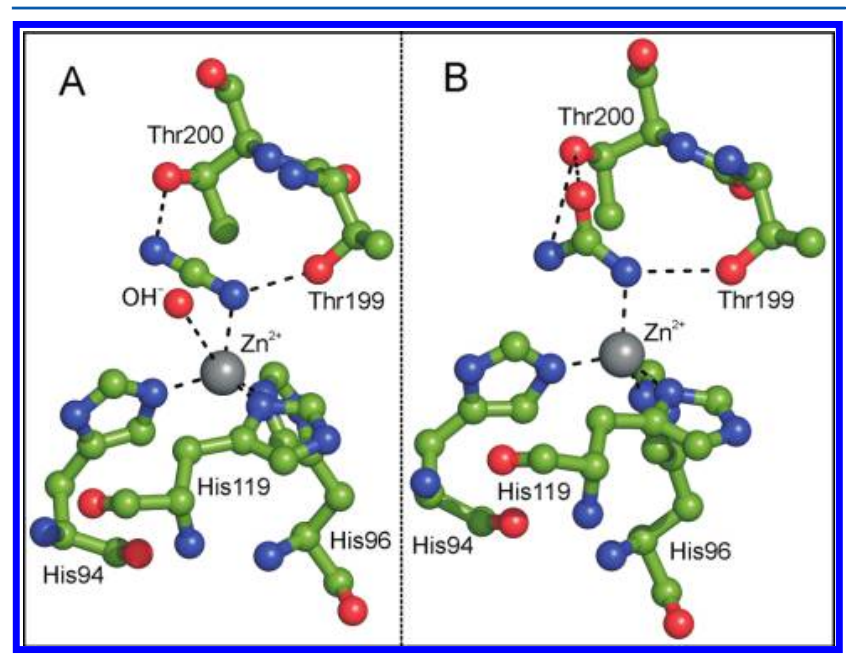

Figure 7. Representation of the binding mode of (A) cyanamide (PDB code $1 \mathrm{~F} 2 \mathrm{~W}$ ) (pentacoordinated adduct), and (B) urea (PDB code 1BV3) to the hCA II active site.

dead-end product, the second one represented an intermediate of the catalyzed reaction, where the hydroxide ion was ready to perform the nucleophilic attack on the substrate cyanamide forming urea. In agreement with this hypothesis, when the hCA II crystals were soaked in cyanamide solutions for several hours, the reaction took place with the resulting urea which remained bound to the metal ion (Figure 7B) ${ }^{86}$ The observation of a strong binding between urea and the zinc ion has opened the way to new studies on the interactions of CAs with small molecules possessing appropriate structural features to act as zinc binding functions. Among these molecules, $N$-hydroxyurea represents one of the simplest compounds. ${ }^{93}$ The crystallographic structure of the hCA II/N-hydroxyurea adduct showed that this molecule binds to the $\mathrm{Zn}^{2+}$ ion of hCA II active site in a bidentate mode, by means of the oxygen and nitrogen atoms of the $\mathrm{NHOH}$ moiety. Additional hydrogen bonds involving the hydroxyl and the carbonyl moieties of the inhibitor and the enzyme residue Thr199 were also observed (Figure 8A). ${ }^{94}$ Worth noting is that the related acetohydroxamic and

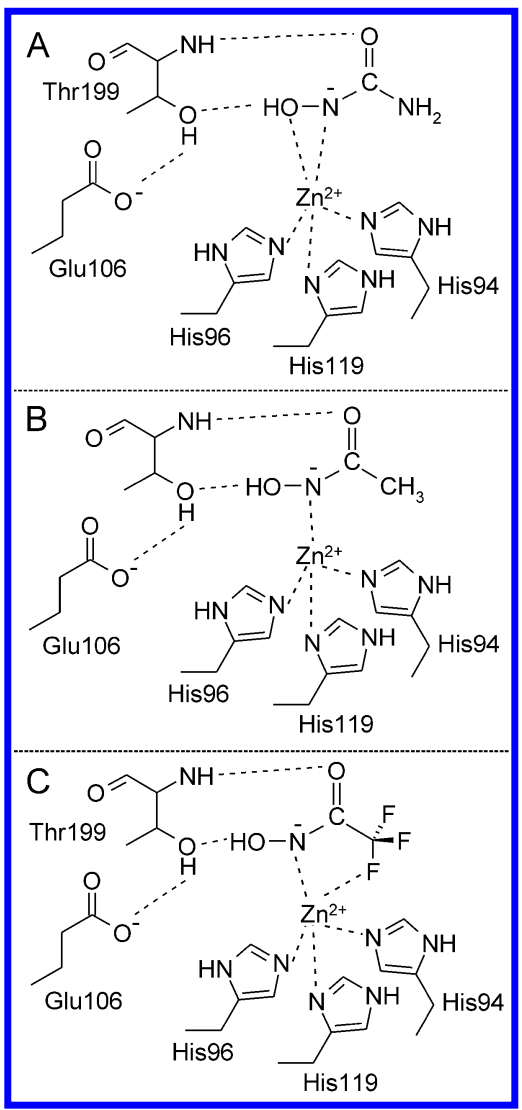

Figure 8. Schematic representation of (A) N-hydroxyurea (PDB code 2GEH), (B) acetohydroxamic acid (PDB code 1AM6), and (C) trifluoroacetohydroxamic acid to the hCA II active site.

trifluoroacetohydroxamic acids, although containing the same hydroxamate functionality, were demonstrated to adopt a very different binding mode. In particular, in the case of acetohydroxamic acid, the ionized nitrogen was directly bound to the $\mathrm{Zn}^{2+}$ ion in a tetrahedral coordination (Figure $8 \mathrm{~B}$ ), while in the case of trifluoroacetohydroxamic acid a fluorine atom and the ionized nitrogen formed a 5-membered chelate with zinc ion, with a coordination geometry being approximately trigonal bipyramidal (Figure 8C). ${ }^{95}$ Altogether these findings suggest that the hydroxamate moiety represents a very versatile zinc binding function which can adopt different binding modes to the metal ion present in the CA active site. Therefore it could be usefully exploited for obtaining potent CA inhibitors.

\subsection{Thiol Derivatives}

Thioxolone (1) is a biologically active compound, classified as an antiseborrheic agent ${ }^{96}$ largely used in the treatment of acne and dandruff. ${ }^{97}$ Cytostatic, ${ }^{98}$ antipsoriatic, antibacterial, and antimycotic activities ${ }^{96}$ were also reported as medically useful properties of this molecule. A screening study on a series of biologically active compounds against hCA II led to identify this molecule as a weak inhibitor for this enzyme. ${ }^{99}$ Since $\mathbf{1}$ does not contain any functional group typically found in therapeutic CA inhibitors, several biochemical and structural studies were devoted to understand its inhibition mechanism. A preliminary characterization revealed that $\mathbf{1}$ acts as a weak hCA II inhibitor, both in a kinetic esterase assay with the substrate 4nitrophenyl acetate (4-NPA) and in a competitive binding assay with dansylamide $\left(\mathrm{IC}_{50}\right.$ value of $1.8 \mu \mathrm{M}$ and $K_{\mathrm{d}}$ value of 33 
$\mu \mathrm{M}) .{ }^{100}$ The inhibition mechanism of this molecule was not immediately clear analyzing its chemical structure but crystallographic studies helped to clarify it. ${ }^{100}$ In particular, in hCA II crystals soaked with $\mathbf{1}$, the latter was not found in the active site, which instead contained a 4-mercaptobenzene-1,3-diol molecule (2) bound to the catalytic zinc ion (Figure 9). This

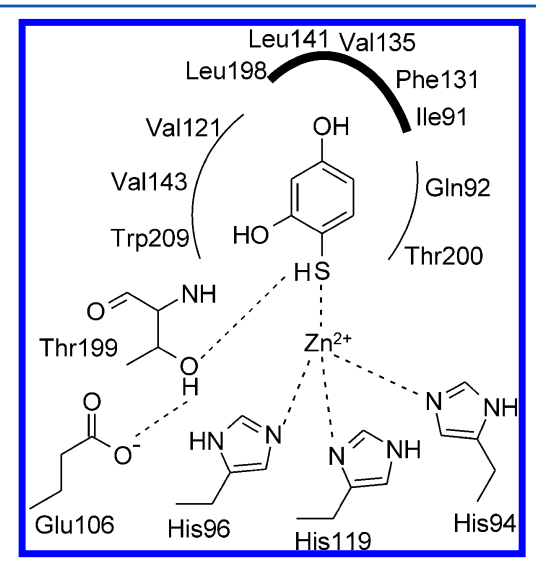

Figure 9. Schematic representation of the active site region in the hCA II/2 complex (PDB code 2OSF). Residues involved in inhibitor recognition are shown. The hydrophobic pocket which accommodates the hydroxyl group in para-position to the thiol moiety is highlighted in bold.

observation led to hypothesize that when thioxolone binds to the hCA II active site, it is cleaved forming 4-mercaptobenzene1,3-diol via the intermediate $S$-(2,4-thiophenyl)hydrogen thiocarbonate (see Scheme 2) utilizing, as hypothesized for

\section{Scheme 2. Thioxolone Hydrolysis Catalyzed by hCA II}

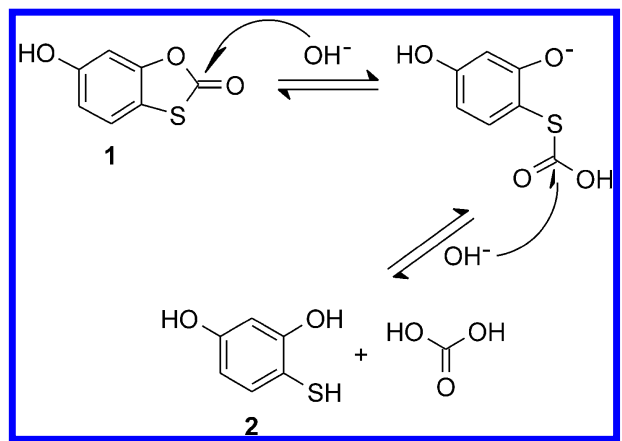

other esters, ${ }^{101}$ a hCA II zinc-hydroxide mechanism. ${ }^{100}$ The main stabilizing interactions observed in the so-formed hCA II/ 2 adduct are schematically depicted in Figure 9. The inhibitor is coordinated to the zinc ion through its thiol group, which also forms a weak hydrogen-bond with Thr199OG1 atom, similarly to the interaction previously observed in several sulfonamide/ sulfamide/sulfamate complexes. ${ }^{53}$ The phenyl ring is stabilized by several van der Waals interactions with active site residues, while the hydroxyl group in para-position to the thiol moiety points toward a hydrophobic pocket delimited by residues Ile91, Phe131, Val135, Leu141, and Leu198 and establishes a series of nonfavorable interactions. These findings prompted the authors to investigate also some thioxolone structural analogues as hCA II inhibitors, such as the thioxolone hydrolysis product 2 , and the two smaller related compounds 2-hydroxythiophenol (3) and benzenethiol (4). The latter two

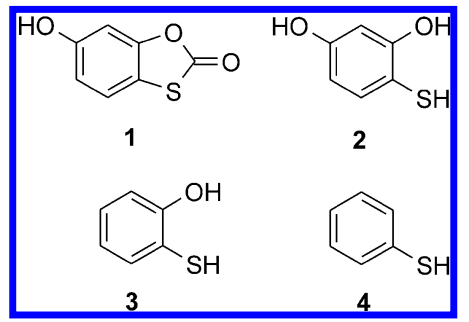

compounds behaved as hCA II inhibitors in a 4-NPA esterase assay with $\mathrm{IC}_{50}$ values of 1.05 and $1.49 \mu \mathrm{M}$, thus appearing more potent inhibitors than the parent compound, thioxolone. Inhibition constants of $\mathbf{1}, \mathbf{2}$, and $\mathbf{3}$ were also determined for the physiologically relevant reaction of $\mathrm{CO}_{2}$ hydration. In this case $\mathrm{K}_{\mathrm{I}}$ values of 314,148 , and $0.631 \mu \mathrm{M}$ for 1,2 , and 3 , respectively were determined. These results further confirmed that thioxolone is a relatively weak inhibitor of hCA II activity, whereas its hydrolysis product 2 , although still a relatively weak inhibitor, is twice more potent. Again, 3 shows a significant 500 -fold increase in inhibition, similar in magnitude to other sulfonamide inhibitors. This result suggested that the absence of the hydroxyl group in para-position to the thiol moiety of $\mathbf{2}$ may have greatly improved the affinity of this molecule toward the CA active site, in agreement with structural studies described above, which highlighted the incompatibility of the hydrophilic nature of this group in the hydrophobic pocket.

In conclusion, these studies demonstrate that mercaptophenols represent a class of compounds with good CA inhibitory properties, ${ }^{100,102}$ even if they lack functional groups typically found in therapeutically used CA inhibitors.

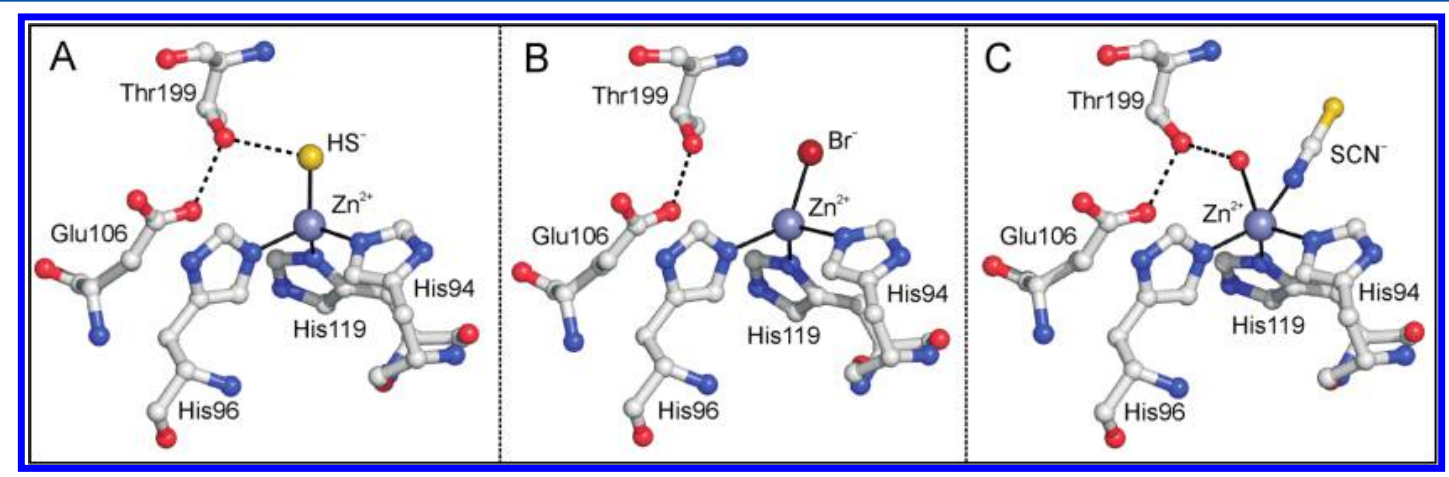

Figure 10. Representation of the three different $\mathrm{Zn}^{2+}$ coordination geometries of some representative anions: (A) hydrogen sulfide (PDB code 1CAO), (B) bromide (PDB code 1RAZ), and (C) thiocyanate (PDB code 2CA2). Hydrogen bonds are represented as dotted lines, whereas zinc ion coordination as solid lines. 


\subsection{Metal-Complexing Anions}

Inorganic anions constitute a well-known class of CAIs. They are usually weak inhibitors with affinities in the millimolarsubmillimolar range, with a few anions arriving to be low micromolar inhibitors for some isoforms (e.g., cyanide, azide, hydrogen sulfide and trithiocarbonate). $2,11,53,103$

Crystallographic studies have shown that inorganic anions can bind within the CA II active site, either coordinating the catalytic $\mathrm{Zn}^{2+}$ ion or not coordinating it, but being located in its close proximity in a nonmetal site. ${ }^{2,11,53,104,105}$ When these anions coordinate the $\mathrm{Zn}^{2+}$ ion, three different coordination geometries have been observed: regular tetrahedral, ${ }^{66,105}$ distorted tetrahedral ${ }^{103,106,107}$ and trigonal bipyramid. ${ }^{55,66}$ This different behavior can be ascribed to the structural features of the ligands. In particular, anions able to act as hydrogen-bonding donor toward the active site residue Thr199, such as hydrogen sulfide $\left(\mathrm{HS}^{-}\right)^{105}$ and hydrogen bisulfite $\left(\mathrm{HSO}_{3}{ }^{-}\right),{ }^{66}$ replace the zinc-bound water/hydroxide adopting a regular tetrahedral coordination geometry (Figure 10A). ${ }^{53,66,105,108}$ Unprotonated ligands can adopt either distorted tetrahedral or trigonal bipyramid coordination geometry. In particular, anions as bromide $\left(\mathrm{Br}^{-}\right),{ }^{106}$ and azide $\left(\mathrm{N}_{3}^{-}\right)^{106,107}$ still coordinate to the zinc ion replacing the zinc bound water/hydroxide. However, since these inhibitors cannot form a hydrogen bond with Thr1990G1, the coordination around $\mathrm{Zn}^{2+}$ is significantly distorted from a regular tetrahedral geometry (Figure 10B). ${ }^{106,107}$ Finally, anions as formate ${ }^{66}$ and thiocyanate ${ }^{109}$ bind to the enzyme active site by addition to the metal coordination sphere, shifting the zincbound solvent molecule and generating a distorted trigonal bipyramid geometry (Figure 10C). ${ }^{6,109}$

\subsection{Sulfonamides}

To date, the sulfonamide group $\left(\mathrm{R}-\mathrm{SO}_{2} \mathrm{NH}_{2}\right)$ is the most important and largely used zinc binding function for the design of CAIs; accordingly, the majority of the clinically used CAIs are sulfonamides. Since the first evidence of their CA inhibitory properties, ${ }^{110}$ these molecules were largely investigated by means of kinetic, physiological and pharmacological studies. $^{2,5,10,25,53,111}$ Crystallographic studies are also available for many of their adducts with several CA isozymes, ${ }^{53,63,64}$ elucidating the key factors responsible for the binding of the sulfonamide moiety to the CA active site and providing a rationale for the unique tailored properties of this anchoring group. In all the studied adducts the binding of the sulfonamide derivatives is mainly driven by the coordination of the deprotonated sulfonamide nitrogen to the catalytic $\mathrm{Zn}^{2+}$ ion, ${ }^{112}$ with consequent substitution of the zinc-bound water molecule, and by two $\mathrm{H}$-bonds of the sulfonamide moiety with residue Thr199 (Figure 11). ${ }^{13,67,108,113}$ Furthermore, depending on the nature of the $\mathrm{R}$ substituent, additional interactions with the hydrophilic and/or hydrophobic region of the active site can be also present (Figure 11). ${ }^{53}$ These studies highlighted that the sulfonamide group is an ideal ligand of the CA active site for two main reasons: on one hand, it combines the negative charge of the deprotonated nitrogen with the positively charged zinc ion, on the other hand, the presence of one proton on the coordinated nitrogen atom satisfies the hydrogen bond acceptor character of Thr1990G1 atom, which forms a strong H-bond with it. ${ }^{5,53}$

4.4.1. Benzenesulfonamides. The first evidence that sulfonamides could act as potent CAIs came from a study, reported by Mann and Keilin in $1940,{ }^{110}$ on sulfanilamide 5 .

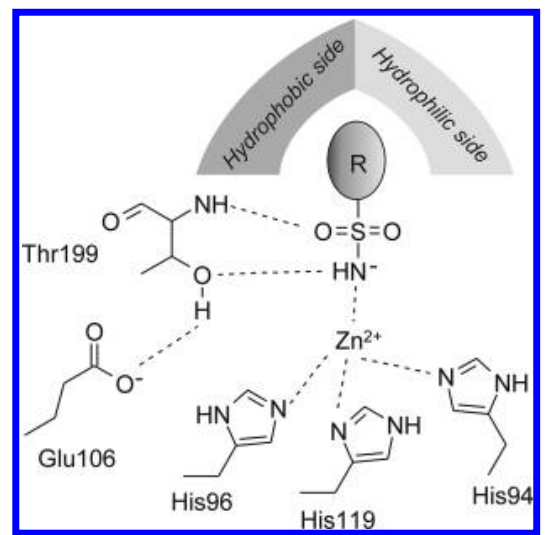

Figure 11. Schematic illustration of the key interactions between a generic sulfonamide inhibitor and the hCA II active site.

Subsequently, many aromatic sulfonamides were synthesized and investigated for their CA inhibitory action. ${ }^{114}$ Among these, benzenesulfonamides, of which 6 is the prototype, constitute the most common and best characterized class. To date, a very large number of studies has been reported on the interaction of these molecules with CA II active site, which allowed to obtain the following structure-activity relationships: (i) 2-substituted, 2,4-, and 3,4-disubstituted benzenesulfonamides behave generally as weaker inhibitors compared with 4-substituted derivatives; (ii) 4-substituents inducing good activities include halogens, acetamido and alkoxycarbonyl moieties as well as esters of 4-sulfamoylbenzoic acid; (iii) good inhibitory properties and interesting physicochemical features were observed for compounds possessing carboxy-, hydrazido-, ureido-, thioureido-, and methylamino-moieties in position $4^{53,114-116}$ Several structural studies on the adducts that benzensulfonamides form with hCA II have also been published and have provided an unambiguous view of the molecular determinants responsible for the enzyme-inhibitor interactions. 5,11,25,53,104 Interestingly, in nearly all the studied adducts, the interaction of the benzenesulfonamide moiety with the enzyme active site is rather similar, with the sulfonamide moiety involved in the canonical coordination of the $\mathrm{Zn}^{2+}$ catalytic ion (see above) and the phenyl ring, which establishes several van der Waals interactions with residues Gln92, Val121, Phe131, Leu198, and Thr200 (Figure 12). On the contrary, depending on their nature and position, the substituents of the phenyl ring

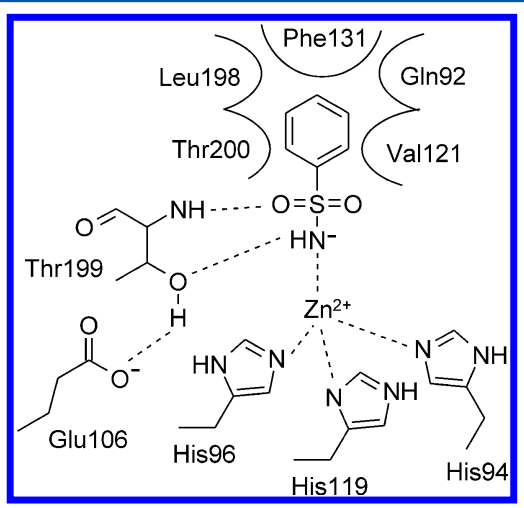

Figure 12. Schematic representation of the binding mode of the benzenesulfonamide 6 with the hCA II active site (PDB code 2WEJ). Residues establishing van der Waals interactions (distance $<4.5 \AA$ ) with the inhibitor phenyl ring are also shown. 
can establish different types of interactions which involve the hydrophobic and/or the hydrophilic region of the active site. In the following paragraphs the numerous CAIs belonging to the benzenesulfonamide class will be reviewed, highlighting the main structural determinants responsible of their hCA II inhibitory properties. To simplify the discussion, these molecules will be grouped on the basis of either the functional groups used to derivatize the phenyl ring, or their biological action.<smiles>Nc1ccc(S(N)(=O)=O)cc1</smiles>

5

4.4.1.1. Halogenated Benzenesulfonamides. Introduction of halogen atoms as substituents in various positions of the benzene ring of benzenesulfonamides has been largely investigated from diverse points of view. Several studies suggested that the presence of such electronegative substituents exerts an acidifying effect on the sulfonamide protons, which is thought to be correlated with an increase in the CA inhibitory properties. ${ }^{5,53,117}$ Consequently, although this seems not to be always true, ${ }^{117}$ the presence of one or more halogen atoms in the scaffold of a benzenesulfonamide CAI is usually correlated with efficient inhibition of many isoforms, such as CA I, II, VII, IX, XII, etc. ${ }^{117,118}$ A detailed study on the way in which chlorine and/or fluorine atoms present in the benzenesulfonamide scaffold influence the binding mode to the enzyme and consequently the thermodynamics of binding has been recently reported by Scott and co-workers. ${ }^{119}$ In this study, the crystallographic structures of benzenesulfonamide $\mathbf{6}$ and its chloro-/fluoro-substituted derivatives 7-9 in complex with hCA II were reported and correlated with an isothermal titration calorimetry (ITC) analysis, highlighting that often very similar substituents (e.g., $\mathrm{F}$ or $\mathrm{Cl}$ in position 2 or 3 ) can cause important differences on the binding mode to the enzyme (see Figure 13) and consequently significant changes in the thermodynamics of binding. ${ }^{119}$

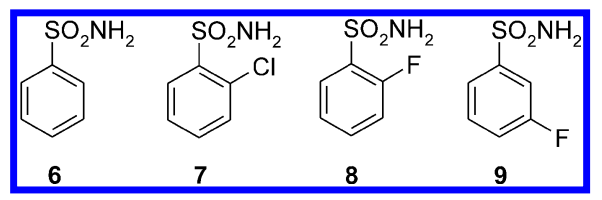

These findings showed the difficulties in the prediction of the effect of a substitution on the phenyl ring of the benzenesulfonamide scaffold and highlighted the helpfulness of a combined thermodynamic and structural approach in the rational drug design of new CAIs.

4.4.1.2. Sulfamoyl-Benzenecarboxamides. Sulfamoyl-benzenecarboxyamides of type $\mathbf{1 0}$ and $\mathbf{1 1}$ represent an important class of CAIs obtained by reaction of 4-carboxy-benzenesulfonamide (12) or 4-chloro-3-sulfamoyl benzoic acid (13) with different molecules containing primary amino groups. ${ }^{120-132}$ The nature of the $\mathrm{R}$ group can be very different: it can vary from a peptide tail to an aliphatic spacer, and depending on its features the corresponding inhibitors present different affinity toward various CA isozymes as well as diverse physicochemical properties.

Initial studies on this class of derivatives were performed by Whitesides and Christianson groups, which investigated the

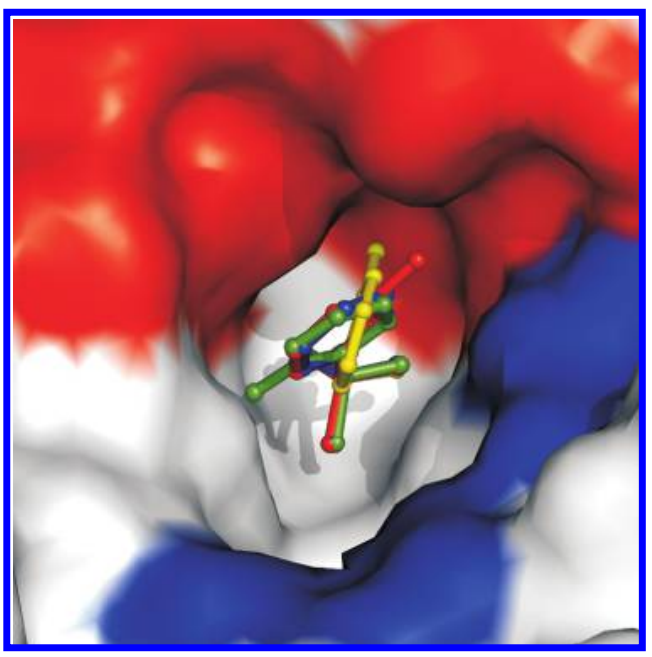

Figure 13. Superposition of hCA II/inhibitor adducts: 6 is reported in blue (PDB code $2 \mathrm{WEJ}$ ), 7 in yellow (PDB code $2 \mathrm{WEH}$ ), 8 in green (PDB code 2WEG) and 9 in red (PDB code 2WEO). This picture shows as the aromatic ring orientation is conserved for 6,8 , and 9 , whereas the phenyl moiety is twisted in the case of 7 , allowing the chlorine substituent to better interact with the hydrophobic side of the hCA II active site. The enzyme is represented as a surface-model (hydrophobic side in red, hydrophilic side in blue).

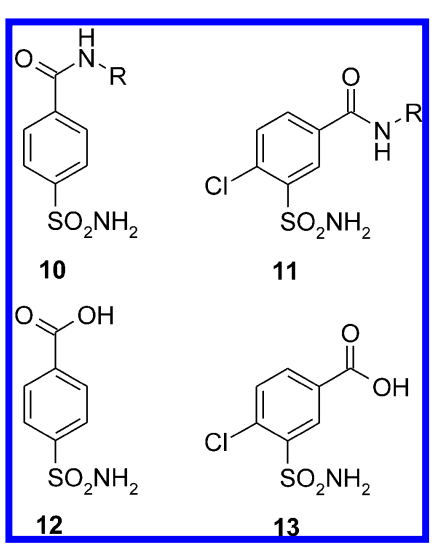

derivatization of $\mathbf{1 2}$ with oligopeptide tails, generating compounds of type $\mathbf{1 4}, \mathbf{1 5}$, and $\mathbf{1 6} .^{121,123,127-129,131}$ The aim of such studies was the development of tight-binding inhibitors, which, beyond establishing the canonical interactions of the benzensulfonamide moiety with the $\mathrm{Zn}^{2+}$ ion and its neighboring residues, would be able to interact with hydrophobic "patches" present at the border of the CA II active site cavity. ${ }^{121,123,127-129,131}$ Such hydrophobic patches were defined by the molecular surfaces of Phe131 on one hand and of the three adjacent residues, Leu198, Pro201, and Pro202 on the opposite face. Structural studies ${ }^{121,127}$ showed that, when these compounds interacted with the enzyme active site, the oligopeptide tails did not engage in any intermolecular hydrogen bond but established only van der Waals interactions with the aforementioned hydrophobic patches. At the same time kinetic studies ${ }^{121}$ suggested that such hydrophobic interactions contributed to improve the binding constants by a factor $10^{2}-10^{3}$ with respect to the simple 4-sulfamoylbenzamide, even though the strength of interaction was rather insensitive to the chemical structure of the tail. Finally, attempts to design molecules capable of binding simultaneously both hydrophobic patches did not lead to inhibitors with higher CA affinity than those able to bind these sites individually, 
highlighting once again the difficulties to predict effects of chemical structure on inhibitory properties.

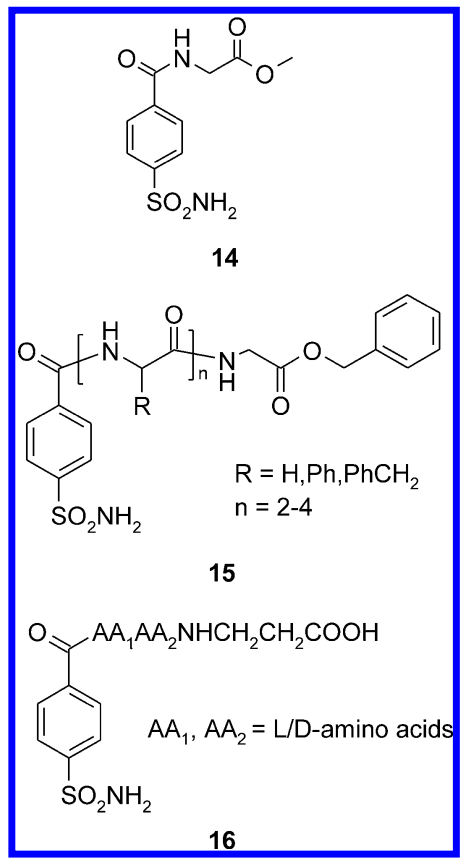

Subsequently, Supuran and co-workers reported another series of compounds where the derivatization of 12 and $\mathbf{1 3}$ was obtained by reaction with amino acid/dipeptide tails. ${ }^{120}$ These compounds showed nanomolar affinity for CA II and CA IV, the two $\mathrm{CA}$ isoforms involved in aqueous humor secretion within the eye. Since some of these compounds had a very good penetrability through the cornea, as a result of their good hydrophilicity/lipophilicity balance, they were easily formulated as eye drops either in solution or as suspensions, showing very good in vivo efficacy in animal models of glaucoma as intraocular pression lowering agents. ${ }^{120}$

Another kind of benzenecarboxamide derivatization was obtained with the attachment of oligoethylene glycol units to 12, generating CAIs of type $17 .{ }^{128}$ The effect of the length of repeating ethylene glycol (EG) units was kinetically investigated. These studies showed that dissociation constants increased only very slightly on increasing the number of the EG units, despite the fact that the inner $(\mathrm{EG})_{n}$ moieties $(n=$ $1-3$ ) interacted sufficiently with the protein to influence proton relaxation times, thus suggesting that eventual improvement in $\Delta H$ could be canceled by corresponding worsening in $\Delta S .^{128,129}$ Further derivatization of 17 with amino acid residues led to compounds of type $18 .^{129}$ Some of these last compounds were studied in their association with CA II by X-ray crystallographic methods showing that the observed differences in the values of the dissociation constants were not due to differences in metal ion coordination or to a diverse binding mode of the EG moieties to the enzyme, but instead arised from the capability of the pendant amino acid to interact with the protein surface. Overall these studies suggest that structural variations of inhibitors even at sites very distant from the sulfonamide moiety can significantly affect binding affinities. ${ }^{129}$

In this context it can be included also a recent study of Srivastava and co-workers, where the derivatization of $\mathbf{1 2}$ with a functional group supposed to interact with the surface exposed His residues of the CA enzymes, ${ }^{124,125,130}$ has allowed to obtain very strong CA inhibitors of type 19 and $20 .^{124,125}$ In these molecules the benzenesulfonamide is conjugated to the

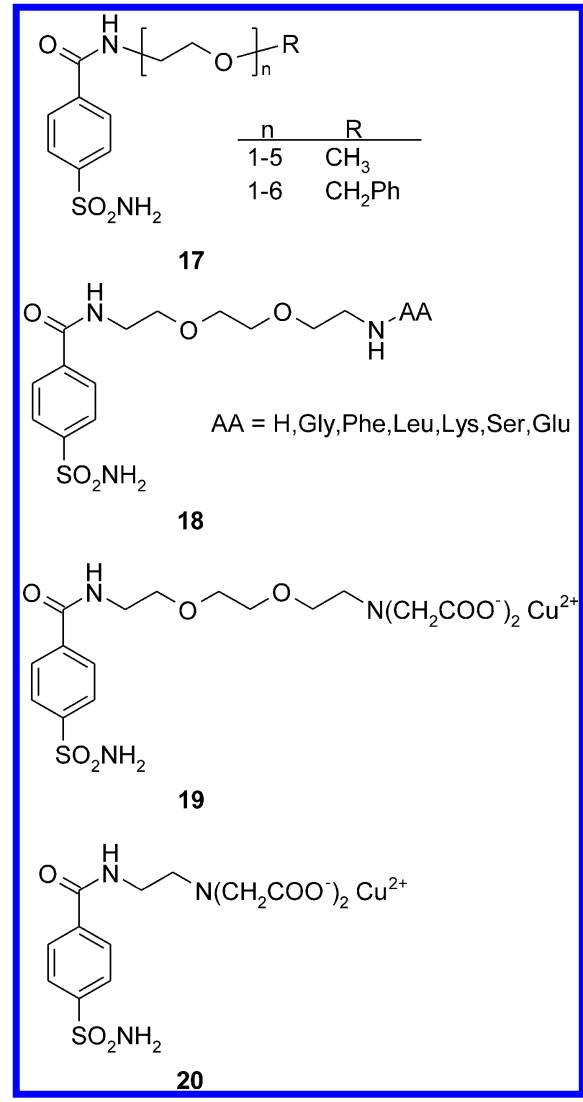

iminodiacetate- $\mathrm{Cu}^{2+}\left(\right.$ IDA-Cu$\left.{ }^{2+}\right)$ moiety via a spacer group, enhancing significantly the enzyme-inhibitor affinity. This "two-prong" approach was expected to provide a general strategy for converting weak inhibitors of enzymes into tightbinding ones. X-ray crystallographic studies of the hCA II/20 adduct (Figure 14) revealed that, as expected, the benzenesulfonamide moiety was coordinated to the $\mathrm{Zn}^{2+}$ similarly to other benzenesulfonamide inhibitors, while the IDA- $\mathrm{Cu}^{2+}$ prong was bound to His64, thus demonstrating that the high inhibition power of the inhibitor against CA II was related, in

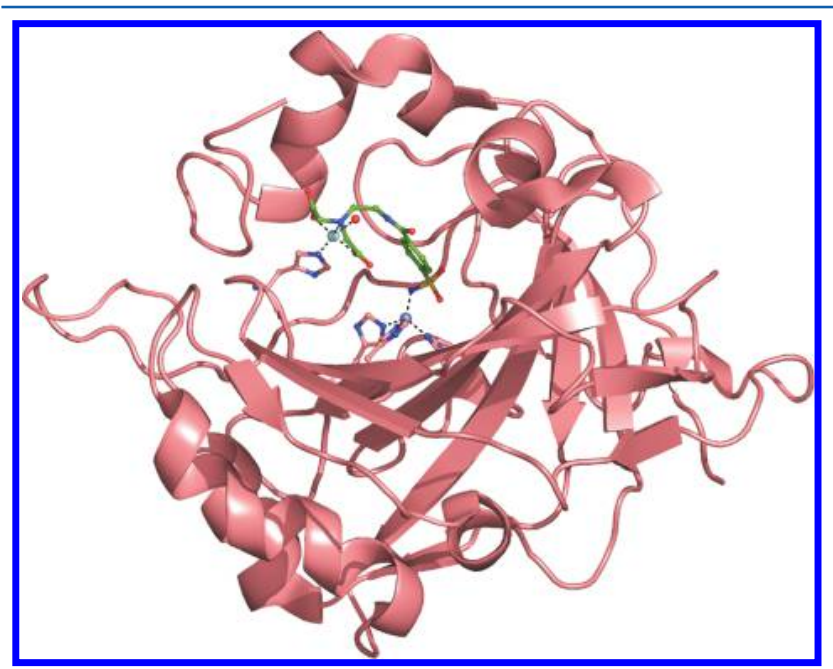

Figure 14. Ribbon diagram of the hCA II/20 complex (PDB code 2FOV). The inhibitor benzenesulfonamide moiety is coordinated to the catalytic zinc ion, while its IDA- $\mathrm{Cu}^{2+}$ prong is bound to His64 residue that adopts the "in" conformation. 


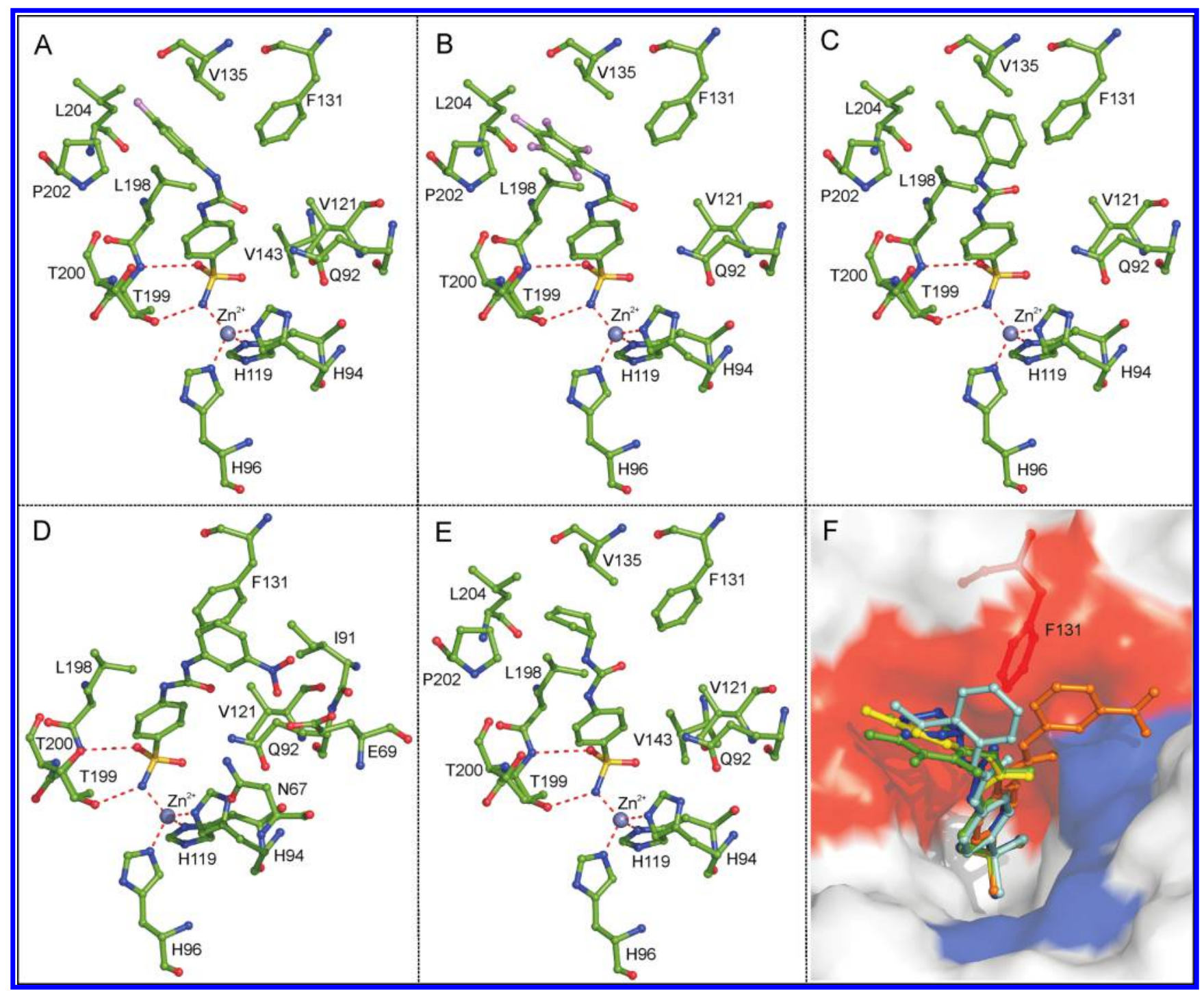

Figure 15. Active site representation of hCA II in complex with (A) 23 (PDB code 3N4B), (B) 24 (PDB code 3NON), (C) 25 (PDB code 3N3J), (D) 26 (PDB code 3N2P), and (E) 27 (PDB code 3MZC). (F) shows the superposition of the five ureido-derivatives (23 in yellow, 24 in green, 25 in cyan, 26 in orange, and $\mathbf{2 7}$ in blue). hCA II is represented as a surface-model (hydrophobic region, red; hydrophilic region, blue). Phe131 is also reported as ball-and-stick in red.

addition to the displacement of the catalytic nucleophile, to the inhibition of the catalytic proton shuttle His64. ${ }^{125}$

4.4.1.3. Benzenesulfonamides Containing Ureido and Thioureido Moieties. Benzenesulfonamides containing an ureido or thioureido moiety of type $\mathbf{2 1}$ and $\mathbf{2 2}$ represent an interesting class of recently reported CAIs, which possess peculiar CA inhibitory properties. ${ }^{133-137}$ Indeed, initial kinetic experiments showed that many of these molecules, differently from most of the previously reported sulfonamide CAIs, presented selective inhibition of hCA I over hCA II, thus representing good lead molecules for developing more selective CA I inhibitors. ${ }^{138}$ Nevertheless, up to now this class of molecules has been only poorly characterized from a structural point of view and a few structure-function relationship studies are available. In the ureido-derivative class, compounds 23-27, which have been obtained by reaction of 4-aminobenzenesulfonamide $\mathbf{5}$ with different aryl/alkyl isocyanates, ${ }^{137}$ are among the most thoroughly investigated examples. Inhibition assays against hCA II showed for these structurally related molecules a great variability in the $K_{\mathrm{I}}$ values, which spanned the range 3-226 nM. ${ }^{137}$ The structural reasons of such variability were investigated by solving the X-ray structures of all these derivatives in complex with hCA II. ${ }^{137}$ The comparative analysis of these structures showed that all the benzenesulfonamide groups of the five inhibitors established the canonical interactions with the enzyme active site and were rather well superimposable. On the contrary, the various tails presented different orientations, even if all pointed toward the exterior of the active site. In particular, in the molecules 23, 24, and 27 the 4-fluorophenyl, pentafluorophenyl and cyclopentyl groups were oriented toward the hydrophobic region delimited by residues Val135, Leu198, and Leu204 (Figure 15A,B,E). In molecule 26 the 3-nitrophenyl moiety was still located in a hydrophobic pocket, delimited by residues Ile91 and Val121, but which was separated from that occupied by the tails of 23,24 , and 27 by residue Phe131 (Figure 15D,F). Finally, the 2-isopropylphenyl tail of compound $\mathbf{2 5}$ was involved into an edge-to-face interaction with Phe131, assuming a position intermediate between those observed for 23,24 , and 27 on one side and 26 on the other (Figure 15C,F). ${ }^{137}$ The great variability observed 
in the orientations of all these tails within the hCA II active site could be ascribed to the high flexibility of the ureido fragment. Indeed, the torsion angles between the benzensulfonamide group and the second ring (see Figure 15A,B,C,D,E) were very different among all the five investigated compounds. Thus, the high flexibility of the ureido linker could allow the inhibitor to adopt different conformations, selecting the most energetically favored hydrophobic region to bind. ${ }^{137}$

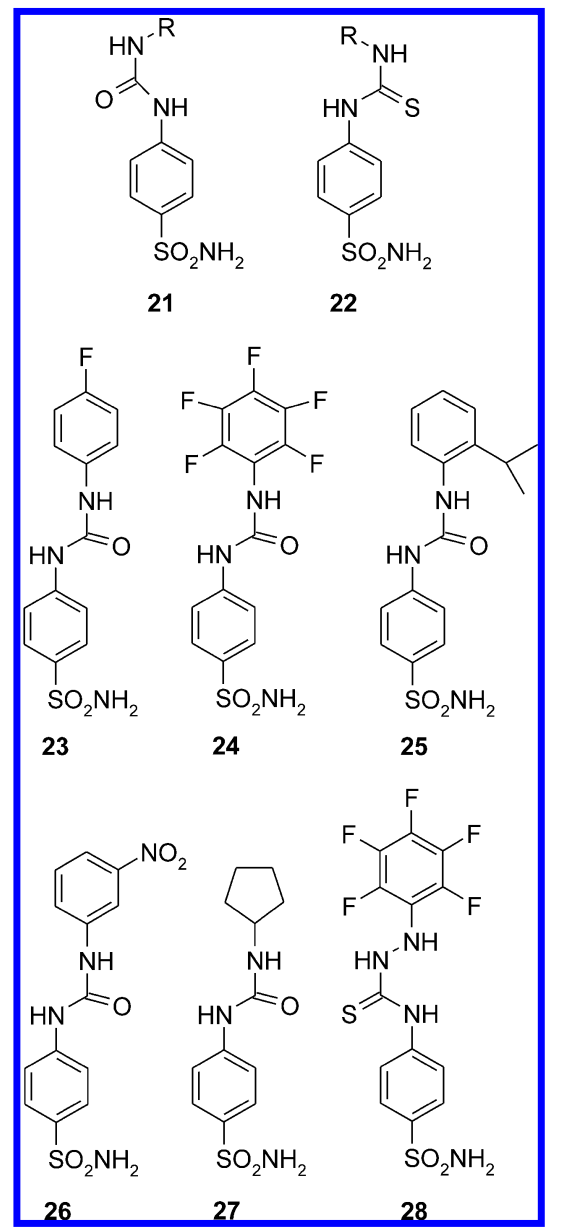

The structurally related thioureas of type $\mathbf{2 2}$ can be obtained from isothiocyanatosulfonamides and amines, hydrazines or amino acids. ${ }^{133-136}$ Some of these compounds have shown interesting features such as a good water solubility and a potent inhibitory activity against the ubiquitous isozyme hCA II and the transmembrane, tumor-associated hCA IX, representing thus interesting candidates for the design of antiglaucoma/ antitumor agents. ${ }^{133-136}$ However, as for the ureido-derivatives, only a few structural data on the binding mode of these molecules to the $\mathrm{CA}$ active site have been reported so far. ${ }^{139-141} \mathrm{~N}$-1-(4-Sulfamoylphenyl)- $N$-4-pentafluorophenylthiosemicarbazide $\mathbf{2 8}$ is an interesting member of this class, which has been demonstrated to act as an efficient inhibitor for hCA I ( $K_{\mathrm{I}}$ value of $\left.78 \mathrm{nM}\right)$, and very good for hCA II and hCA IX ( $K_{\mathrm{I}}$ values of 19 and $15 \mathrm{nM}$, respectively). ${ }^{139}$ The X-ray crystal structure of hCA II/ 28 complex showed the canonical binding mode of the benzenesulfonamide group to the active site with the thioureido moiety making strong van der Waals contacts with residues Phe131, Val135, Leu198, Pro202, and Leu204 (Figure 16A), and the pentafluorophenylamino tail, pointing toward the same hydrophobic pocket occupied by the tail of compounds 23, 24, and 27 (Figure 16B). ${ }^{139}$

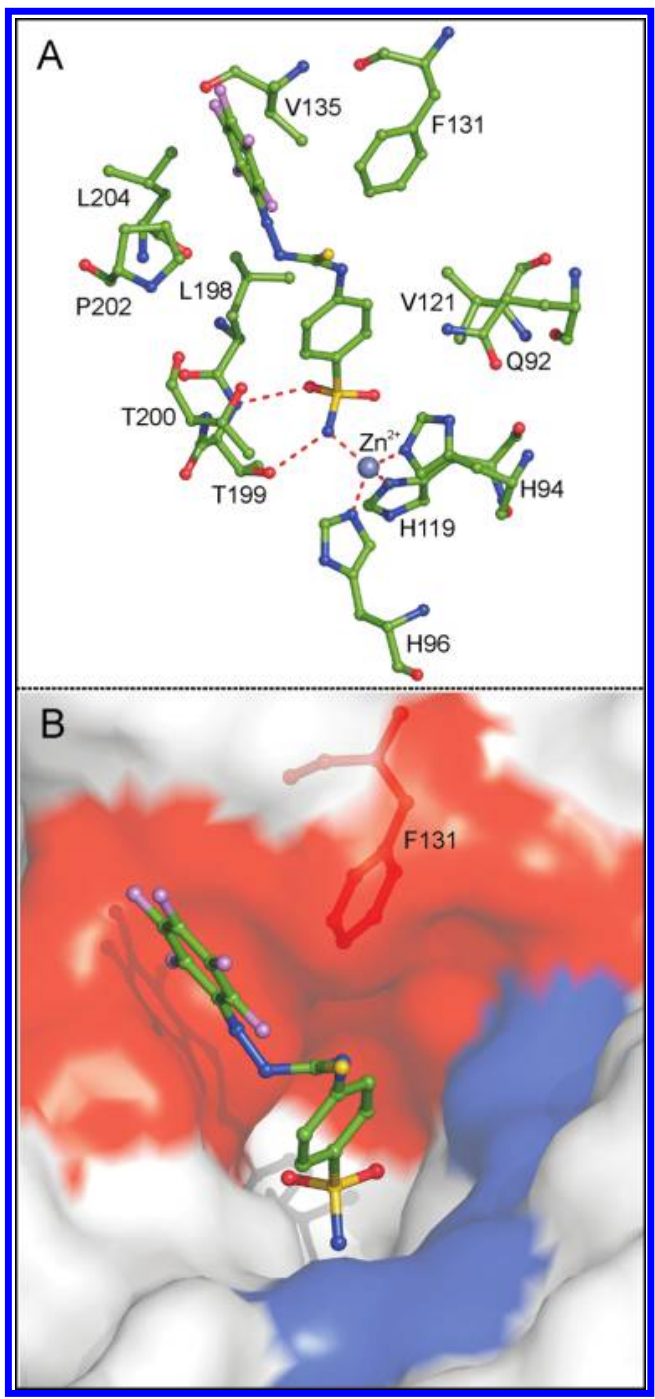

Figure 16. (A) Active site region in the hCA II/28 adduct $^{139}$ showing residues participating in recognition of the inhibitor molecule. Hydrogen bonds and the catalytic $\mathrm{Zn}^{2+}$ coordination are also shown. (B) View of the tail orientation of 28 within hCA II active site. The enzyme is represented as a surface-model (hydrophobic region, red; hydrophilic region, blue). Phe131 is also shown as ball-and-stick in red.

Unfortunately, the absence of structural studies on other thioureido compounds, possessing different tails and linkers, does not allow a detailed analysis of the potential binding sites of the tails as well as of the flexibility of the linker. Further studies are necessary to clarify this point.

4.4.1.4. Charged Benzenesulfonamides. As already mentioned in the Introduction, although many benzenesulfonamide CAIs possess high affinity for the different CA isoforms, the critical challenge for the design of new pharmacological agents is constituted by the lack of specificity of such compounds. An interesting approach to overcome at least in part this problem was proposed by Supuran and co-workers. ${ }^{142-148}$ Indeed, these authors suggested that sulfonamides with a permanent positive charge, due to their cationic nature, would be unable to penetrate through biological membranes and therefore would inhibit in vivo only CA enzymes located on the outer surface of the membranes, such as the extracellular isoforms CA IV, IX, XII, and XIV. Thus, a large series of such compounds was prepared by reaction of aromatic sulfonamides possessing a primary amino, imino, hydrazino, or hydroxyl group with 
pyrylium salts, affording compounds of type 29 and 30. ${ }^{145-147,149}$ These compounds were demonstrated to efficiently inhibit in vitro several CA isoforms, thus not showing any significant isozyme selectivity. ${ }^{142,143,146,148}$ However, in vitro and ex vivo experiments demonstrated that such derivatives were unable to cross the plasma membrane, confirming the design hypothesis. ${ }^{143,146,147}$ Such data are extremely important for specific in vivo inhibition of membrane-associated CA isozymes, especially the tumor associated CA IX and CA XII, and consequently for the eventual development of novel anticancer drugs.

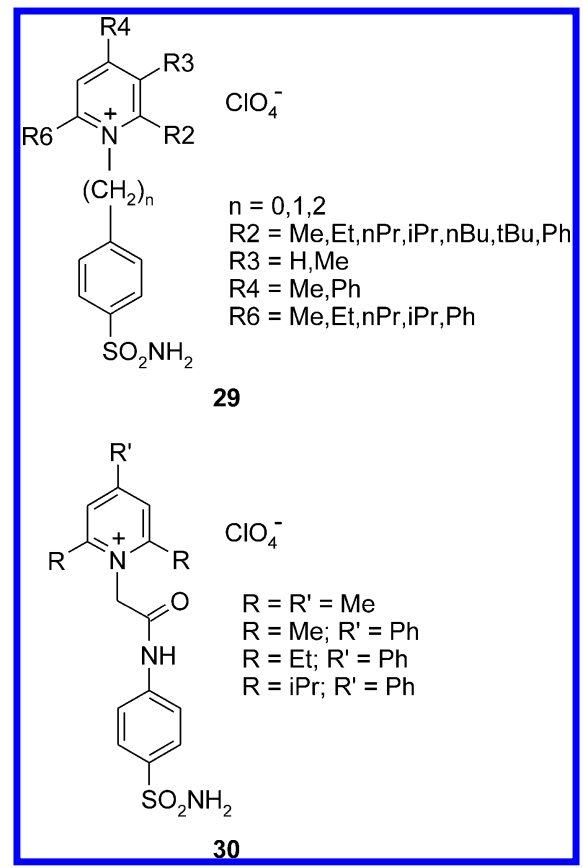

1-N-(4-Sulfamoylphenyl-ethyl)-2,4,6-trimethylpyridinium perchlorate $31^{142,146,150}$ is one of the most interesting derivatives of this series. Indeed, although kinetic studies demonstrated that this molecule is able to inhibit several CA isoforms in the nanomolar range $\left(K_{\mathrm{I}}\right.$ values of 4,21 , and $14 \mathrm{nM}$ against CA I, II, and IX, respectively), ${ }^{142}$ because of its membrane impermeability, in vivo it could interact only with membrane-associated isozymes without appreciable inhibition of the cytosolic isoforms. ${ }^{150}$ More interestingly, 31 was also shown to diminish in vitro the acidification of hypoxic tumors overexpressing CA IX, thus constituting an interesting lead compound for the design of antitumor sulfonamides. ${ }^{151}$

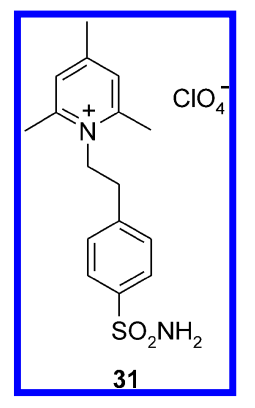

The X-ray crystal structure of the hCA II/31 complex was solved, providing interesting insights into the molecular basis responsible for its CA affinity. ${ }^{150}$ In particular, the analysis of this structure showed that while the benzenesulfonamide moiety was involved in the canonical interactions within the active site cavity, the 2,4,6-trimethylpyridinium moiety established several other important interactions. Indeed, this group was located in the same hydrophobic pocket occupied by the tail of ureido-derivative 26, making van der Waals interactions with Ile91 and establishing a strong face-to-face stacking interaction with Phe131 side chain (Figures 15D and 17). These findings, together with those reported in the

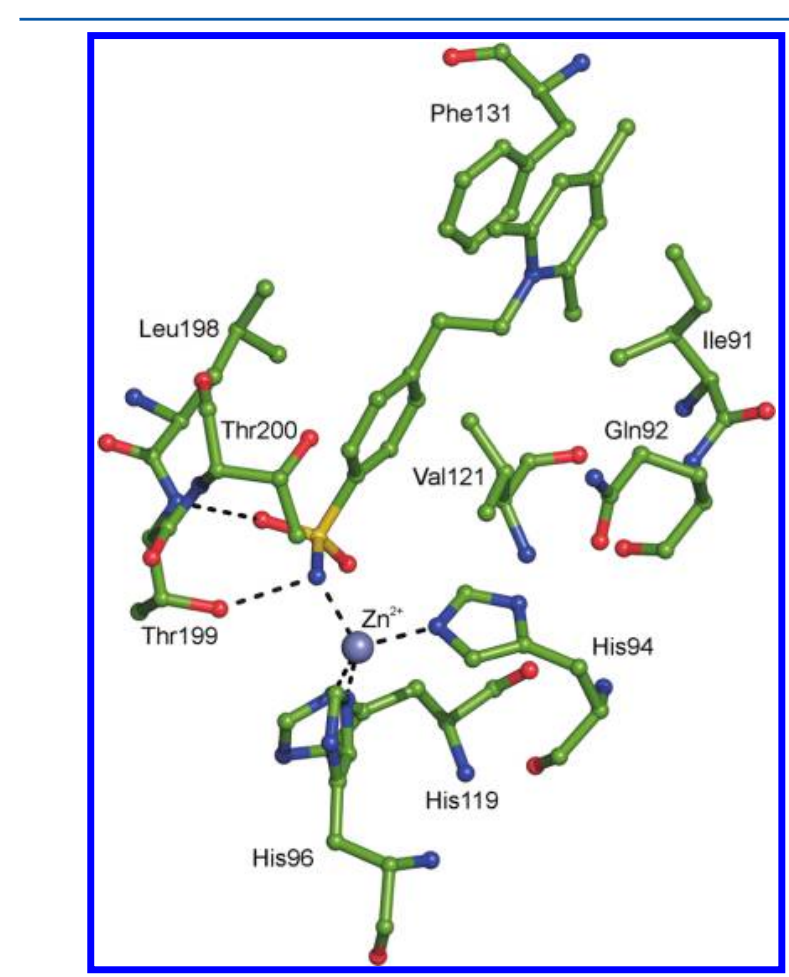

Figure 17. Representation of active site region in the hCA II/31 complex (PDB code 1ZE8) showing hydrophobic residues involved in the recognition of the inhibitor molecule (distance $<4.5 \AA$ ). Hydrogen bonds and the active site $\mathrm{Zn}^{2+}$ coordination are also shown.

previous paragraph, evidenced a key role for residue Phe131 in the orientation of the inhibitor molecule in the CA II active site. Since the different CA isozymes present diverse amino acids in position 131, this residue could represent a target to achieve inhibitors with higher isozyme selectivity.

The effect of the presence of a positive, negative or neutral substituent on benzenesulfonamide CAIs has been investigated in detail also by Srivastava's group. In this case, the aim was to understand the effect of different charges in the CA I and CA II active sites, to discriminate the binding to these two isoforms. ${ }^{152}$ In particular, four simple benzenesulfonamide inhibitors, specifically compounds $32-35$, substituted at the para position with differently charged functional groups, were synthesized. The substituents were chemically different and were designed to bind in the midsection of the active site, in order to explore their features also as possible molecular linkers connecting the benzenesulfonamide moiety and a second functional group in a "two prong" approach (see section 4.4.1.2). The affinities and X-ray crystal structures of the complexes that these compounds formed with hCA I and hCA II were determined, revealing that a positively charged amino group was more poorly tolerated in the active site of CA I compared with CA II, while a negatively charged carboxylate substituent was tolerated equally well in the two active sites. However, in both cases enzyme-inhibitor affinity improved 
upon neutralization of inhibitor charged groups by acylation or esterification. ${ }^{152}$ These studies provided important indications into the choice of molecular linkers that could potentially be used to alter the binding properties of bifunctional inhibitors. ${ }^{152}$

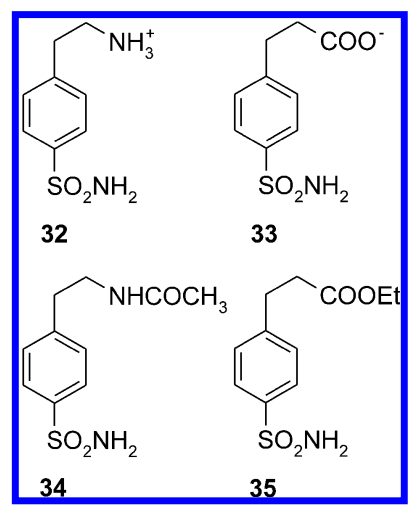

4.4.1.5. Benzenedisulfonamides. The presence of a second sulfonamide group in benzenesulfonamide derivatives has been largely investigated both in the search of more active CAIs and to develop compounds with a different biological activity (the CA inhibitory action being less important in the second case)..$^{53}$ Two different types of such derivatives have been so far characterized: those where the two sulfonamide moieties are present on the same phenyl ring (see compounds 36-38) $)^{153}$ and those where the two sulfonamide moieties are present on two different phenyl rings, separated by urea, guanidine, carboxyamido moieties etc., as spacers (see compounds 39). ${ }^{53,154,155}$

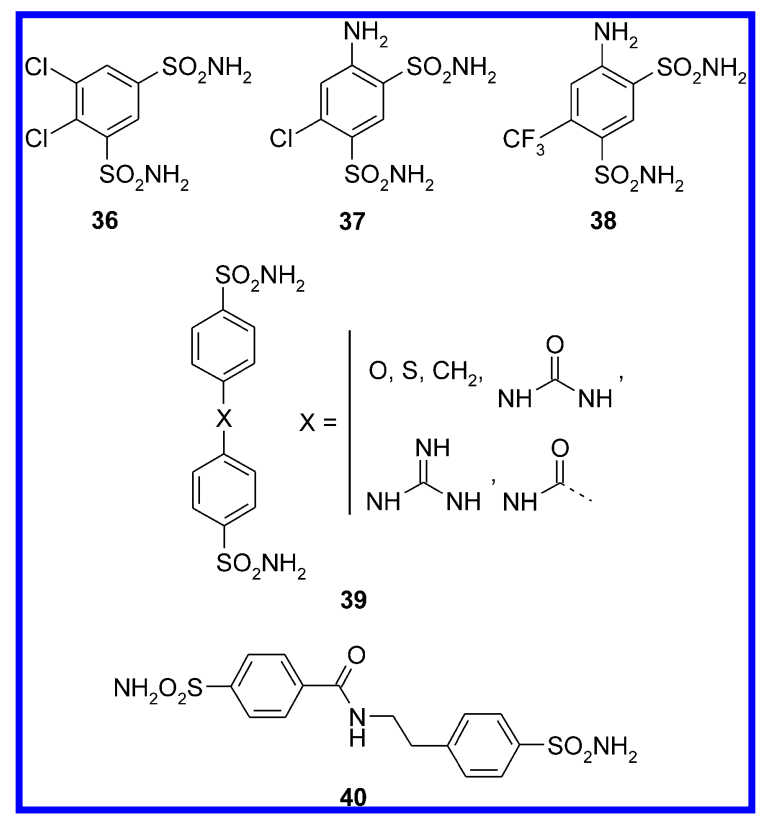

Dichlorophenamide 36, which has been largely clinically used for the treatment of glaucoma ${ }^{15,114,156}$ and several neurological disorders, ${ }^{114,157}$ and its closely related analogues 6-chloro-4amino-benzene-1,3-disulfonamide 37 and 6-trifluoromethyl-4amino-benzene-1,3-disulfonamide $\mathbf{3 8}$ are examples of the first type of such derivatives. These compounds, that were all demonstrated to act as nanomolar inhibitors of hCA II, were extensively characterized from a structural point of view. ${ }^{153}$ In particular, the high resolution structures of these three compounds in complex with hCA $\mathrm{II}^{153}$ revealed for all of them a particular binding mode within the enzyme active site.
Figure 18 shows the key interactions which stabilize the binding of these compounds to the enzyme. In particular, beyond the canonical coordination of the $\mathrm{Zn}^{2+}$ ion by means of the ionized sulfonamide $\mathrm{NH}^{-}$group, the substituted phenyl moiety of each inhibitor makes various van der Waals and hydrogen bond interactions with residues delimiting the cavity. In all three complexes the meta-sulfonamide group is oriented toward the hydrophilic side of the active site cleft and establishes several hydrogen bond interactions. Such interactions force the plane of the phenyl moiety to assume an orientation rotated by $\sim 45^{\circ}$ and tilted by $\sim 10^{\circ}$ with respect to that generally observed in other CA II/benzenemonosulfonamide complexes ${ }^{53}$ and described in section 4.4.1 (Figure 19). These findings suggest that the presence of an additional sulfonamide group in metaposition of benzenesulfonamide CAIs can be utilized to differently position the phenyl moiety within the active site and to opportunely orient a putative tail of the phenyl ring, allowing the exploration of active site regions different from those usually occupied by tails of the classical benezenemonosulfonamide inhibitors. ${ }^{153}$

4-(4-Sulfamoylphenylcarboxamidoethyl)benzenesulfonamide 40 is a representative compound of the class of bissulfonamides of type 39. Such a molecule has been demonstrated to act as a topically acting antiglaucoma drug, with strong inhibition properties toward hCA II $\left(K_{\mathrm{I}}\right.$ of 5 $\mathrm{nM}){ }^{120}$ The X-ray crystal structure of the hCA II/40 adduct has been solved, providing insights into the interaction mode of this type of inhibitors with the enzyme. ${ }^{155}$ Indeed, the structural analysis highlighted that the two sulfamoyl moieties of the inhibitor allow for its optimal orientation within the active site, with the first one which binds in ionized form to the zinc ion, the organic part lying in the hydrophobic region of the active site and with the terminal, carboxyamido containing phenylsulfamoyl head participating in strong hydrogen bonds with residues located at the entrance of the active site cavity (Figure 20). ${ }^{155}$ On the basis of these findings it is conceivable that modifications of organic scaffold of $\mathbf{4 0}$ (such as the substitution pattern of the two phenyl rings, the nature and the length of the linker between them etc.), designed to optimize the interaction with the different residues present in the active site of the diverse CA isoforms, could in principle lead to more selective CAIs with different type of applications. ${ }^{155}$

4.4.1.6. Benzenesulfonamides Derivatized with Fluorescent Probes. In the past a lot of research on CAs was concentrated on the study of the involvement of these enzymes in different type of tumors. Such studies identified first hCA $\mathrm{IX}^{158}$ and later hCA XII ${ }^{159,160}$ as the CAs associated to tumors. Among these two isoforms, CA IX was considered of particular interest for its peculiar features; indeed this enzyme is present only in a limited number of normal tissues (mainly in the gastrointestinal tract) (see Table 1), whereas it is overexpressed in hypoxia and during development of various human carcinomas, ${ }^{161}$ thus representing a valuable target for cancer diagnosis and treatment. In this context, the ability to detect the overexpression of this protein, that identifies a population of abnormal cells, has the potential to improve the accuracy and efficacy of early cancer detection. Toward this goal, the selective recognition of this protein by different kinds of molecular probes, such as fluorescent, spin-labeled and ${ }^{129} \mathrm{Xe}-$ based probes, has been largely exploited in the last period. ${ }^{140,162,163}$ However, since the crystal structure resolution of CA IX has only recently been achieved, ${ }^{63}$ the rational design of such probes has been mainly performed by using the structural 


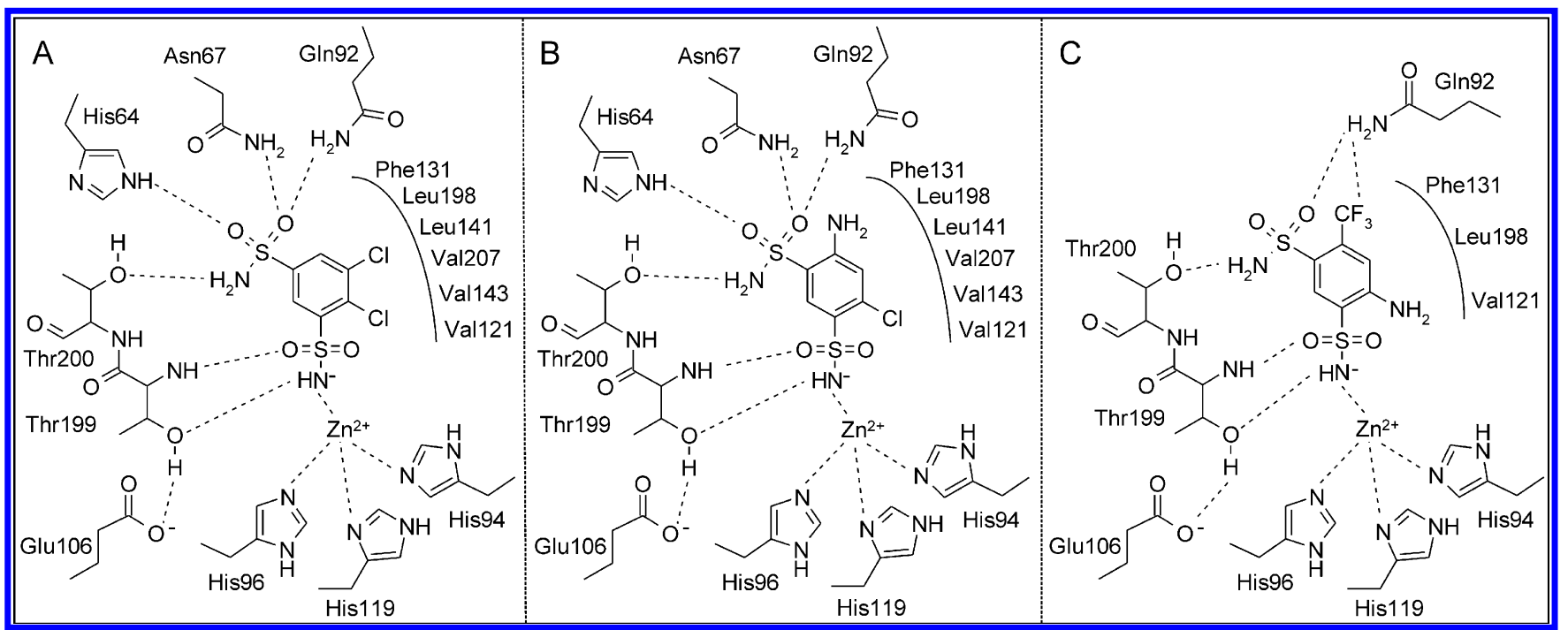

Figure 18. Schematic representation of the binding mode of sulfonamides 36 (A), 37 (B), and 38 (C) within the hCA II active site (PDB codes $2 \mathrm{POU}, 2 \mathrm{POV}$, and $2 \mathrm{POW}$ for 36,37 , and 38 , respectively).

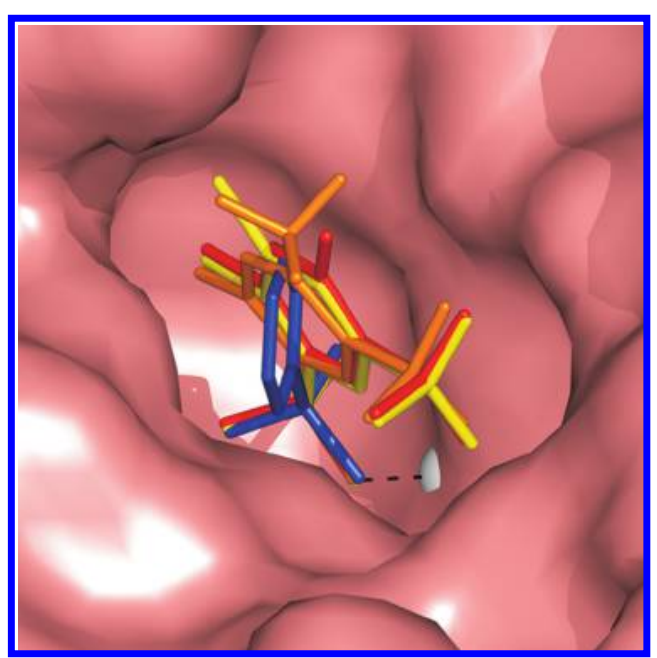

Figure 19. Superposition of benzenedisulfonamides 36-38 (36 yellow, 37 red, and 38 orange) with the benzenesulfonamide 6 (blue; PDB 2WEJ). hCA II is represented as a surface-model and the catalytic zinc ion as a gray sphere.

information derived from the most common and easily crystallizable isoform, that is, CA II. The rationale for developing such molecular probes was to attach moieties which confer specific physicochemical properties (e.g., fluorescence, free radical character, etc.) to sulfonamide CA inhibitors, which should preferentially bind to CA IX and XII. $^{151,163}$

The development of several fluorescent CAIs has been reported. Fluorescein is an optimal moiety for designing such compounds, since it presents an appropriate excitation and emission wavelength, when present in various biologically active derivatives (i.e., 495 and $519 \mathrm{~nm}$, respectively) and the synthesis of such compounds is rather straightforward. Preliminary studies on this topic were reported in 1996 by Christianson's group. ${ }^{164}$ In particular, starting from the crystal structure of the CA II/arylsulfonamide 41 complex, ${ }^{164}$ these authors adopted an iterative structure-based procedure for the rational design of the fluorescein-derivatized arylsulfonamide probe 42. However, in the three-dimensional structure of the

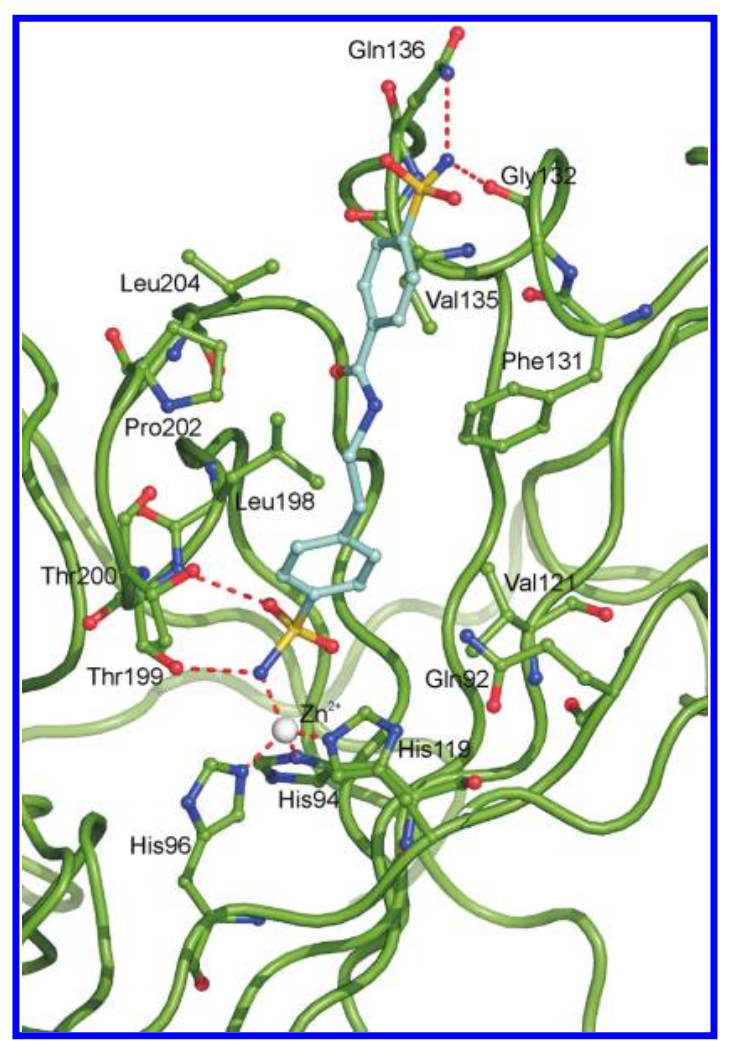

Figure 20. View of the active site region in the hCA II/40 complex showing residues participating in recognition of the inhibitor molecule. Hydrogen bonds and the active site $\mathrm{Zn}^{2+}$ coordination are also shown. The PDB coordinate file has been kindly provided by Claudiu $T$. Supuran.

CA II/42 complex, although $\mathbf{4 2}$ was able to tightly bind to the enzyme $\left(K_{\mathrm{d}}=2.3 \mathrm{nM}\right)$, the fluorescein moiety was not able to establish direct interactions with it. The strength of binding was therefore attributable only to the canonical interactions of the benezenesulfonamide moiety and to some hydrophobic interactions of the linker, connecting the fluorescein and the benzenesulfonamide moieties, with residues Leu198, Pro202, and Phe $131 .{ }^{164}$ The analysis of the structure suggests that the 
absence of a direct interaction of the fluorescein moiety with the active site could be related to the excessive length of the linker which brings the fluorescein moiety out of the active site cavity.

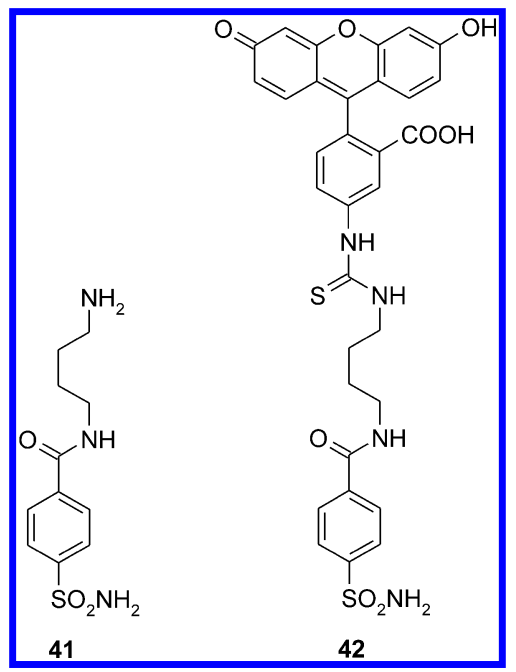

More recently Supuran's group reported the development of a new series of fluorescent sulfonamides of type $43 \mathbf{a}-43 \mathbf{j},{ }^{163}$ characterized by the presence of a fluorescein moiety, a benzenesulfonamide variously substituted, and a thioureido moiety as linker. One of the most interesting compounds in this series was the derivative (4-sulfamoylphenylethylthioureido)fluorescein (43h). ${ }^{140}$ Indeed, this derivative, besides showing strong capability to bind the cytosolic hCA II isozyme $\left(K_{\mathrm{d}}=\right.$ $0.64 \mathrm{nM}),{ }^{140}$ presented even higher affinity for the tumorassociated CA IX $\left(K_{\mathrm{d}}=0.30 \mathrm{nM}\right) .{ }^{140}$ For this reason, it is actually in clinical studies as an imaging tool for acute hypoxic tumors. ${ }^{151}$ The X-ray crystal structure of $43 \mathrm{~h}$ in complex with the cytosolic isoform hCA II has been reported together with a modeling study of the adduct which this inhibitor forms with the isoform hCA IX. ${ }^{140}$ The crystallographic analysis showed that, beyond the canonical interactions of the benzenesulfonamide group within the hCA II active site, the thioureido moiety established strong van der Waals interactions with residues Gln92, Val121, Phe131, Val135, Leu198, Thr199, Thr200, and Pro202 (Figure 21A). Moreover, differently from what observed for compound 42, in this case the bulky fluorescein moiety was well-defined in the electron density maps, being located at the rim of the active site, on the protein surface, strongly interacting with the $\alpha$-helix formed by residues Asp130-Val135 (Figure 21B). Molecular modeling studies of the corresponding adduct with hCA IX revealed that all these interactions were preserved in the binding to hCA IX, even if an additional polar interaction was observed. In particular, in the latter case the carbonyl moiety of the fluorescein tail participated in a strong hydrogen bond with the guanidine moiety of Arg130, an amino acid characteristic of the hCA IX active site (Figure 22). This interaction is probably responsible for the roughly twice higher affinity of $43 \mathrm{~h}$ for hCA IX over hCA II and could explain why in vivo the compound specifically accumulated only in hypoxic tumors overexpressing CA IX and not in the normal tissues. ${ }^{140}$

4.4.1.7. Benzenesulfonamides Derivatized with SpinLabeled Probes. Electron paramagnetic resonance (EPR) techniques have been largely used for the investigation of protein conformations and their changes in different milieus. ${ }^{165,166}$ Moreover, such techniques can be also utilized to study inhibitor binding to an enzyme active site, since such molecules have different mobility depending on whether they are free in solution or they are bound to the target enzyme, and can therefore show major changes in their EPR spectrum when complexed to the enzyme. For these reasons, in recent years a great attention has been dedicated to the development of CAIs containing a free-radical moiety active in EPR measurements. The development of such inhibitors, if associated to a certain degree of selectivity for isoform CA IX, can represent an

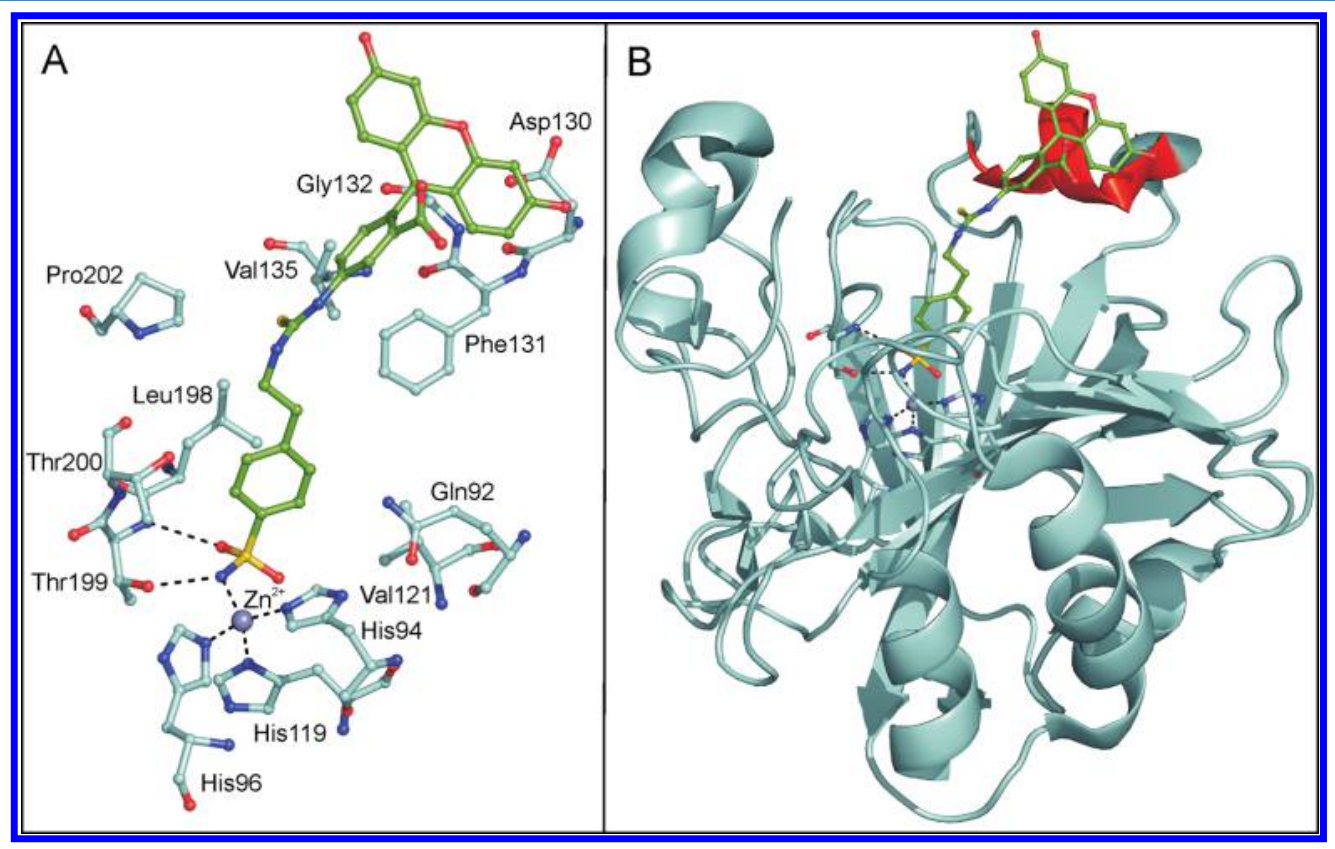

Figure 21. (A) Representation of $\mathbf{4 3 h}$ binding within the hCA II active site (PDB code $2 \mathrm{~F} 14$ ). (B) Ribbon diagram of the hCA II/43h adduct showing as the fluorescein moiety, located at the rim of the active site, strongly interacts with the $\alpha$-helix formed by residues Asp130-Val135 ( $\alpha$-helix is colored in red). 


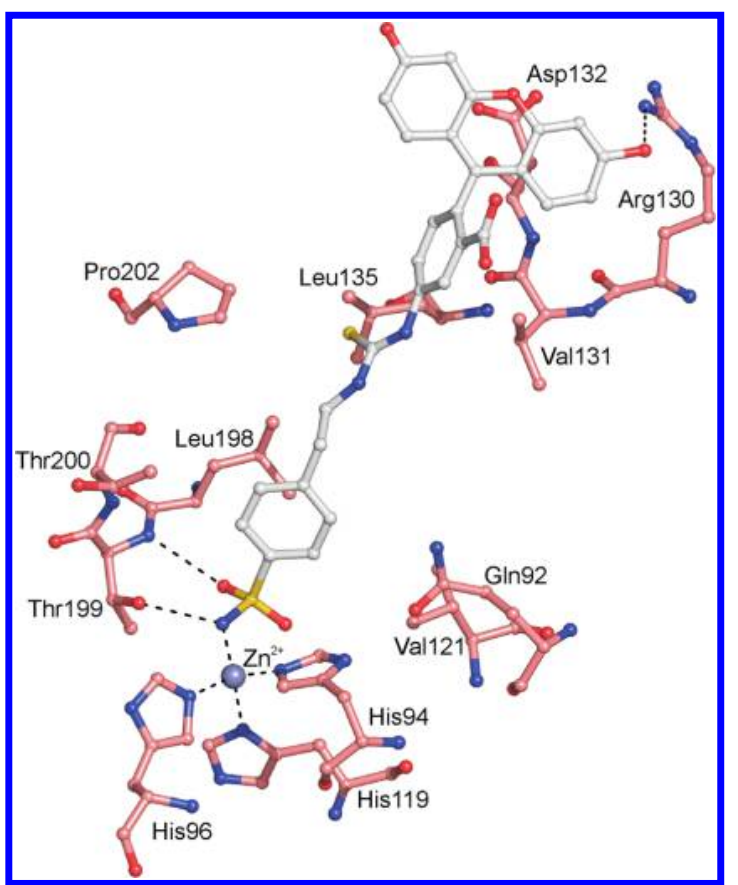

Figure 22. View of the active site of the hCA IX/43h model showing residues participating in the recognition of the inhibitor molecule. $\mathrm{Zn}^{2+}$ coordination and hydrogen bonds are also shown.

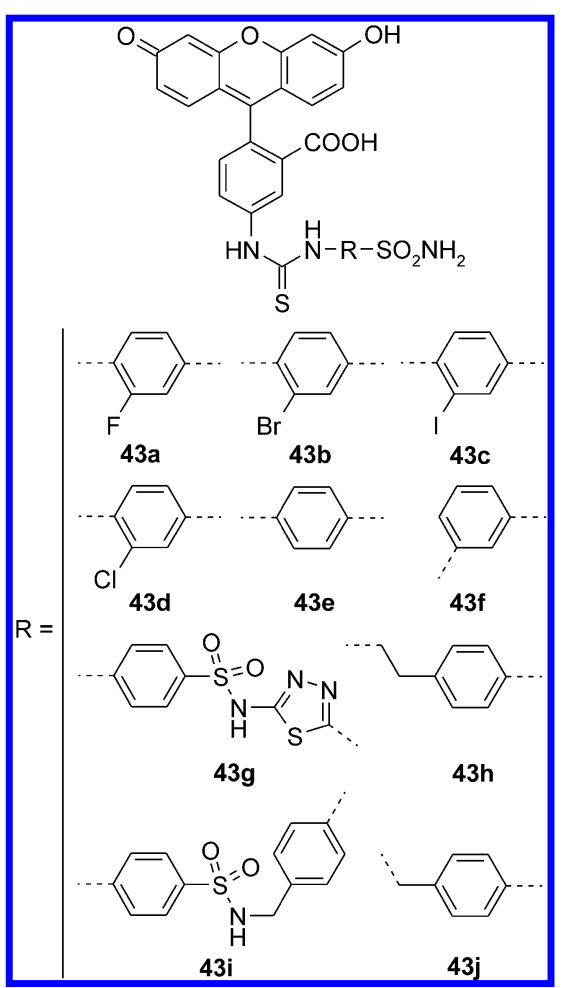

interesting tool for the investigation of hypoxic tumors by EPR techniques.

Preliminary studies on spin-labeled CAIs were reported in 70 s with the aim to derive structural information on the CA active site, ${ }^{167-171}$ since in those years no structural information was available on any CA isoform alone or in complex with inhibitors. On the contrary, more recently, with the aim to investigate new strategies for the labeling of tumor associated CAs, a new series of derivatives $(\mathbf{4 4 a - 4 4 i )}$ has been generated by using the classical tail strategy. In such derivatives the spin- labeled 2,2,6,6-tetramethylpiperidine-1-oxyl (TEMPO) moiety is inserted as tail in a classical benezenesulfonamide scaffold, by using thiourea as linker. ${ }^{172}$

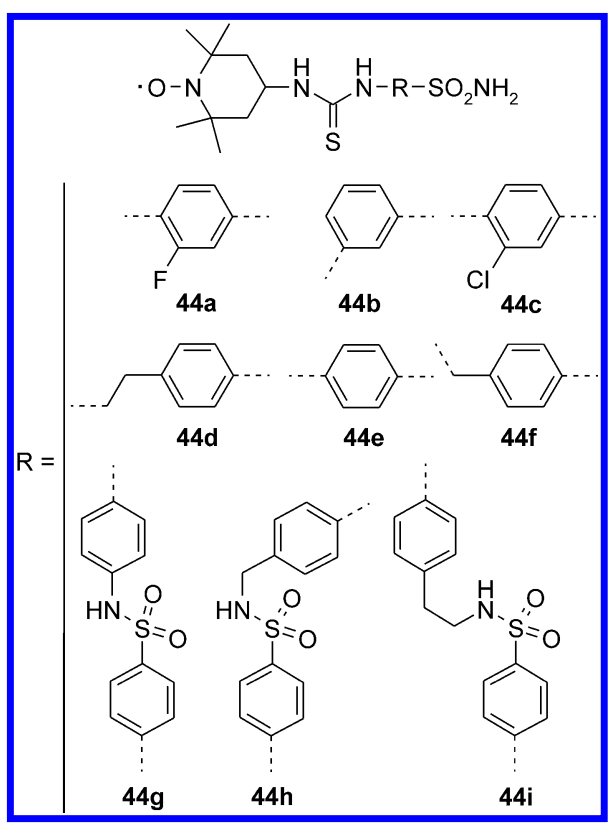

The TEMPO group was chosen as free radical moiety in these molecules for its advantageous features in EPR experiments. Indeed it possesses a simple EPR spectrum, ${ }^{173}$ shows a high stability, and induces a relatively good water solubility to the compounds incorporating it. Moreover, its spectral properties (such as line width and intensity) can be modulated in in vivo experiments by tissue oxygen or redox status. ${ }^{173,174}$

Compounds $44 a-44 i$ were tested for their inhibitory properties against hCA I, hCA II and hCA IX, showing generally an efficient inhibition of hCA II and hCA IX $\left(\mathrm{K}_{\mathrm{I}}\right.$ in the range of $12-76 \mathrm{nM}$ ) and only a moderate inhibition of hCA I ( $K_{\mathrm{I}}$ in the range of $\left.132-2070 \mathrm{nM}\right) .{ }^{175}$ The crystal structure analysis of $44 \mathrm{c}$, one of the best CA II inhibitors of the series, in complex with hCA II was also solved providing detailed information on the inhibition mechanism of this class of molecules. ${ }^{141}$ In particular, the structural analysis revealed that beyond the classical interactions of the benzenesulfonamide moiety, only a few additional hydrophobic interactions between the thioureido linker and the hydrophobic region of the active site were present (Figure 23). Indeed, the TEMPO moiety showed a certain degree of flexibility and thus was not entirely visible in the electron density maps. ${ }^{141}$

EPR spectra of three representative compounds of this series, namely 44b, 44c, and 44e, were recorded in the presence and in the absence of CA II. ${ }^{141}$ In absence of the enzyme the three spectra were all superimposable, in agreement with the fact that their structural differences were located at the benzenesulfonamide moiety, far from the free radical piperidin- $N$-oxide moiety. On the contrary, in the presence of hCA II, the variation of the EPR line shape indicates that the piperidine ring of the inhibitor molecule, due to the binding to the enzyme, loses its conformational freedom. Moreover, in agreement with the corresponding CA II inhibition constants, the amount of enzyme requested to reach the limit of the line shape was markedly different for the three sulfonamides: the hCA II/ inhibitor ratio was highest for compound 44b (hCA II/ inhibitor $>10$ and $K_{\mathrm{I}}$ of $\left.165 \mathrm{nM}\right),{ }^{175}$ and it did not change much for the other two inhibitors (hCA II/inhibitor $>0.7$ and 


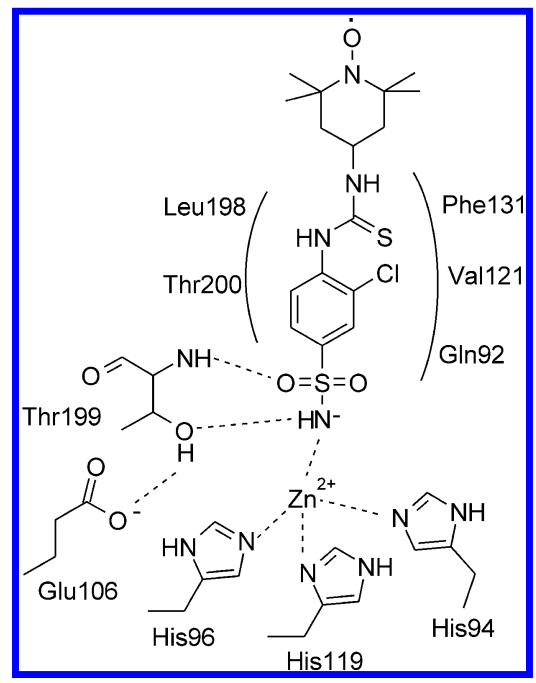

Figure 23. Schematic representation of the binding mode of $44 \mathrm{c}$ within hCA II active site (PDB code 3EFT). Residues participating in the recognition of the inhibitor molecule are also reported.

2; $K_{\mathrm{I}}$ of 12 and $41 \mathrm{nM}$ for $44 \mathrm{c}$ and $44 \mathrm{e}$, respectively). ${ }^{175} \mathrm{EPR}$ experiments were carried out also in presence of hCA IX, using two enzyme recombinant forms: the first consisted of the sole catalytic domain and the second of the catalytic domain and the proteoglycan (PG) fragment. This latter construct, representing the in vivo extracellular part of the protein, was useful to detect if the PG segment can modulate the interaction between the inhibitor and the enzyme active site in cells. In both cases, it was evident from the line-shape appearance, that the dynamics of the $\mathrm{N}-\mathrm{O}$ portion of the inhibitors were more hindered in complexes with hCA IX than with hCA II, thus suggesting that the hCA IX cavity was slightly smaller. The only difference observed between the EPR spectra measured with hCA IX catalytic domain alone and the longer recombinant form was that a higher hCA IX/inhibitor ratio was necessary in the latter case to reach the same EPR line shape. These results demonstrated that the PG domain does not hinder the binding of these molecules to the active site, although it can partially limit the access. ${ }^{141}$ These data were successively confirmed by the crystal structure resolution of the CA IX catalytic domain, ${ }^{63}$ which evidenced the location of the PG domain at the border of the active site, thus justifying a partial steric hindrance in the binding of bulky inhibitors. Altogether these data opened the way to the development of new probes to be used for imaging purposes by EPR techniques.

4.4.1.8. Benzenesulfonamides Derivatized with ${ }^{129} \mathrm{Xe}-$ Cryptophane Probes. Several investigations are currently underway on ${ }^{129} \mathrm{Xe}$ biosensors for their potential use as a magnetic resonance imaging (MRI) agents. The monatomic ${ }^{129} \mathrm{Xe}$ isotope has a spin $-1 / 2$ nucleus, exhibits a large chemical shift window, ${ }^{176}$ and can be laser polarized to enhance MRI signals $\sim 10000$-fold, ${ }^{177}$ making it an appealing biomolecular probe for MRI. Its current in vivo MRI applications are restricted to functional lung imaging through the diffusion of Xe gas; ${ }^{178}$ however, recent studies highlighted that encapsulation of ${ }^{129} \mathrm{Xe}$ within a cryptophane cage ${ }^{179}$ allows for its use as biosensor that can be targeted to specific proteins using an appropriate affinity tag. ${ }^{180,181}$

Chambers and co-workers applied these ideas to the design of ${ }^{129} \mathrm{Xe}$-cryptophane biosensors able to bind to CA isozymes. ${ }^{182}$ In these compounds a ${ }^{129} \mathrm{Xe}$ binding cryptophane was associated with linkers of different lengths to $p$ benzenesulfonamide, yielding non diastereomeric biosensors of type $45 a-45 c$ with dissociation constants for isoforms I and II in the nanomolar range. ${ }^{182}$ The most interesting feature of such compounds was that, when bound to the target enzymes (in the reported studies, CA II), they showed reproducible changes in ${ }^{129} \mathrm{Xe}$ chemical shifts of $5.5,7.5$, and $5.0 \mathrm{ppm}$, for $45 \mathrm{a}-45 \mathrm{c}$, respectively. ${ }^{182}$ It is worth noting that $7.5 \mathrm{ppm}$ is the largest chemical shift ever reported for a Xe biosensor. Unfortunately, such compounds have not been evaluated for their interaction with the tumor-associated isoforms, CA IX and XII.

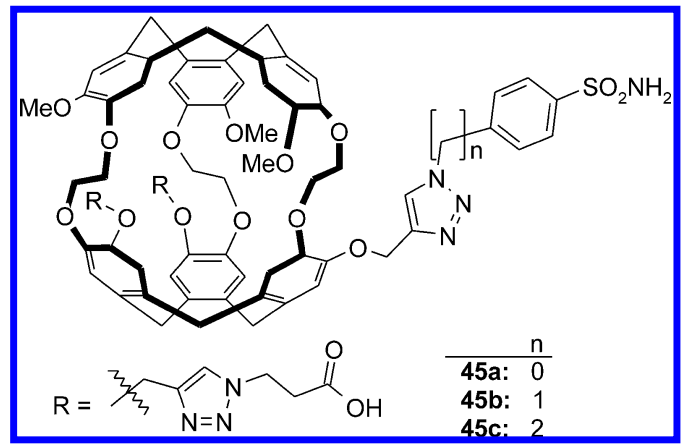

The structural details of the interactions with hCA II were derived by reporting the crystal structure resolution of the CA II/45c complex. ${ }^{162}$ This study showed the binding of the benzenesulfonamide moiety to the $\mathrm{Zn}^{2+}$ ion of the active site, and the bulky cryptophane moiety at the upper edge of the cavity (Figure 24). Altogether these biochemical and structural

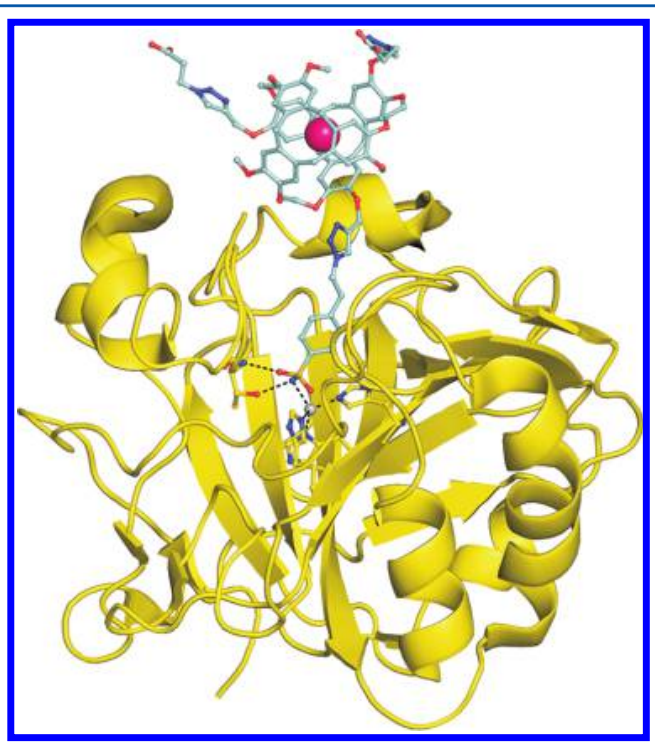

Figure 24. Ribbon diagram of the hCA II/45c complex (PDB code $3 \mathrm{CYU})$. The cryptophane-derived CA biosensor, the ${ }^{129} \mathrm{Xe}$ atom, the catalytic triad, the zinc ion and Thr199 residue are represented in balland-stick.

studies have provided the first experimental evidence of how an encapsulated ${ }^{129} \mathrm{Xe}$ atom can be targeted to a biomedically relevant protein, offering very important perspectives in the field of cancer diagnosis, although, as we have stressed above, no data for the interaction of these compounds with CA IX and XII have been provided so far. 
4.4.1.9. Hypoxia-Activatable Benzenesulfonamides. A prodrug is a pharmacological substance, which is administered in an inactive form, but can be metabolized in vivo into an active metabolite, through a process termed bioactivation. ${ }^{183}$ In the last years the employ of hypoxia-activatable prodrugs has been largely exploited for improving the selectivity of tumor cell killing. ${ }^{184}$ In particular, since, as reported above, hypoxic tumors overexpress massive amounts of CA IX, this enzyme has become a very interesting target for the design of hypoxiaactivatable CAIs. ${ }^{185-187}$ For this aim, different classes of compounds have been developed, ${ }^{185-187}$ among which disulfide derivatives of aromatic sulfonamides have shown the most promising results (compounds of type $46 a-46 d$ and $47 a-$ 47b). ${ }^{185}$, ${ }^{87}$ Such disulfide-containing sulfonamides are generally very bulky compounds and therefore unable to bind into the CA active site cavity. However, they can be reduced under the reducing conditions present in hypoxic tumors (see scheme 3 ), generating the corresponding thiol derivatives, which

Scheme 3. Reduction of Disulfide-Containing Sulfonamides to the Corresponding Thiol Derivatives

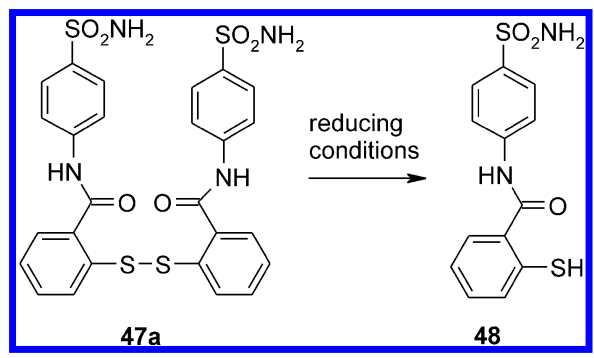

present the classical features of the most potent CAIs. The advantage in the use of this kind of molecules resides in the fact that, even if the reduced thiol form of the inhibitor does not present a high selectivity for isoform IX, since it is formed only in the reduced environment of tumor cells, it should not inhibit the other CA isoforms highly abundant in noncancer tissues.

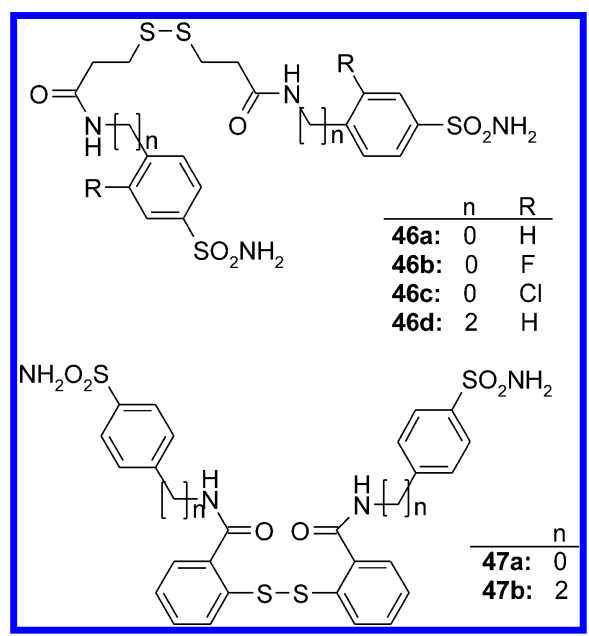

$N, N^{\prime}$-(2,2'-Dithio-dibenzoyl)bis-sulfonamide 47a is the member of this class of bioreductive compounds better characterized both from a functional and structural point of view. ${ }^{185}$ In its oxidized form this molecule is a very weak CA inhibitor with $\mathrm{K}_{\mathrm{I}} \mathrm{s}$ values in the range of $653-4975 \mathrm{nM}$ against CA I, CA II and CA IX. ${ }^{185}$ However, when reduced to the corresponding thiol derivative 48 (see Scheme 3), it becomes a good CA I inhibitor $\left(K_{I}\right.$ of $\left.276 \mathrm{nM}\right)$, and an optimal CA II and IX inhibitor, with inhibition constants in the low nanomolar range (16 nM and $9.1 \mathrm{nM}$ for CA II and CA IX, respectively). ${ }^{185}$ The molecular basis of the strong CA affinity of the reduced derivative 48 was obtained from the analysis of the crystallographic structure of the CA II/48 complex. ${ }^{185}$ This structure showed that, beyond the classical interactions of the sulfonamide anchoring group, a large number of hydrophobic interactions stabilize the organic scaffold of the inhibitor in the CA active site. In particular, the aminocarbonylbenzenesulfonamide moiety establishes strong van der Waals interactions with residues Asn67, Gln92, Val121, Phe131, Leu198, Thr199, Thr200, Pro201, and Pro202 (Figure 25A), while the aromatic

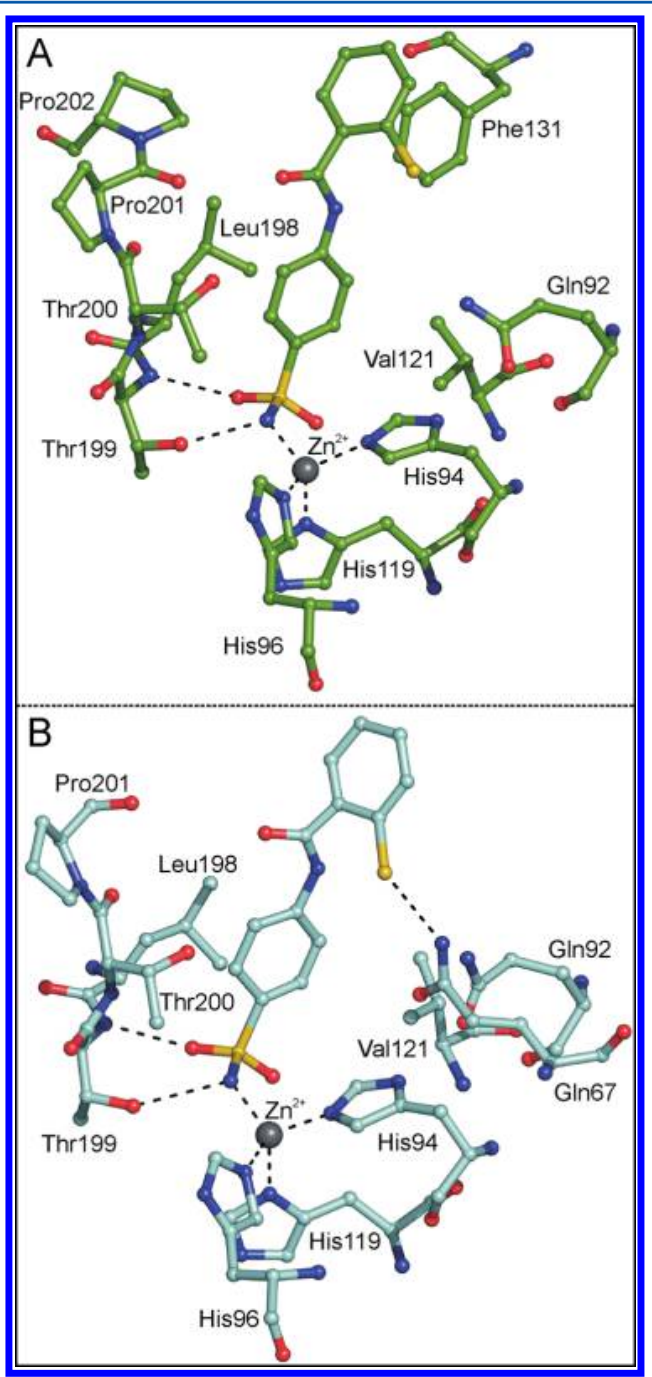

Figure 25. (A) Representation of $\mathbf{4 8}$ binding to (A) hCA II (PDB code 2 HD6) and (B) hCA IX (obtained by modeling studies). $\mathrm{Zn}^{2+}$ coordination, hydrogen bonds and residues participating in inhibitor recognition are also shown.

thiol substituent interacts poorly with the enzyme, as confirmed by its rather disordered electron density. ${ }^{185}$ Molecular modeling studies on the corresponding adduct with CA IX have revealed that the higher affinity of the molecule for CA IX with respect to CA II could be explained thanks to an additional polar interaction between enzyme residue Gln67 and the thiol group of the inhibitor (Figure 25B). ${ }^{185}$ In vivo experiments on some representative elements of such class of compounds are currently under way to test their antitumor activity. 
4.4.2. Thiophene, Thiadiazole, and Thiadiazoline Derivatives. Heterocyclic sulfonamides are among the most studied CAIs. The interest in this class of molecules started in 1945 when the pioneering studies of Davenport reported that thiophene-2-sulfonamide 49 was 40 times more active than sulfanilamide 5 as CAI. ${ }^{188}$ Starting from this observation a large number of sulfonamides incorporating a range of heterocyclic rings (such as alkyl and aryl imidazoles, benzimidazoles, benzothiazole, thiazole, pyrazine, etc.) was reported and tested for CA inhibitory properties. ${ }^{189,190}$ These studies allowed to derive the following general considerations: (i) sulfonamides containing five-membered rings were usually more efficient as CAIs than those containing six-membered rings; (ii) the presence of nitrogen and sulfur atoms within the ring was associated to better CA inhibitory properties. Indeed, 5substituted-1,3,4-thiadiazole-2-sulfonamide 50, 6-substitutedbenzothiazole-2-sulfonamide $\mathbf{5 1}$, and 1,3,4-thiadiazoline-2sulfonamides $\mathbf{5 2}$ were demonstrated to act as particularly efficient inhibitors. Good inhibitory properties were also shown by derivatives of thiophene-2-sulfonamide $\mathbf{5 3}$ with different substitution patterns. ${ }^{191}$ Finally, sulfonamide derivatives incorporating the bicyclic thieno-thiopyran ring (54 and 55), which were used as lead compounds for the development of the first clinically used, topically acting antiglaucoma sulfonamides, dorzolamide 56 and brinzolamide $\mathbf{5 7}$, behaved as effective CAIs against a range of isoforms, among which CA II, IX, XII, etc. $^{2,13,192}$

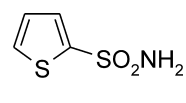

49

Thiadiazole, thiadiazoline and thiophene derivatives of type (50, 52, and 53) are among the most structurally studied heterocyclic sulfonamides. Indeed, a very large number of crystallographic structures of adducts of such molecules with hCA II has been reported. ${ }^{193-197}$ These studies showed that, independently on the nature of the $\mathrm{R}$ substituent, the heterocyclic sulfonamide moieties of these inhibitors are rather well superimposable when bound to the enzyme active site, with only very small differences in the ring orientation. Figure 26 summarizes the interactions that such inhibitors can establish within the hCA II active site. It can be seen that in addition to the canonical $\mathrm{Zn}^{2+}$ coordination of the sulfamoyl moiety, the organic scaffold of all these molecules establishes van der Waals interactions with residues Gln92, Val121,
Leu198, Thr199, and Thr200. ${ }^{193-197}$ Moreover, the thiadiazole ring, differently from the thiophene and thiadiazoline scaffolds, can also participate to a hydrogen bond interaction with the Thr200OG1 atom (Figure 26B). ${ }^{193,195-197}$ Varying the nature of the $\mathrm{R}$ group in thiophene, thiadiazole, and thiadiazoline derivatives (see as examples compounds 58-69), a large number of compounds was obtained, which significantly differ in their affinities for various CA isoforms, and in their physicochemical properties. ${ }^{118,189-191,196,198-203}$ As an example, acetazolamide 58, one of the most studied CAIs, has been obtained by the derivatization of the exocyclic amino moiety substituting C5 of the 1,3,4-thiadiazole ring with an acetyl group. ${ }^{193,204,205}$ This compound, which was shown to possess very good pharmacological properties, after its discovery in 1954, has been widely used for the treatment of edema, glaucoma, gastroduodenal ulcers, mountain sickness, and many other neuromuscular disorders. ${ }^{204,205}$

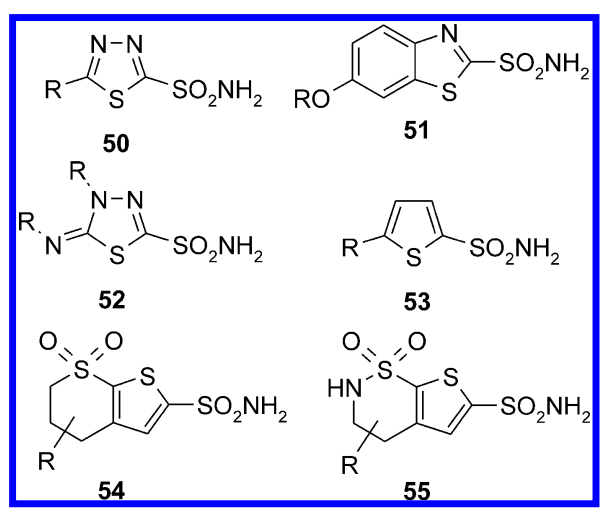

Among the thiadiazoline derivatives one should mention methazolamide 61, a compound that has been, and it is still used, as antiglaucoma drug for more than 40 years. ${ }^{6}$ Thanks to its excellent pharmacological properties, it has been also utilized as lead compound for the design of many CAIs. ${ }^{146,198,200,202}$ Among these, derivatives incorporating fluorinated tails (see as examples compounds 62 and 63$)^{202}$ are of particular interest since, beyond showing a very strong in vitro affinity (in the low nanomolar range) for the critically relevant isozymes involved in aqueous humor secretion within the eye, that is, CA II and CA IV/XII, they possess a good water solubility and a rather high lipophilicity. ${ }^{202}$ As a consequence, they have been easily formulated as eye drops at physiological $\mathrm{pH}$ values and their in vivo efficacy, tested in animal models of glaucoma, is much

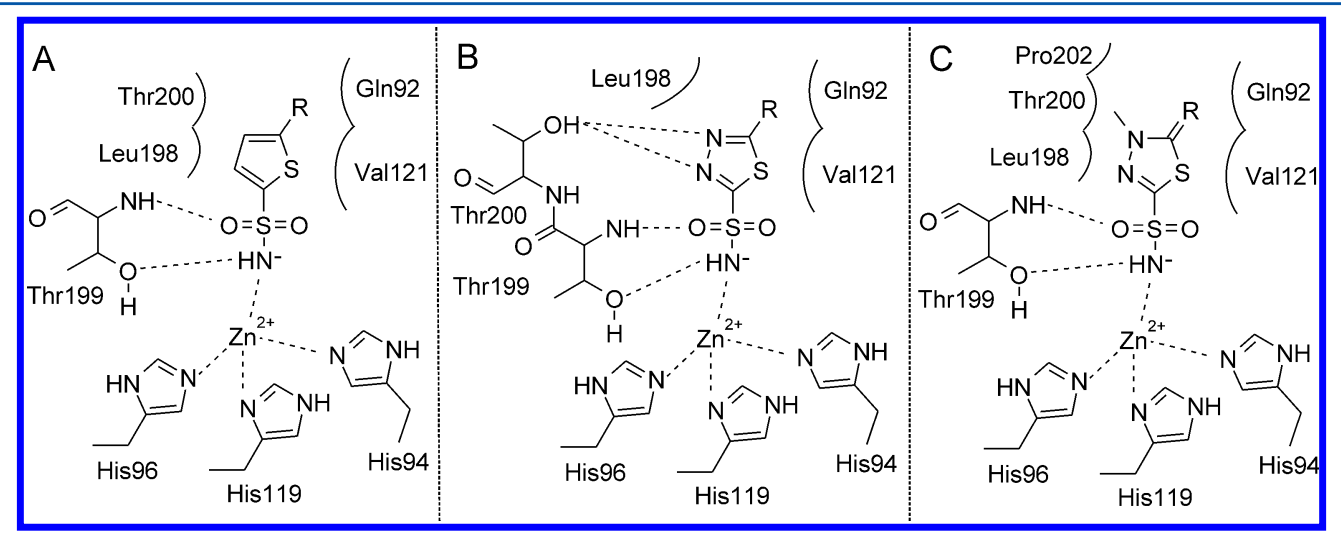

Figure 26. Schematic representation of the binding mode of (A) thiophene, (B) thiadiazole, and (C) thiadiazoline derivatives within hCA II active site. 
higher than that of the topically acting antiglaucoma agents dorzolamide 56 and brinzolamide 57.

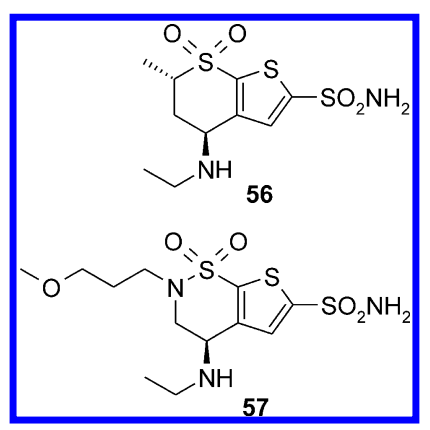

4-Methyl-5-perfluorophenylcarboximido- $\delta^{2}$-1,3,4-thiadiazoline-2-sulfonamide 63 is one of the most interesting compounds of this series. Indeed, it possesses a hCA II inhibitory activity almost 10 times higher $\left(K_{\mathrm{I}}\right.$ of $\left.1.5 \mathrm{nM}\right)$ than its precursor, methazolamide $61\left(K_{\mathrm{I}}\right.$ of $\left.14 \mathrm{nM}\right)$ and has been shown to act as an efficient topically acting antiglaucoma agent. $^{202}$ The crystallographic structure of its adduct with hCA II was utilized to derive the molecular factors that can explain the enhanced affinity of this type of fluorinated compounds for the CA active site. In particular, the analysis of the structure showed that the binding mode of 63 to hCA II maintains the same features of other heterocyclic sulfonamides for what concerns the thiadiazolinesulfonamide moiety, but differs when the perfluorobenzoylimino fragment is considered. Indeed, this tail makes a hydrogen bond with the side chain of residue Gln92 and a strong face-to-face stacking interaction with Phe131 (Figure 27). ${ }^{194}$

The derivatization of the $\mathrm{C} 5$ atom of the thiophene-2sulfonamide or 1,3,4-thiadiazole-2-sulfonamide scaffolds with a tail containing a second sulfonamide moiety, generating compounds of type 60 and $65-69,{ }^{195-197}$ has also been widely used to obtain CAIs with improved physicochemical properties. ${ }^{196,200}$ Benzolamide 65 is an interesting example of this

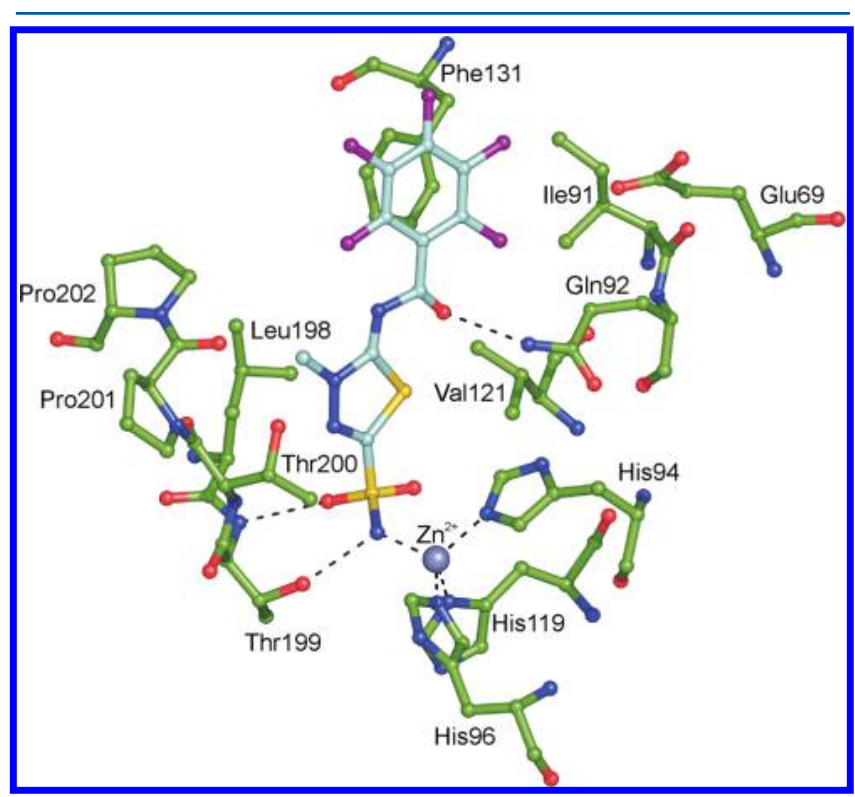

Figure 27. Active site of hCA II/63 complex showing the zinc ion coordination, hydrogen bonds and residues participating in the van der Waals interactions with the inhibitor scaffold (distance $<4.5 \AA$ ). The PDB coordinate file has been kindly provided by Claudiu T. Supuran. kind of derivatization. ${ }^{200,206}$ This molecule presents peculiar physicochemical properties. Due to the presence of the second, highly acidic sulfonamide moiety $\left(\mathrm{p} K_{\mathrm{a}}\right.$ of 3.4$),{ }^{206}$ it is much more polar than the structurally related analogue 58, and consequently crosses biological membranes much more difficultly as compared to other CAIs in clinical use. , 200,206 $^{-1}$ To modify the hydrophilic character and the lack of water/lipid solubility of 65 , some derivatives incorporating polyhalophenylsulfonamide moieties instead of phenylsulfonamide were investigated (see compound 60 as an example). ${ }^{118,202,207}$ These compounds generally retained the excellent inhibitory properties of the lead compound, but at the same time possessed much higher solubility both in water and in organic solvents, as well as better penetrability through biological membranes. ${ }^{197}$ Several structural studies on the complexes that these heterocyclic bis-sulfonamides form with hCA II are so far available. Such studies have shown that the second sulfonamide group can help to improve the binding affinity, establishing several direct or solvent-mediated polar interactions with active site residues. ${ }^{195-197}$

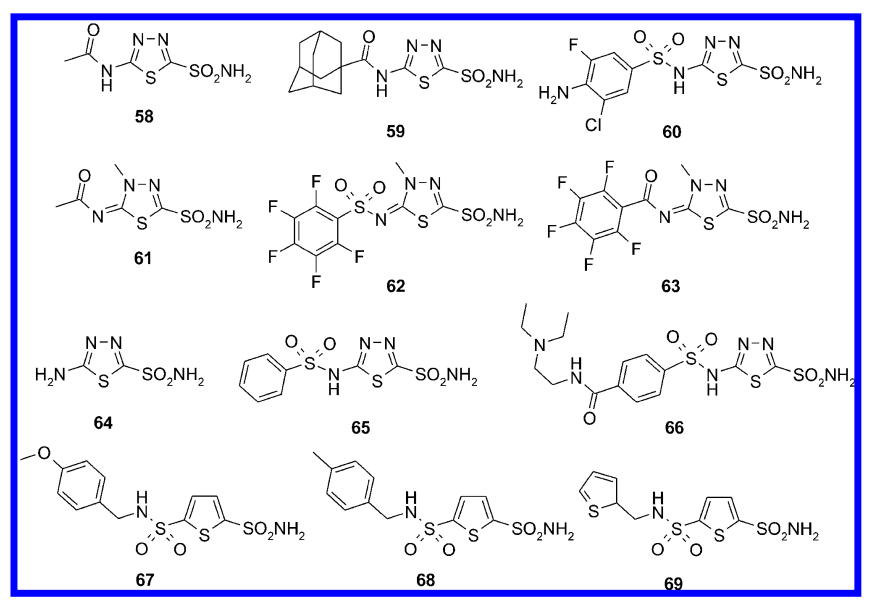

Further modifications of the thiophene disulfonamides 6769 can be obtained connecting the secondary sulfonamide group with the $\mathrm{C} 4$ atom of the thiophene ring, leading to bicyclic compounds containing the thienothiazine-6-sulfonamide-1,1-dioxide skeleton (compounds 70-79). ${ }^{196}$ The CA inhibitory properties of these molecules were investigated in detail by Boriack-Sjodin and co-workers, showing that they generally present an enhanced enzyme-inhibitor affinity with respect to the corresponding noncyclized thiophene disulfonamides. ${ }^{196}$ Structural studies suggested that the increase in binding affinity after cyclization could be ascribed to the preorganization of the secondary sulfonamide in an orientation that allows to optimize the edge-to-face interaction of its aromatic substituent with residue Phe131 (Figure 28). ${ }^{196}$ The effect of several modifications of the thienothiazine-6sulfonamide-1,1-dioxide scaffold was also investigated by means of a combined kinetic and structural approach. ${ }^{196}$ As an example, to analyze the effects of stereochemical and configurational variations at the $\mathrm{C} 4$ position of the bicyclic scaffold, kinetic and structural investigations were conducted on compounds 70-72. These studies showed that the addition of a methylamino group with $R$ stereochemistry (compound 70) slightly changes the orientation of the thiophene ring with respect to the compound without the C4 substituent (71) (Figure 29). This change does not lead, however, to dramatic consequences on the binding affinities of these compounds for 


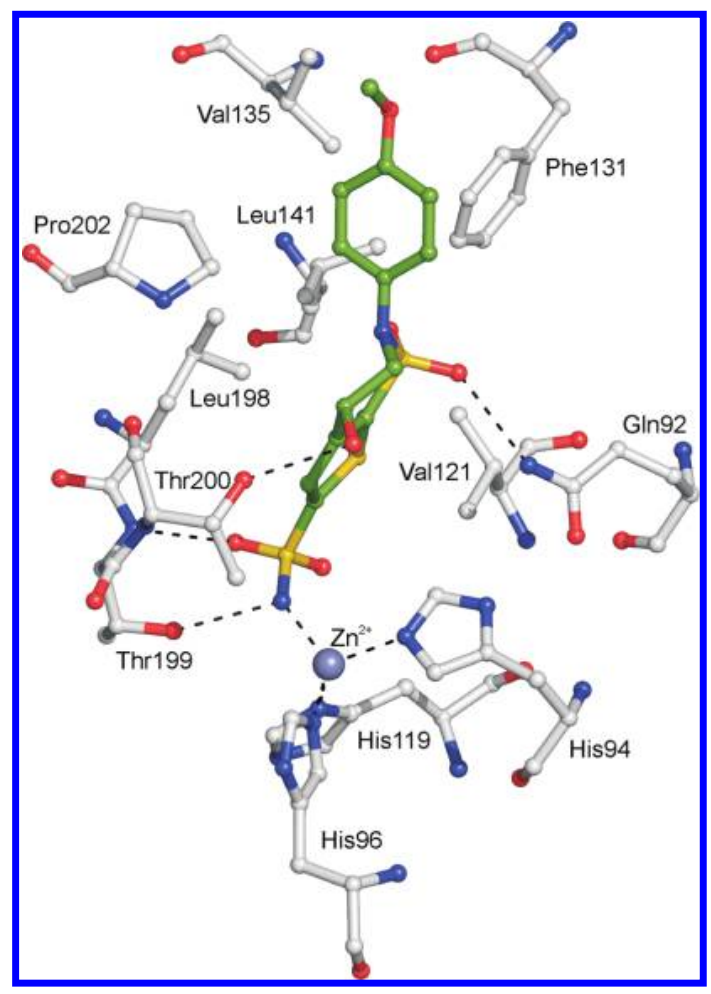

Figure 28. View of the binding of 73 to hCA II active site (PDB code 1BNT). $\mathrm{Zn}^{2+}$ coordination, hydrogen bonds and hydrophobic interactions of the inhibitor molecule (distance $<4.5 \AA$ ) are also shown.

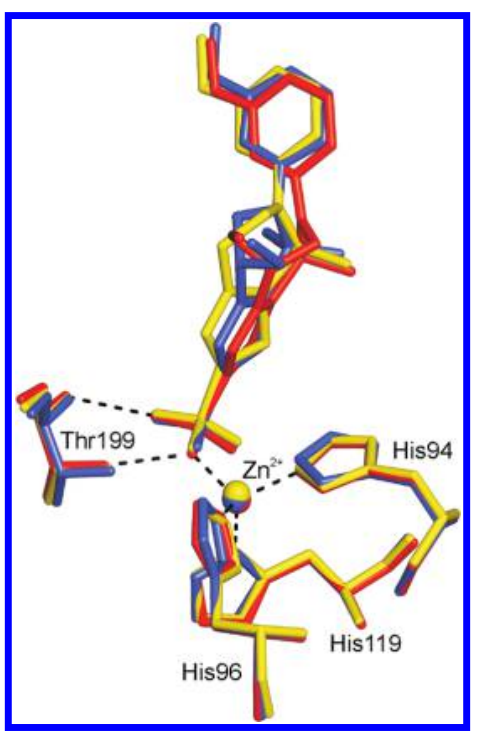

Figure 29. Superposition of hCA II/inhibitor adducts: 70 is reported in blue (PDB code 1BNM), 71 in yellow (PDB code 1BNN), and 72 in red (PDB code 1BNV). The figure shows the variable orientation of the thienothiazine-6-sulfonamide-1,1-dioxide scaffold resulting from substitutions at the inhibitor $\mathrm{C} 4$ atom.

the enzyme. On the contrary, the introduction of a methylamino moiety with $S$ stereochemistry (compound 72) causes a highly significant change in the orientation of the ring (see Figure 29) and determines a dramatic reduction in CA II binding affinity. These studies clearly indicate that thienothiazine-6-sulfonamide-1,1-dioxide derivatives having substituents with $S$ stereochemistry at $\mathrm{C} 4$ cannot be easily accommodated in the CA II active site. In agreement with this observation, when a racemic mixture of the inhibitor is used, only the $R$ enantiomer binds to the enzyme. ${ }^{196}$ The insertion of an additional degree of unsaturation between C3 and C4 in the six-membered thiazine ring, as observed in compound $\mathbf{7 5}$, does not alter the binding mode of the inhibitor. ${ }^{196}$ On the contrary, the introduction on this skeleton of a substituent in position 3 , as observed in compounds $\mathbf{7 6}$ and $\mathbf{7 7}$, has a striking effect on the inhibitor binding mode. ${ }^{208}$ In particular, the orientation of the aromatic substituents on the endosulfonamide nitrogen inside the active site changes dramatically. In fact, in the crystal structure of the hCA II/76 complex, the morpholino group is associated with the hydrophobic patch defined by Phe131, Val135, Leu198, Pro202, and Leu204, which is instead occupied by the aromatic substituent on the endosulfonamide nitrogen in the absence of a C3 substituent (Figure 30).

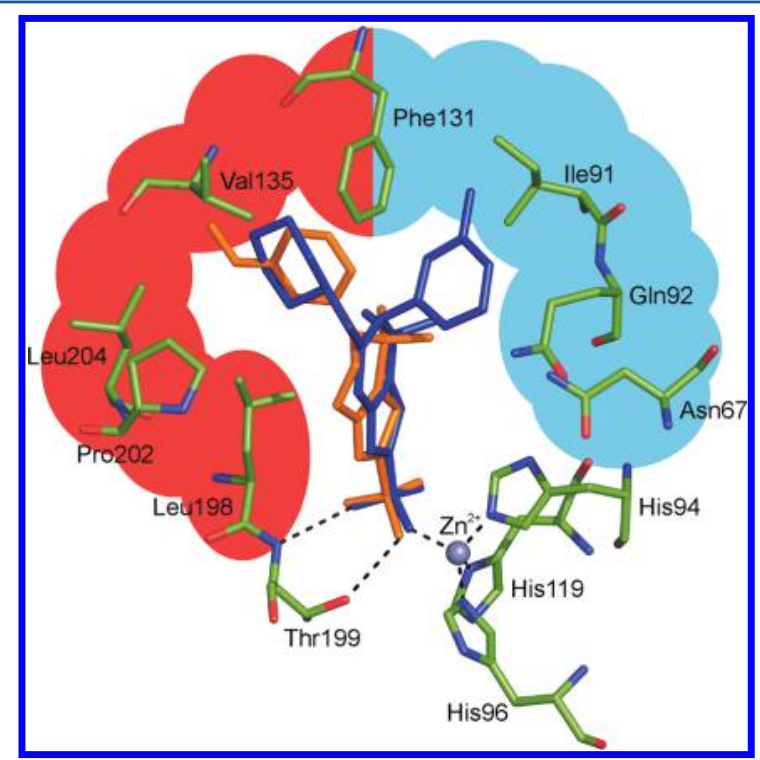

Figure 30. Structural superposition between hCA II/75 (orange, PDB code 1BN3) and hCA II/76 (blue, PDB code 1I91), showing the different orientations of the substituents on the endosulfonamide nitrogen.

Consequently, this latter is oriented toward the opposite side of the active site, interacting with residues Asn67, Ile91, Gln92, and Phe131 (Figure 30). ${ }^{208}$ The effect of the substitution of the aromatic substituents on the endosulfonamide nitrogen with aliphatic ethers, such as in $\mathbf{7 8}, \mathbf{7 9}$, and brinzolamide $\mathbf{5 7}$, was also carefully examined. ${ }^{196,208}$ These studies revealed that the loss of the edge-to-face interaction between the aromatic substituent and Phel31 had a minor impact on enzymeinhibitor affinity, as long as the aliphatic substituent group was sufficiently long to desolvate a correspondingly large hydrophobic patch in the enzyme active site.

The thienothiopyran ring is another bicyclic scaffold derived from thiophene, which has been widely investigated for the development of potent CAIs. Representative examples of CAIs containing this scaffold are the topically acting antiglaucoma drug dorzolamide 56 and its strictly related analogues 80-81, which differ from each other only in the length of the substituent on the 4-amino group: 80, proton, 81, methyl and 56, ethyl. The binding affinity of these molecules for hCA II, as well as the three-dimensional structures of the corresponding complexes, were investigated ${ }^{209}$ allowing to derive some 


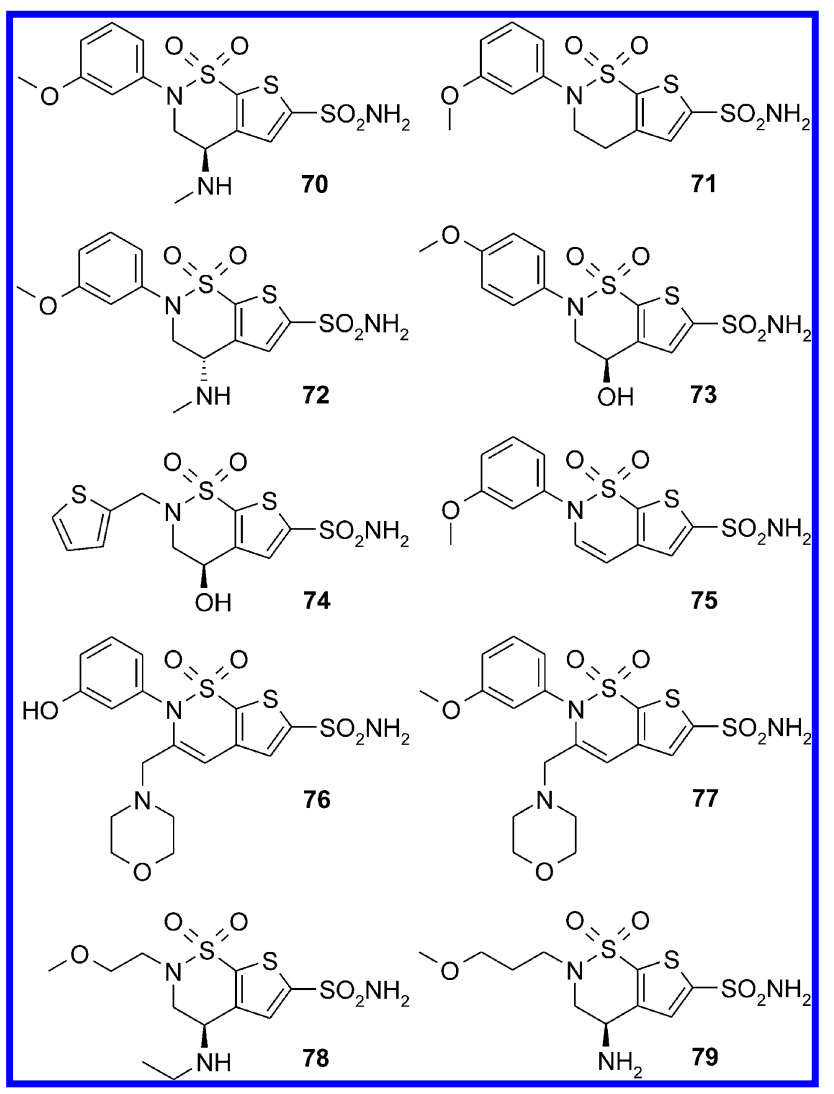

important structure-function relationships for this subclass of compounds. In particular, kinetic studies showed that $\mathbf{8 0}$ and 81 present identical hCA II binding constants (within the experimental error), whereas 56, despite the high similarity in chemical structure, shows a binding affinity 4-5 times higher. ${ }^{210}$ These findings have been well rationalized by solving the crystallographic structures of the corresponding complexes. Indeed, the analysis of such structures showed that 56, 80 and $\mathbf{8 1}$ adopt a very similar conformation and orientation within the hCA II active site. The only differences observed in these complexes concern the position of the His64 side chain. In particular, in the hCA II/80 and hCA II/81 adducts, the His64 side chain is observed in its in conformation, ${ }^{209}$ while in the hCA II/56 complex it is forced in its out conformation by the presence of the bulkier 4-aminoethyl group, with the consequent release of a water molecule to the bulk solvent. ${ }^{209}$ These findings suggest that the increase in entropy, caused by the release of the bound water molecule, plays a key role in determining higher hCA II affinity of $\mathbf{5 6}$ with respect to 80 and 81, and open new perspectives on the factors that should be considered in the design of even more potent CAIs.

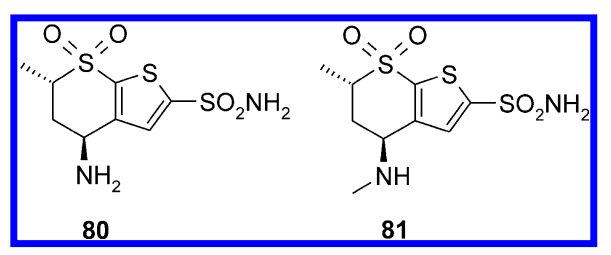

Finally, it should be also mentioned that the dorzolamide scaffold has also been recently used for the design of new bifunctional compounds of type 82-86, which beyond to act as CAIs, contain a nitric oxide (NO)-releasing moiety. ${ }^{211} \mathrm{NO}$ donors are a relatively underexplored class in the field of ocular drugs, despite the presence of NO target in all eye compartments. $^{212}$ It has been observed that hypertensive glaucoma patients have a decreased NO/cGMP content in the aqueous humor, ${ }^{213}$ moreover some NO donors have been shown to decrease intraocular pressure in normal and pathological conditions. ${ }^{214-216}$ For this reason the development of NO-donating CAIs for topical administration represents an interesting alternative to the classical antiglaucoma drugs. ${ }^{211}$ Accordingly, in vivo experiments showed that compounds 82 and $\mathbf{8 3}$ were able to induce potent intraocular pressure lowering effects in normotensive rabbits, anticipating their potential use for the treatment of glaucoma. ${ }^{211}$

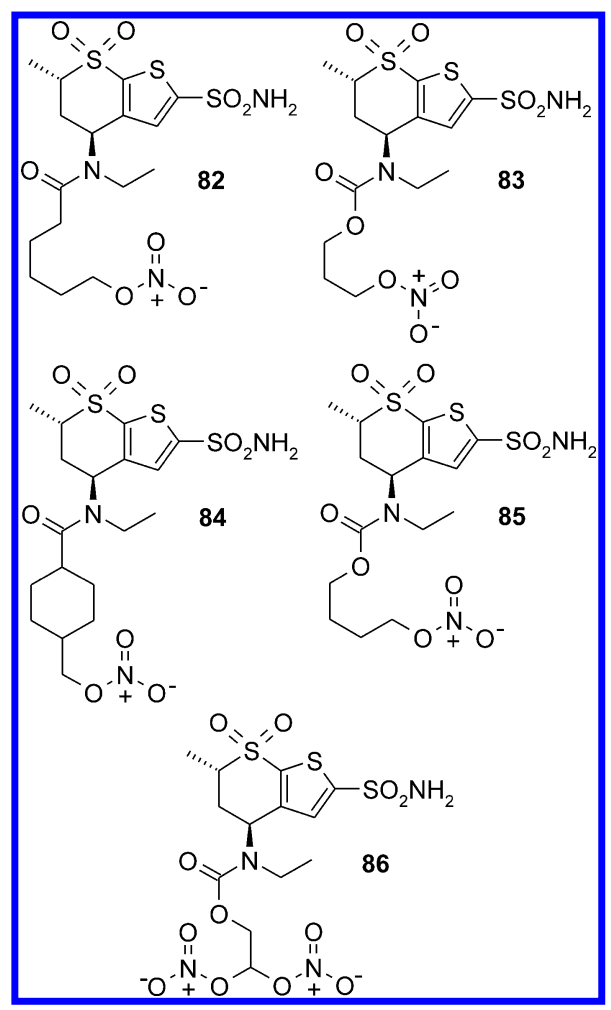

4.4.3. Sulfonamides Containing Other Ring Systems. Several other annulated bicyclic sulfonamides, different from those derived from thiophene, described in the previous paragraph, have been investigated as CAIs. Many of these derivatives, such as indanesulfonamides 87 and 88 , the thiazolinone derivative 89 , indolesulfonamide 90 , the thiabendazole derivative 91 and the 1,2,3,4-tetrahydroisoquinoline derivative 92 , have been also structurally characterized in their adducts with hCA II. ${ }^{217-222}$ The indolesulfonamide 90 is a particularly interesting representative of this type of compounds due to its peculiar CA inhibitory properties. ${ }^{219}$ Indeed, CA inhibition assays against all human isoforms showed that this molecule is a medium potency inhibitor of CA VII, IX, XII, and XIV ( $K_{\mathrm{I}}$ values in the range of $48-110 \mathrm{nM}$ ), whereas it strongly inhibits hCA I and II ( $K_{\mathrm{I}}$ values of 7.5 and $7.2 \mathrm{nM}$ for hCA I and II, respectively), ${ }^{219}$ thus demonstrating a slight selectivity for hCA I and hCA II with respect to the other isoforms. The selectivity for hCA I is a particularly interesting feature, considering that only few potent hCA I inhibitors have been reported so far ${ }^{13,14,16,204}$ and in view of the recent observations of Feener's group, which reported that substantial levels of hCA I were identified in the vitreous of patients with diabetic retinopathy. Thus, the inhibition of this isozyme could provide new opportunities for the treatment of this pathological condition. $^{28}$ The unusual inhibition profile of 90 , combined 
with its peculiar chemical structure, characterized by the presence of a hydrazide moiety in position 2 and a phenyl substituent in position 3 , suggested the possibility to utilize this molecule as lead compound for the design of isoform selective CAIs. For this aim the crystallographic structure of the adduct of 90 with hCA II was solved, offering additional hints for a structure based drug design. ${ }^{219}$ The analysis of this structure showed that the sulfonamide group classically binds to the zinc ion, the indolesulfonamide scaffold fills the bottom of the active site establishing a large number of hydrophobic interactions, while the hydrazide moiety participates in a network of hydrogen bonds with residues Asn62 and Asn67 (Figure 31A).

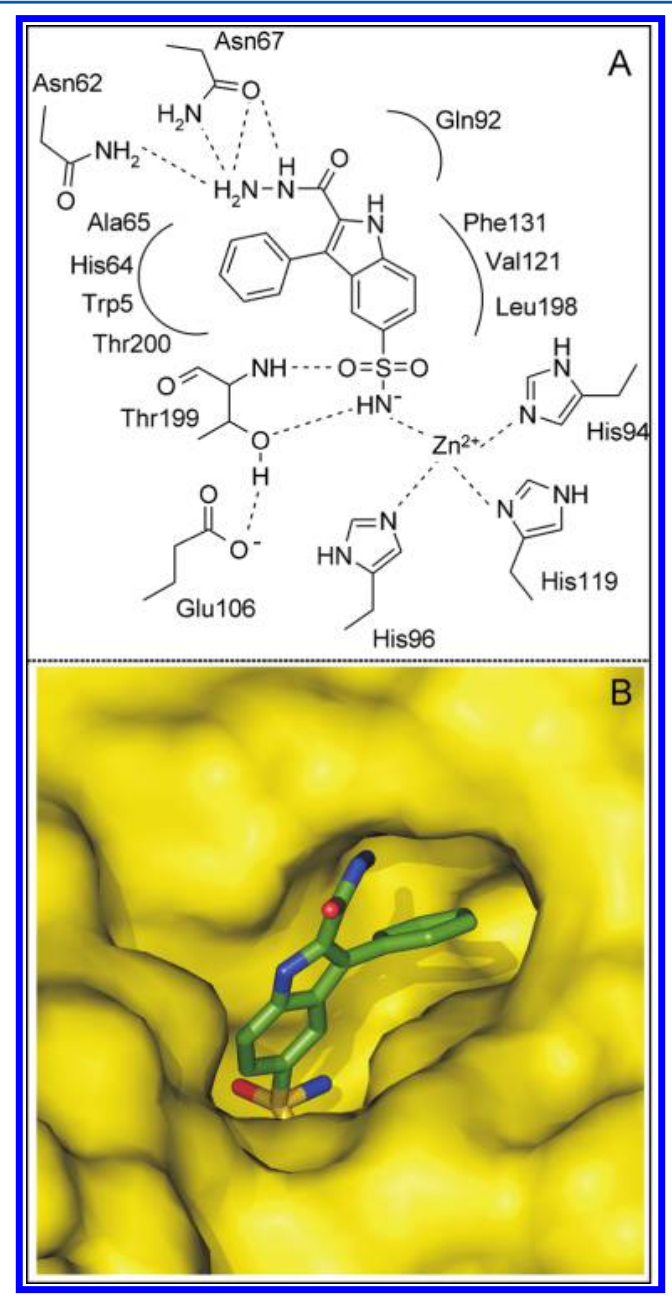

Figure 31. (A) Schematic representation of hCA II/90 (PDB entry 3B4F) active site. (B) Solvent accessible surface of hCA II showing the inhibitor 90 accommodated within the active site channel.

However, the most interesting data from the drug design point of view was the observation that the 3-phenyl group of 90 is accommodated in a sort of pocket within the active site, where enough additional space is available for various moieties to be introduced as substituents to the phenyl ring (Figure 31B). Thus using this X-ray crystal structure as starting point, the design and synthesis of a series of 2-(hydrazinocarbonyl-3substituted-phenyl)-1H-indole-5-sulfonamides, of type 93, were reported. ${ }^{23}$ Kinetic experiments performed against all the catalytically active CA isozymes showed that these molecules presented kinetic properties different from those of their lead compound 90. Indeed, they were not anymore selective for
hCA I, but presented excellent, low-nanomolar inhibition of hCA II and hCA XIV, ${ }^{223}$ showing that the substitution of the 3phenyl moiety can have dramatic consequences on the inhibition profile of such molecules. Altogether these studies indicated the indolesulfonamide scaffold as an interesting alternative to the classical benzenesulfonamides for obtaining CAIs, whose properties can be efficiently modulated by changing the nature of the substituent in the 3-position of the indole ring. ${ }^{223}$

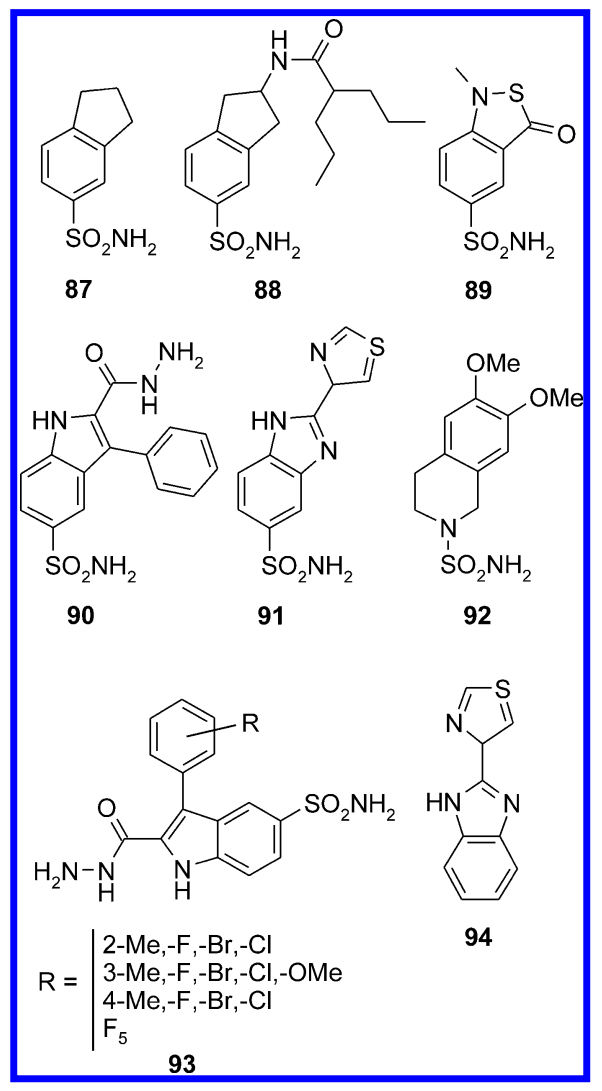

The thiabendazole molecule 94, a broad-spectrum antihelmintic drug used both for the treatment of parasitic infections in animals and humans and as an agricultural fungicide, ${ }^{224,225}$ possesses a byciclic scaffold structurally related to that of 90. Few data are at present available on the mechanism of action of this molecule, which seems to be correlated to its selective binding to the parasite $\beta$-tubulin, with subsequent prevention of microtubule formation. ${ }^{226}$ Given that the widespread resistance to many clinically used drugs for the treatment of helminthic infections represents a global health problem, it is very important to design such new pharmacological agents. In this scenario, the parasitic CAs could represent a new target for the development of a novel clinical strategy. With this aim, a thiabendazole derivative incorporating a sulfonamide functionality (compound 91) was synthesized. ${ }^{220}$ On the basis of the design hypothesis, this molecule, besides the original antihelminthic activity, had to possess the ability to bind to the CA active site, because of the presence of the sulfonamide group. Indeed, inhibition assays performed both on the CAH-4b, a CA isozyme isolated from the worm Caenorhabditis elegans, and on the mammalian CA I-XIV, indicated that this derivative strongly inhibited CAH-4b $\left(K_{\mathrm{I}}\right.$ value of $9.35 \mathrm{nM}$ ) and also acted as potent inhibitor of isoforms hCA I, IV, VB, VI, IX, and XII ( $K_{\mathrm{I}}$ values in the range of 6.4$50.9 \mathrm{nM}){ }^{220}$ The binding mechanism of 91 to the CA active 
site was elucidated by the analysis of its X-ray structure in complex with hCA II, showing that 91 presents the canonical coordination of the $\mathrm{Zn}^{2+}$ ion by means of the sulfonamide moiety, with the benzimidazole scaffold filling the active site channel of the enzyme and participating in several hydrophobic contacts with the enzyme and into a $\pi-\pi$ stacking interaction with Phe131. Interestingly, the comparative analysis of this thiabendazole derivative with compound 90 reveals that the arrangement of their indole/benzimidazole moieties within hCA II active site is completely different, despite the high similarity of their chemical structure. Indeed, the bicyclic scaffolds of the two inhibitors are tilted by almost $90^{\circ}$ with respect to each other (Figure 32). This different orientation

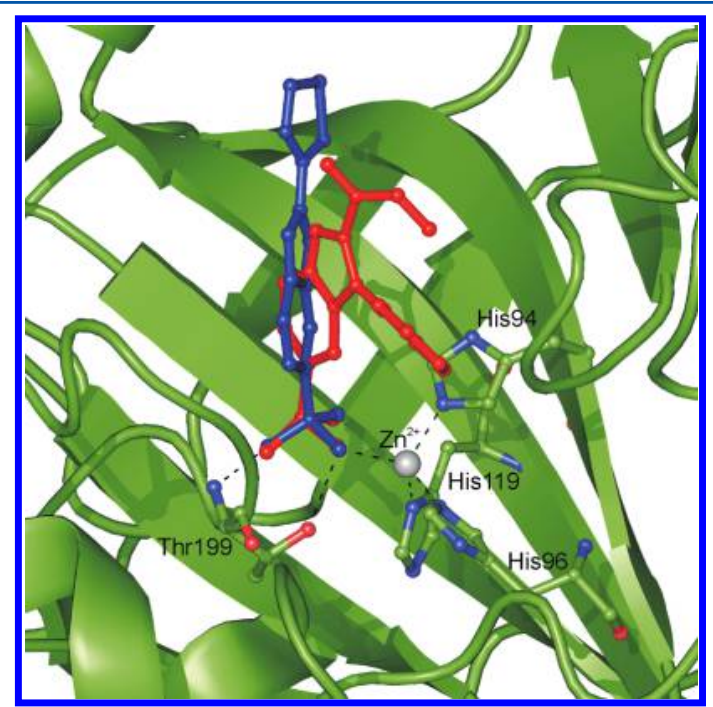

Figure 32. Superposition of the hCA II/inhibitor adducts: 90 is reported in red (PDB entry 3B4F) and 91 in blue (PDB entry 3FFP). $\mathrm{Zn}^{2+}$ coordination and hydrogen bonds of the sulfonamide moiety are also shown. The enzyme is represented as ribbon diagram.

could be ascribed to the different substitution pattern of these two bicyclic systems, highlighting once again that the nature of the substituents on such scaffolds can significantly modulate the binding mode to the enzyme and consequently the binding affinity. ${ }^{220}$

4.4.4. Thiazide Diuretics. Thiazide diuretics represent a class of pharmacological agents widely used for the treatment of hypertension, which exert their action by promoting sodium chloride (and osmotically obligated water) excretion in different segments of nephron. ${ }^{229}$ They have been the first well-tolerated antihypertensive drugs that significantly reduced cardiovascular disease morbidity and mortality in placebocontrolled clinical studies, ${ }^{227-234}$ and still represent an important therapeutic option in patients with cardiovascular diseases, but also for those suffering type II diabetes, obesity, and related metabolic complications. $227,229-234$<smiles>C1=Nc2ccccc2SN1</smiles>

95

This class of molecules includes derivatives of benzothiadiazine 95, but also compounds which present a similar pharmacological action, but do not incorporate the thiazide scaffold. The latter compounds are properly termed thiazidelike diuretics. Trichloromethiazide 96, chlorthalidone 97,

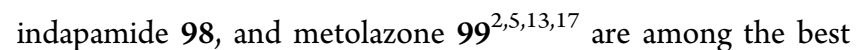
known members of this class of agents. These compounds share several structural features, such as the presence of a $\mathrm{SO}_{2} \mathrm{NH}_{2}$ moiety attached to a chloro-benzene scaffold, with the halogen atom in ortho to the sulfamoyl group, as well as various bulky groups substituting the aromatic scaffold. Right from the start the presence of a primary $\mathrm{SO}_{2} \mathrm{NH}_{2}$ group suggested that these compounds could also act as CAIs; however, when they were first launched, in 60 s or 70 s, only CA isozymes I and II were known. This is the reason for which the interaction of such diuretics with all catalytically active CA isoforms has been only recently investigated. ${ }^{235-238}$ The corresponding inhibition data, obtained by means of a stopped-flow assay, showed that these compounds behave as weak inhibitors of isozyme II, usually in the micromolar range, ${ }^{235-238}$ while they considerably inhibit other isozymes, known nowadays to be involved in critical physiological processes (see Table 3). Crystallographic studies also confirmed the interaction of this class of compounds with the CA active site. In particular, the X-ray structures of the adducts of three of these thiazide diuretics (96-98) with hCA II were reported, ${ }^{235-237,239}$ showing that these inhibitors present a rather similar binding mode to the enzyme active site (Figure 33A,B,C). Indeed, in all complexes the tetrahedral geometry of the zinc ion and the hydrogen bond network involving the inhibitor sulfonamide moiety and amino acid residues from the enzyme active site were observed. ${ }^{53,235-239}$ The sulfamoyl-chlorophenyl fragments of these three sulfonamides were well superimposable with each other (see Figure 33D), making several favorable van der Waals interactions. However, significant differences, which could explain the different binding affinity toward CA II of these structurally related compounds, were observed in the orientation of the remaining part of the 96-98 scaffolds. As a consequence of these different orientations, the tails of 96 and 97 were stabilized by several hydrogen bond interactions (Figure $33 \mathrm{~A}, \mathrm{~B})$, while the tail of $\mathbf{9 8}$ was stabilized only by van der Waals contacts (Figure 33C). ${ }^{235-239}$

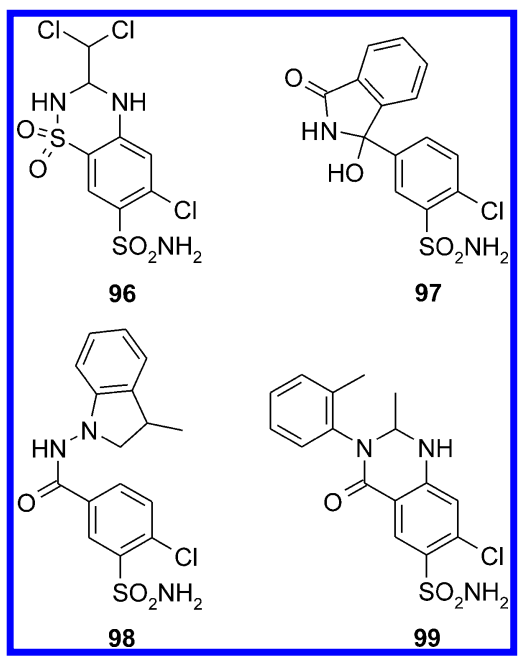

Altogether these kinetic and structural studies demonstrated the capability of thiazide diuretics to efficiently interact with the active site of several CA isoforms. This is a rather interesting result considering the wide clinical use of such compounds. Indeed, it is tempting to speculate that the inhibition of the CAs, present in blood vessels or in the kidneys, could be responsible in some way for additional therapeutic effects of these drugs or for some of their side effects, such as the recently 
Table 3. Inhibition Data of the Thiazide Diuretics 96-99 against CA Isoforms I-XIV ${ }^{a}$

\begin{tabular}{|c|c|c|c|c|}
\hline \multirow[b]{2}{*}{ isozyme $^{b}$} & \multicolumn{4}{|c|}{$K_{\mathrm{I}}(\mathrm{nM})$} \\
\hline & 96 & 97 & 98 & 99 \\
\hline $\mathrm{hCA} \mathrm{I}^{c}$ & 345 & 348 & 51900 & 54000 \\
\hline $\mathrm{hCA} \mathrm{II}^{c}$ & 91 & 138 & 2520 & 2000 \\
\hline $\mathrm{hCA} \mathrm{III}^{c}$ & $5.6 \times 10^{5}$ & $1.1 \times 10^{4}$ & $2.3 \times 10^{5}$ & $6.1 \times 10^{5}$ \\
\hline hCA IV ${ }^{c}$ & 449 & 196 & 213 & 216 \\
\hline hCA VA ${ }^{c}$ & 763 & 917 & 890 & 750 \\
\hline $\mathrm{hCA} \mathrm{VB}^{c}$ & 134 & 9 & 274 & 312 \\
\hline $\mathrm{hCA} \mathrm{VI}{ }^{c}$ & 2459 & 1347 & 1606 & 1714 \\
\hline $\mathrm{hCA} \mathrm{VII}^{c}$ & 7.9 & 2.8 & 0.23 & 2.1 \\
\hline $\mathrm{hCA} \mathrm{IX}^{d}$ & 87 & 23 & 36 & 320 \\
\hline $\mathrm{hCA} \mathrm{XII}^{d}$ & 312 & 4.5 & 10 & 5.4 \\
\hline $\mathrm{mCA} \mathrm{XIII}^{c}$ & 645 & 15 & 13 & 15 \\
\hline hCA XIV & 3450 & 4130 & 4950 & 5432 \\
\hline
\end{tabular}

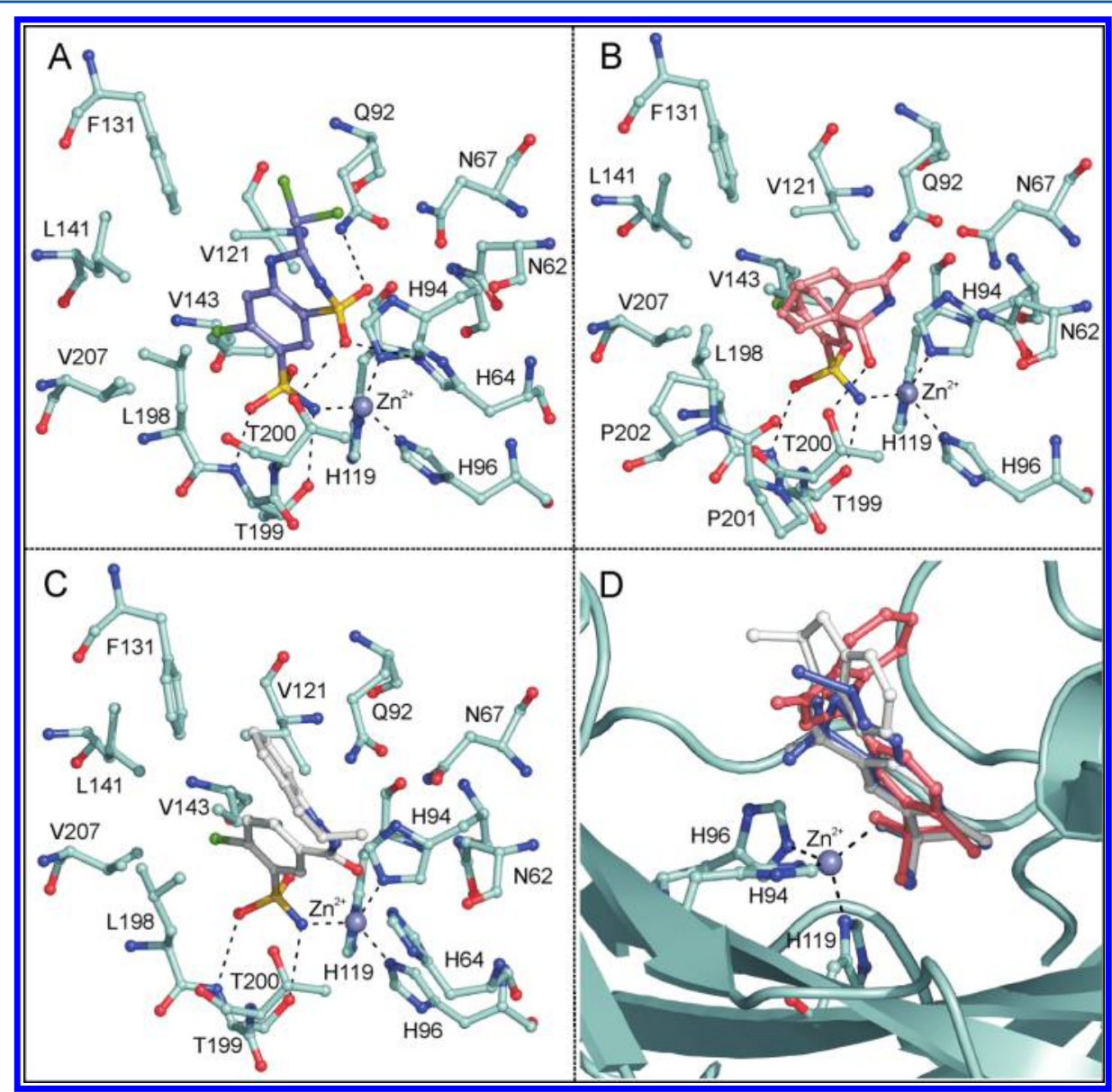

Figure 33. View of the binding mode of (A) 96 (PDB code 1ZGF), (B) 97 (PDB code 3F4X), and (C) 98 (PDB code 3BL1) to hCA II active site. $\mathrm{Zn}^{2+}$ coordination, hydrogen bonds and hydrophobic interactions (distance $<4.5 \AA$ ) stabilizing the inhibitors are also reported. (D) Superposition of hCA II-inihibitor structures: 96 is colored in blue, 97 in salmon, and 98 in white. hCA II is represented as ribbon diagram.

observed protective role of indapamide against ischemiainduced injury and dysfunction of the blood-brain barrier, ${ }^{230}$ or its organ-protective effect in animal models of renal failure. $^{233,234}$ However, no specific biochemical or pharmacological studies to prove these hypotheses are available so far. Further studies in this field could thus offer novel therapeutic applications of such compounds. It should be also mentioned the low nanomolar inhibitory action of some of these sulfonamides against isoforms involved in important patholo- gies, such as CA IX and XII (cancer and metastases) or CA VII (epilepsy). ${ }^{238}$ However, detailed in vivo studies for establishing alternative uses of these compounds are lacking for the moment.

4.4.5. Aliphatic Sulfonamides. A dogma in the CA drug design was that aliphatic sulfonamides with general formula $\mathrm{R}$ $\mathrm{SO}_{2} \mathrm{NH}_{2}\left(\mathrm{R}=\mathrm{Me}, \mathrm{PhCH}_{2}\right)$ were inactive as inhibitors, differently from the aromatic/heterocyclic ones of the type Ar- $\mathrm{SO}_{2} \mathrm{NH}_{2}{ }^{6}$ This view was subsequently challenged, ${ }^{240}$ being 
shown that aliphatic sulfonamides incorporating perhaloalkyl moieties of the type $\mathrm{C}_{n} \mathrm{X}_{2 n+1} \mathrm{SO}_{2} \mathrm{NH}_{2}(n=1-4, \mathrm{X}=\mathrm{F}, \mathrm{Cl})$ potently inhibit several $\mathrm{CA}$ isozymes. Consequently, this type of compounds has become object of great interest in the search of molecules with improved selectivity/inhibition profiles as compared to the classical aromatic/heterocyclic sulfonamide CAIs. However, only little structural information is available so far on their binding mode to the CA active site.

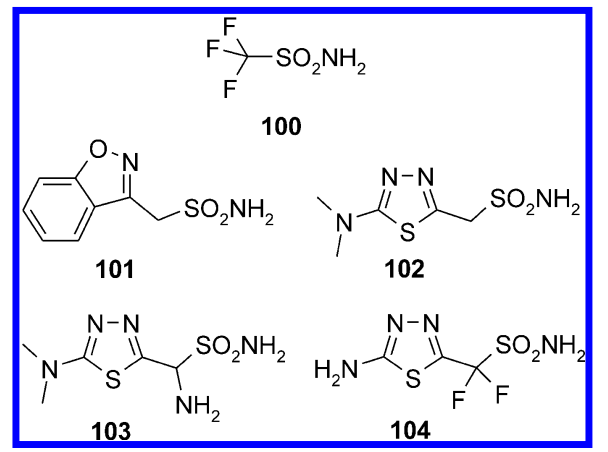

The first aliphatic sulfonamide to be structurally characterized in complex with hCA II was trifluoromethane sulfonamide 100. ${ }^{241}$ This compound showed significant CA inhibitory properties, mainly attributable to the strong acidity caused by the presence of the strongly electron withdrawing $\mathrm{CF}_{3}$ moiety in close proximity to the sulfonamide group. ${ }^{241}$ The structural analysis of the CA II/100 complex showed that the binding mode of this inhibitor to the CA active site was considerably different from that of classical aromatic/ heterocyclic sulfonamides. Indeed, differently from bulky aromatic groups of aromatic/heterocyclic sulfonamides, which generally point toward the external part of the active site cleft (Figure 34), the small hydrophobic trifluoromethyl moiety was oriented inward into the active site, making strong van der Waals contacts with residues Val121, Val143, Leu198, Thr199, and Trp209. This different orientation caused also a rearrangement of the sulfonamide group, which was rotated by about $180^{\circ}$ around the sulfur-nitrogen bond, with respect to the one present in aromatic/heterocyclic sulfonamides (Figure 34). ${ }^{240}$

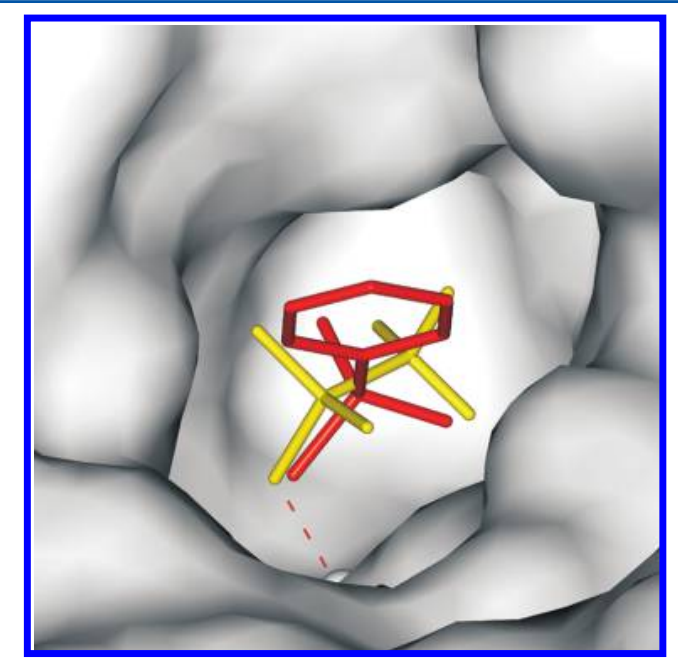

Figure 34. Structural superposition between hCA II/100 (yellow, PDB code 1BCD) and hCA II/benzenesulfonamide 6 (red, PDB code 2WEJ) showing the different orientation of the inhibitors within hCA II active site. The enzyme is represented as a surface-model and the catalytic zinc ion as a sphere.
Compounds of type 101-104, including the widely used antiepileptic drug zonisamide $101,{ }^{242-244}$ represent a different type of aliphatic sulfonamide, characterized by the presence of a heteroaromatic ring connected to the sulfonamide moiety through an aliphatic spacer. X-ray crystallographic studies of adducts of these inhibitors with hCA II highlighted that, although these compounds have a similar organic scaffold, they present significant differences in their binding mode to the enzyme (Figure 35). Indeed, while the heterocyclic rings of 101-103 are rather well superimposable, all of them being oriented toward the hydrophobic part of the active site (Figure $35 \mathrm{~A}, \mathrm{~B}, \mathrm{C}){ }^{242-244} 104$ presents a completely different orientation, being found toward the hydrophilic region, as a consequence of a $\pi$-stacking interaction with the imidazole ring of His94 (Figure 35D). ${ }^{244}$ This peculiar orientation is probably caused by the formation of a strong hydrogen bond of the $-\mathrm{CF}_{2^{-}}$linker with the Thr2000G1 atom. A further hydrogen bond interaction between the $\mathrm{NH}_{2}$ group of the inhibitor and the Asn67OD1 atom contributes to the stabilization of the adduct (Figure 35D).

Interestingly, a detailed comparison of the CA binding mode of 101-104 with structurally related heterocyclic sulfonamides, such as acetazolamide $\mathbf{5 8}$, revealed that, when bound to the enzyme, all these molecules conserve the tetrahedral geometry of the zinc ion and the hydrogen bond network between the sulfonamide moiety and the enzyme active site. ${ }^{242-244}$ Nevertheless, they present a completely different orientation of the heterocyclic ring (Figure 36). In fact, the presence of the aliphatic spacer in compounds 101-104 introduces an additional rotational freedom between the sulfonamide and the ring moiety allowing orientations inside the active site not accessible to the classical heterocyclic CAIs. These studies highlighted novel binding modes of aliphatic sulfonamides, suggesting that these compounds can represent an interesting alternative to the classical aromatic/heterocyclic sulfonamides in the development of CA isoform-selective inhibitors.

\subsection{Sulfamates and Sulfamides}

As outlined in the previous paragraphs, the presence of a zincbinding group (ZBG) in the scaffold of the CAIs directly interacting with the metal ion present within the enzyme active site is one of the main factors influencing inhibitory activity. The classical ZBG for the design of CAIs is the unsubstituted sulfonamide. $^{2,12-15,53,114}$ The most obvious bioisosteres of this group are the sulfamate and sulfamide ones, in which an additional electron-withdrawing atom/group ( $\mathrm{O}$ or $\mathrm{NH}$, respectively) is directly attached to the sulfamoyl function, generating compounds with general formula $\mathrm{R}-\mathrm{O}-\mathrm{SO}_{2} \mathrm{NH}_{2}$ or $\mathrm{R}-\mathrm{NH}-\mathrm{SO}_{2} \mathrm{NH}_{2}$. However, only relatively later after the sulfonamides started to be used clinically, ${ }^{6}$ these two groups of derivatives were considered as interesting candidates for obtaining CAIs. The first report on sulfamates as CAIs was from Maren's group ${ }^{245}$ and from Maryannof et al. ${ }^{246}$ Subsequently Supuran's group showed in $1996^{247}$ that the simple sulfamide 105 and sulfamic acid 106 bind to the metal ion in the $\mathrm{Co}^{2+}$-substituted CA II, whereas the X-ray structures of these two simple compounds in adduct with CA II have been reported several years later. ${ }^{108}$ Such structures showed that these two molecules were able to participate in a more intricate hydrogen bond network than that formed by the classical sulfonamide inhibitors, due to the presence of the additional oxygen/nitrogen atom (Figure 37). ${ }^{108}$ Nowadays there are a large number of aromatic, heterocyclic, aliphatic and sugar- 


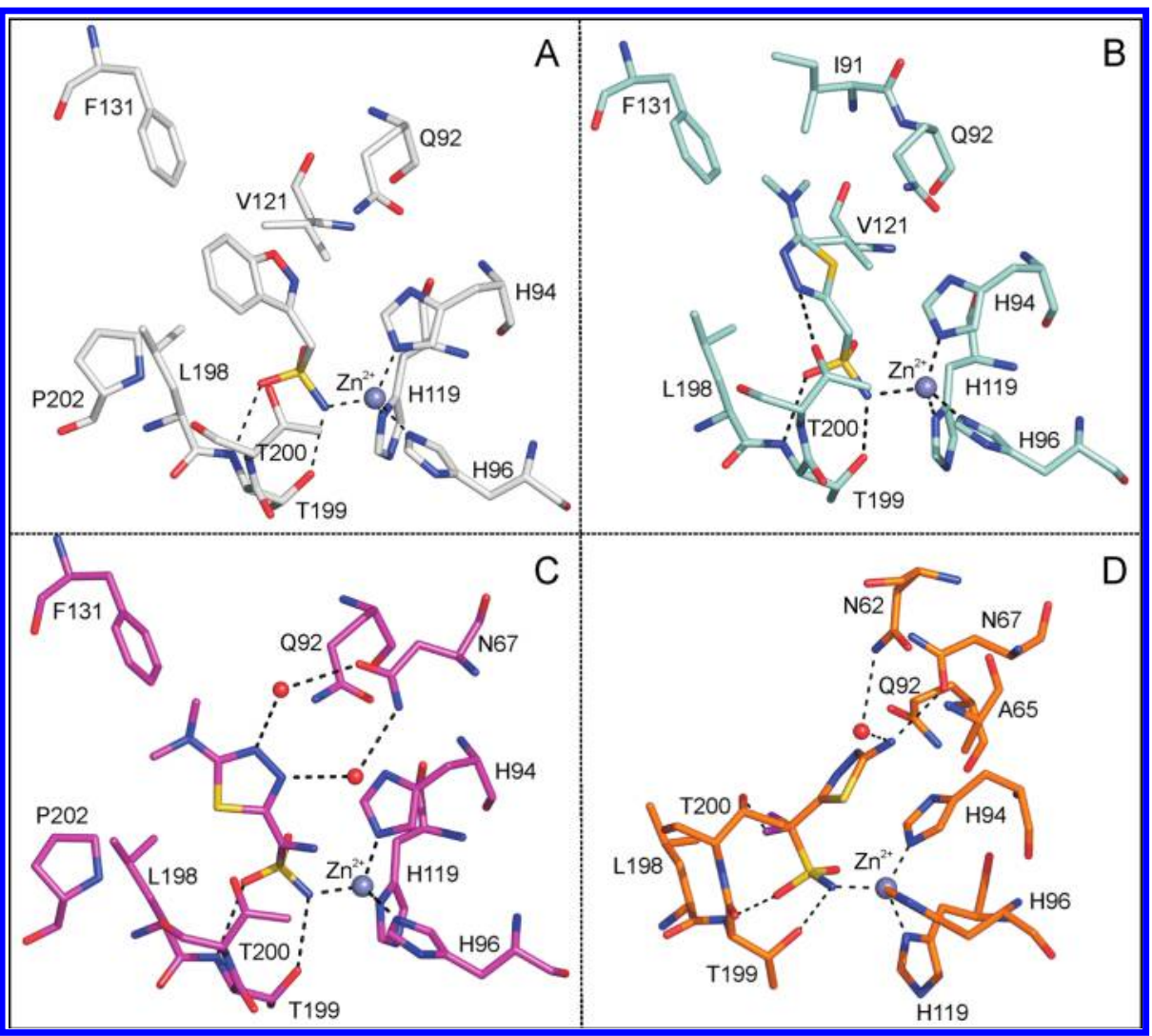

Figure 35. Active site representation of hCA II in complex with (A) 101, ${ }^{242}$ (B) 102 (PDB code 3BLO), (C) 103 (PDB code 2EU2), and (D) 104 (PDB code $2 \mathrm{EU} 3) . \mathrm{Zn}^{2+}$ coordination, hydrogen bonds and residues participating in van der Waals interactions are also shown. Water molecules are indicated as red spheres.

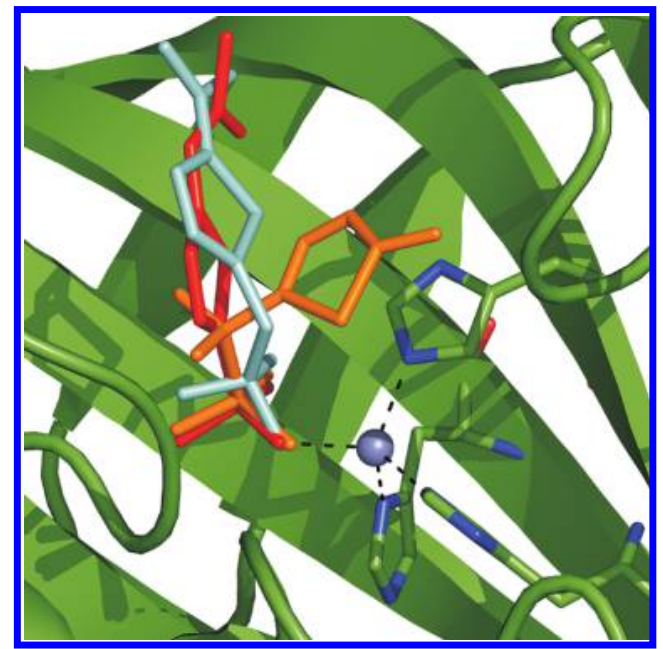

Figure 36. Superposition of hCA II/inhibitor adducts showing the different orientation of aliphatic sulfonamides from the classical heterocyclic ones. Acetazolamide $\mathbf{5 8}$ (PDB code 1ZSB) is reported in red, 102 in cyan, and 104 in orange; hCA II is represented as ribbon diagram.

based sulfamates and sulfamides which were shown to possess highly effective inhibitory properties against all known mammalian isoforms. ${ }^{2}$ Thus, sulfamates and sulfamides constitute a highly important class of CAIs, with some derivatives (i.e., topiramate) clinically used for the treatment of epilepsy and obesity. ${ }^{20-22,25,248}$

4.5.1. Aliphatic Sulfamates. Mono- and bis-aliphatic sulfamates are an interesting class of CAIs, which potently

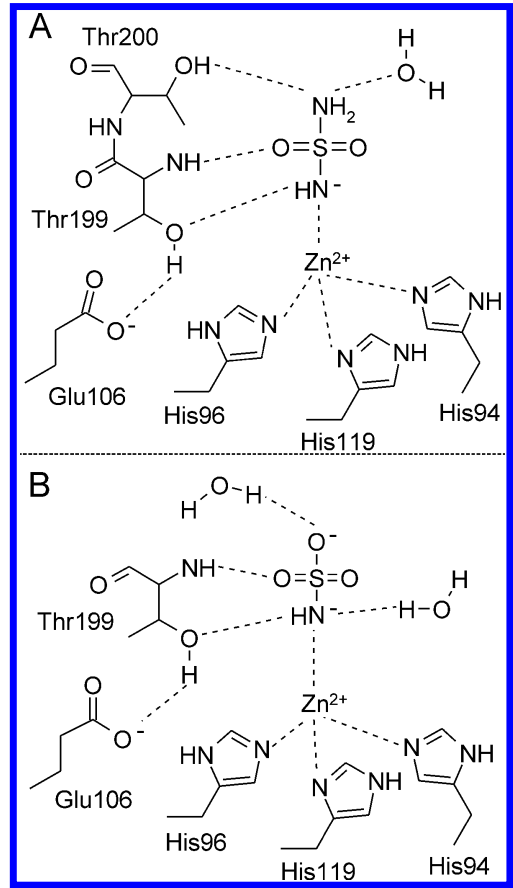

Figure 37. Schematic representation of the binding mode of (A) sulfamide 105 and (B) sulfamate 106 to hCA II active site. $\mathrm{Zn}^{2+}$ coordination and $\mathrm{H}$-bond interactions stabilizing inhibitor molecules are also reported.

inhibit several CA isozymes. Recently new compounds belonging to this class, that is, 107-110, were characterized 


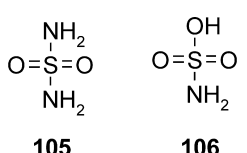

both structurally and kinetically. ${ }^{249-252}$ Kinetic studies showed that these compounds were nanomolar inhibitors of only a few isoforms, namely, hCA II, IX and XII, unlike aromatic/ heterocyclic sulfonamides in clinical use which promiscuously inhibit with low nanomolar affinity most CA isozymes (Table 4). Moreover, the bis-sulfamates showed higher affinity for the

Table 4. Inhibition Data of Sulfamates 107-110 against CA Isoforms I-XIV, by a Stopped-Flow $\mathrm{CO}_{2}$ Hydrase Assay ${ }^{a}$

\begin{tabular}{lcccc} 
& \multicolumn{5}{c}{$K_{\mathrm{I}}(\mathrm{nM})$} \\
\cline { 2 - 5 } isozyme $^{b}$ & $\mathbf{1 0 7}$ & $\mathbf{1 0 8}$ & $\mathbf{1 0 9}$ & $\mathbf{1 1 0}$ \\
$\mathrm{hCA} \mathrm{I}^{c}$ & 3.7 & 530 & 378 & 890 \\
$\mathrm{hCA} \mathrm{II}^{c}$ & 2.6 & 0.7 & 14.6 & 24.5 \\
$\mathrm{hCA} \mathrm{III}^{c}$ & 9100 & 877 & 1128 & 1206 \\
$\mathrm{hCA} \mathrm{IV}^{c}$ & 79.9 & 67.6 & 84.1 & 91.2 \\
$\mathrm{hCA} \mathrm{VA}^{c}$ & 1254 & 805 & 1202 & 1368 \\
$\mathrm{hCA} \mathrm{VB}^{c}$ & 624 & 888 & 1457 & 1592 \\
$\mathrm{hCA} \mathrm{VI}^{c}$ & 795 & 1334 & 1020 & 1414 \\
$\mathrm{hCA} \mathrm{VII}^{c}$ & 76.6 & 88.9 & 79.6 & 92.3 \\
$\mathrm{hCA} \mathrm{IX}^{d}$ & 25.0 & 23.1 & 4.0 & 7.0 \\
$\mathrm{hCA} \mathrm{XII}^{d}$ & 7.0 & 7.9 & 7.9 & 8.2 \\
mCA XIII $^{d}$ & 1125 & 956 & 569 & 960 \\
$\mathrm{hCA} \mathrm{XIV}^{c}$ & 390 & 711 & 605 & 852
\end{tabular}

${ }^{a}$ Data were obtained by ref $252 .{ }^{b} \mathrm{~h}=$ human, $\mathrm{m}=$ murine isozyme. ${ }^{c}$ Full length enzyme. ${ }^{d}$ Catalytic domain.

tumor-associated isozyme CA IX compared to hCA II, whereas the opposite was true for the corresponding monosulfamates (Table 4). Crystallographic and molecular modeling studies ${ }^{252}$ helped to rationalize this result. In particular, the X-ray crystal structures of the adducts of $\mathbf{1 0 8}$ and $\mathbf{1 1 0}$ with hCA II were solved (Figure 38A,B) and then used as template to build the models of the corresponding hCA IX complexes (Figure 38C,D). The comparison of hCA II/108 and hCA IX/108 adducts showed that while the $\mathrm{Zn}^{2+}$ binding site and the key hydrogen bonds between the sulfamate moiety of the inhibitor and the enzyme active site were preserved in both complexes, different patterns of van der Waals and/or hydrogen bond interactions, established by the inhibitor tail, were observed. Indeed, the hCA II/108 complex was stabilized by a number of van der Waals interactions between the inhibitor alkyl moiety and the hydrophobic residues lying on the short helical region, defined by amino acid residues 130-135, and the loop incorporating residues 198-204 (Figure 38A). These interactions were not conserved in the corresponding hCA IX complex, accounting thus for the lower affinity of the inhibitor toward hCA IX than for hCA II. In particular, the van der Waals interactions involving Leu204 were drastically attenuated in the hCA IX adduct not only by the replacement of the Leu bulky residue with the smaller alanine, but also by the concurrent mutation of Val135 into a Leu (Figures 2 and 38C). In fact, Ala204 in hCA IX, similarly to its counterpart Leu204 in hCA II, is located on the re-entrant side of the loop 198-204, forming a sort of "gate" with residue Leu135, located at the end of the helix. This gate is narrower in hCA IX than in hCA II due to the Val/Leu mutation, thus hampering the access to the inhibitor alkyl moiety. The mutations Phe131/Val and Gly132/

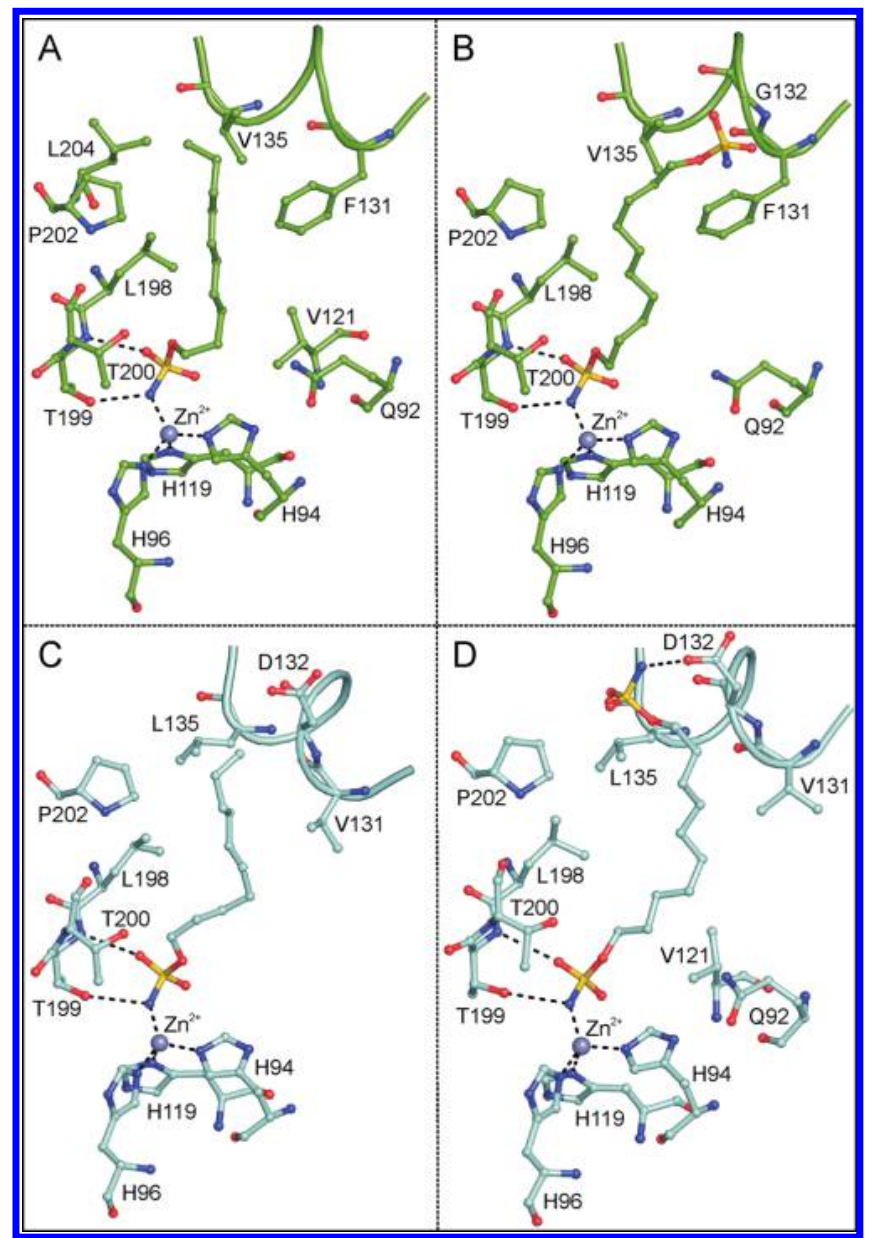

Figure 38. Representation of hCA II active site in complex with (A) 108 (PDB code 3IBU) and (B) 110 (PDB code 3IBN). The panels (C) and (D) show the corresponding adducts of 108 and 110 with hCA IX obtained by a homology modeling study. The ribbon diagram of the helical region $130-135$ is also shown.

Asp also play a key role in lowering the affinity of the inhibitor toward hCA IX with respect to hCA II. In fact, in the first case the shorter side chain of the valine residue weakens the van der Waals interaction with the inhibitor while in the second one, where a neutral residue is substituted by a negatively charged one, a potential destabilizing effect on its interaction with the inhibitor hydrophobic tail was observed. On the contrary, this last mutation determines the higher affinity of compound $\mathbf{1 1 0}$ toward hCA IX. In fact, the second sulfamate group of the inhibitor, which in the adduct with hCA II forms only hydrophobic contacts (Figure 38B), in hCA IX is engaged in a hydrogen bond interaction with the aforementioned Asp132 residue (Figure 38D). This additional hydrogen bond balances the less favorable van der Waals interactions occurring in the hCA IX/110 complex with respect to the hCA II/110 adduct and is responsible for the inversion observed in the affinities of compounds 108 and 110 for the two isoforms. ${ }^{252}$

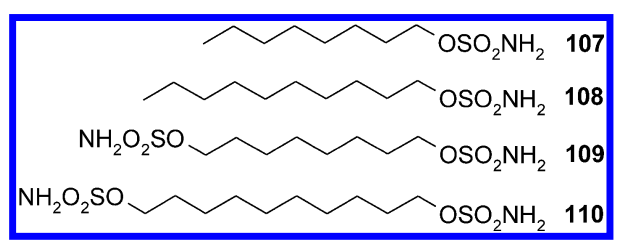


Altogether these findings seem to indicate the region incorporating amino acid residues $130-136$ as a "hot spot" to be considered in structure-based drug design of CA IXselective inhibitors. Indeed, major differences in such residues present in the tumor-associated isoform compared to the cytosolic ones were evidenced, in addition to different interactions in which the tails of the inhibitors participate. ${ }^{252}$

4.5.2. Sulfamate CAls Also Acting as Steroid Sulfatase and Aromatase Inhibitors. Steroid sulfatase (STS) ${ }^{253,254}$ represents a rather new therapeutic target for the treatment of hormone-dependent breast cancer, since it is involved in the production of estrogenic steroid hormones which promote the growth and development of this type of cancer. For this reason several STS inhibitors have been developed in the last years, most of which have an aryl sulfamate ester as their active pharmacophore. ${ }^{253}$ The presence of a sulfamate group in such molecules confers them the capability to interact simultaneously also with CA active site. This has a double effect: on one hand, the interaction with hCA II, which is highly express in red blood cells, is believed to improve the oral bioavailability and pharmacokinetics of such molecules, and to provide a protection against first pass metabolism. ${ }^{255}$ On the other hand, the interaction with hCA IX, ${ }^{249}$ whose expression is increased in many tumors, suggests that the overall anticancer efficacy of this class of drugs could be due to the simultaneous inhibition of this enzyme. Consequently in the last years, many X-ray structures of these compounds in complex with hCA II have been reported, in order to investigate in detail the interaction of sulfamate-based STS inhibitors with CAs. ${ }^{256-263}$

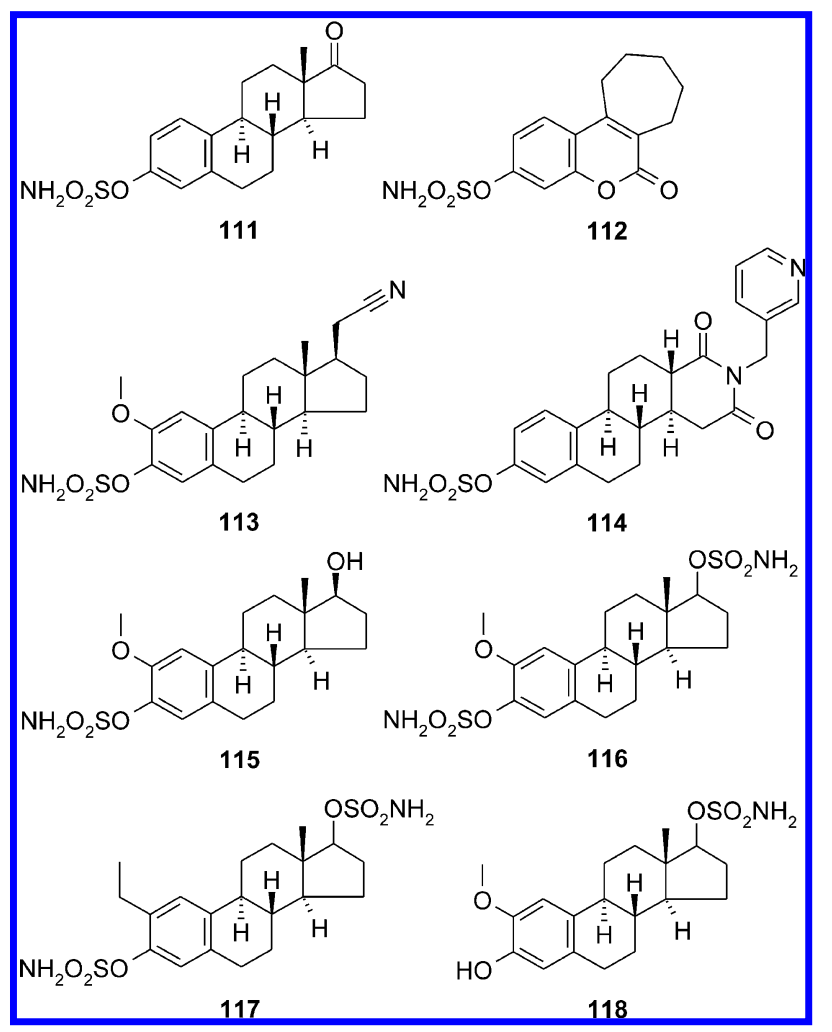

The steroid sulfamates were among the first type of STS inhibitors discovered. Among them, estrone 3-O-sulfamate (EMATE) 111, reported by Potter's group in 1994, is one of the most potent STS inhibitors. ${ }^{264}$ However, this compound was shown to be extremely estrogenic in rodents, and thus not adapted for clinical development. ${ }^{265}$ Several alternative approaches were thus utilized to develop EMATE analogues, devoid of these side effects. Among these approaches it should be mentioned the development of nonsteroidal analogues, which led to the tricyclic 667-coumate 112, an STS inhibitor currently in phase I clinical trials for the treatment of breast cancer, ${ }^{254,257,266-268}$ as well as the modification of the EMATE scaffold and/or its substituent pattern, to give derivatives of type 113-118. ${ }^{257,259-261,263,269,270}$ The X-ray crystal structures of the adducts of all these compounds with hCA II were reported, ${ }^{256,257,259-261,263}$ showing that these molecules bind to hCA II active site coordinating to the $\mathrm{Zn}^{2+}$ ion by means of the deprotonated $\mathrm{NH}^{-}$moiety of the sulfamate group (Figure 39). The steroid ring system of 111 and 113-115 and the tricyclic ring structure of $\mathbf{1 1 2}$ are rather well superposable, when bound to the enzyme active site (Figure 40), with the steroidal backbone of 113 and 115 rotated of $180^{\circ}$ with respect to the others, as a consequence of the presence of the 2-substituent. All these scaffolds lie in the hydrophobic part of the active site, establishing a large number of strong van der Waals interactions (Figure 39A,B,C,D,E). Compound 114 makes an additional hydrogen bond interaction between the nitrogen atom of the pyridine moiety and residue Gln136 (Figure 39D). A completely different orientation was observed for compounds 116-118 (Figure 39F,G,H and Figure 41). Indeed, these molecules bind to the $\mathrm{Zn}^{2+}$ ion with the sulfamate group in position 17 and not that in position 3 as observed for 111 and 113-115. The steroid skeletons of inhibitors 116 and 117 overlap very well, as expected from their structural similarity, while 118 presents small differences in the angles of its ring system compared to the other two derivatives.

The comparison of the inhibition properties of all these compounds and the analysis of their crystal structures allowed to derive several important features on their binding behavior. Thus, compounds having only one sulfamate group on the benzene ring without any substituent in position 2, such as 111, 112, and 114, were the most effective CAIs $\left(\mathrm{IC}_{50}\right.$ in the range $0.1-56 \mathrm{nM}) .^{261,263}$ The presence of a substituent in position 2 reduced considerably the affinity, as observed for $\mathbf{1 1 3}$ and $\mathbf{1 1 5}$ $\left(\mathrm{IC}_{50}=1500 \text { and } 2113 \mathrm{nM} \text {, respectively }\right)^{260,263}$ The introduction of a second sulfamate group in position 17 (116 and 117) led to compounds with a good inhibitory action ( $\mathrm{IC}_{50}$ $=662$ and $169 \mathrm{nM}$, respectively). ${ }^{259,263}$ In these cases, although the ionization of the sulfamate in position 17 is unfavorable with respect to that of the sulfamate in position 3, the 2substituent could make more difficult the interaction of the 3$O$-sulfamate group with the zinc ion, due to a steric hindrance. $^{259}$ Finally, when the inhibitor contains only the sulfamate group in position 17 (118), the efficacy decreases $\left(\mathrm{IC}_{50}=770 \mathrm{nM}\right)$ with respect to the corresponding bissulfamates. ${ }^{263}$ The higher inhibitory activity of bis-sulfamates might be due to the capability of both sulfamate groups to coordinate to the $\mathrm{Zn}^{2+}$ ion, but in the X-ray structures discussed here only one complex was observed. ${ }^{259,263}$

An alternative approach for the treatment of the hormonedependent tumors consists of reducing levels of estrogens using aromatase inhibitors. Such molecules contain as active pharmacophore a heme-chelating azole ring, such as triazole. ${ }^{258}$ Since aromatase inhibitors given in conjunction with STS inhibitors could maximize estrogen depletion in the treated patients, new hybrid drugs able to inhibit simultaneously both enzymes have been designed, originating in dual aromatasesteroid sulfatase inhibitors (DASIs). The first examples of DASIs are represented by compounds such as 4-[(4-O- 


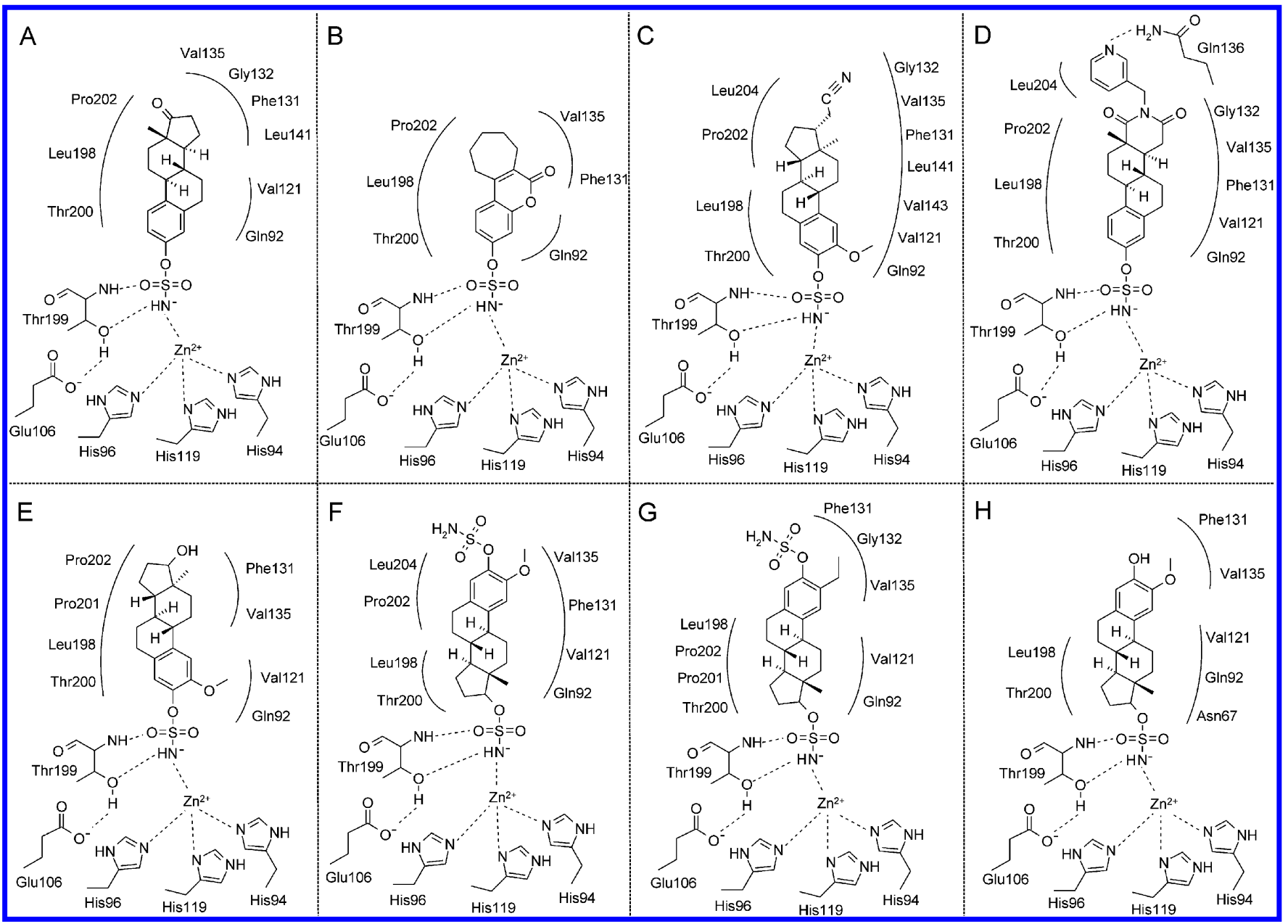

Figure 39. Schematic view of hCA II active site in complex with: (A) 111 (PDB file was kindly provided by Claudiu Supuran), (B) 112 (PDB code 1TTM), (C) 113 (PDB code 3BET), (D) 114 (PDB code 3C7P), (E) 115 (PDB code 2X7U), (F) 116 (PDB code 2GD8), (G) 117 (PDB code 2X7T), and (H) 118 (PDB code $2 X 7 S$ ).

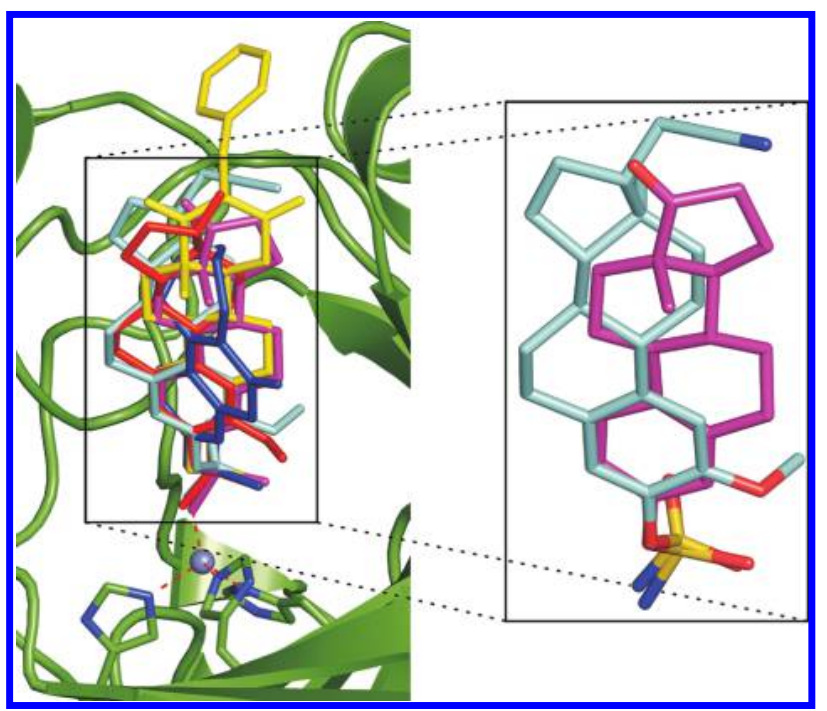

Figure 40. Superposition of hCA II/inhibitor adducts: 111 is reported in magenta, 112 in blue, 113 in cyan, 114 in yellow, and 115 in red. On the right side the enlarged view of 111 and 113 inhibitors within hCA II active site shows the different orientation of their backbone.

sulfamoylbenzyl)(4-cyanophenyl)amino]-4H-[1,2,4] triazole 119 and 4-[(3-bromo-4-O-sulfamoylbenzyl)-(4-cyanophenyl)-

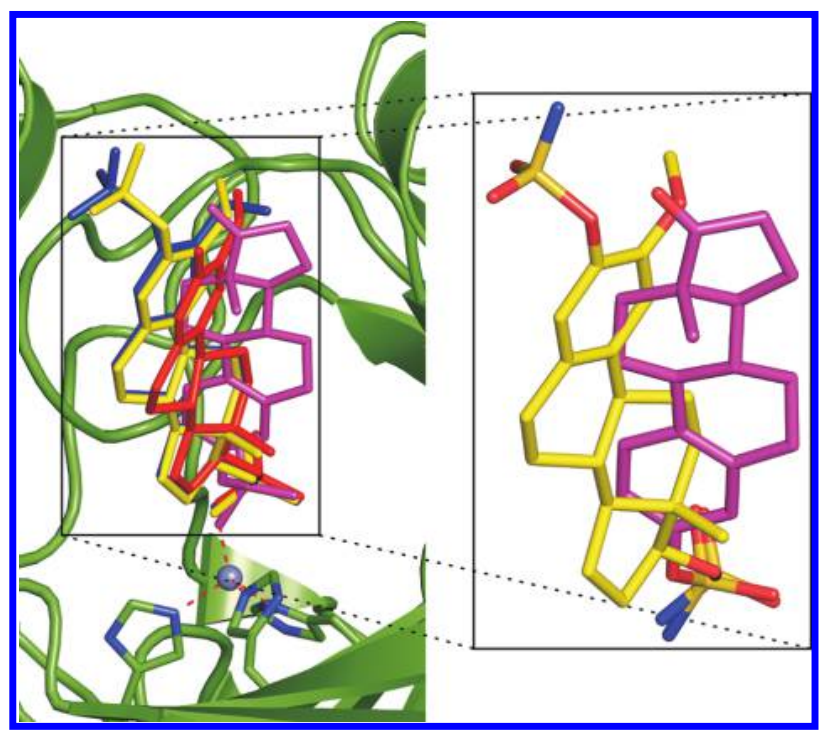

Figure 41. Structural superposition of hCA II/inhibitor complexes: 111 is shown in magenta, 116 in yellow, 117 in blue, and 118 red. On the right side the zoom of $\mathbf{1 1 1}$ and $\mathbf{1 1 6}$ inhibitors within hCA II active site shows the different orientation of their backbone.

amino]-4H-[1,2,4]triazole $120 .^{271}$ As already observed for the aforementioned STS inhibitors, these molecules, containing 
<smiles>N#Cc1ccc(N(Cc2ccc(OS(=O)(=O)O)cc2)n2cnnc2)cc1</smiles>

also a sulfamate functionality, are able to bind the CA II active site. In particular, 119 showed an $\mathrm{IC}_{50}$ value of $27 \mathrm{nM}$ against CA II, while 120 was $4-5$ times less potent, with an $\mathrm{IC}_{50}$ value of $137 \mathrm{nM}^{258}$ A detailed description of the molecular interactions between these DASIs and CA II was provided by resolving the crystal structures of the corresponding complexes. $^{258}$ In particular, both molecules were located within the active site cavity coordinating the zinc catalytic ion and stabilized by van der Waals interactions with several protein residues. The binding of these compounds to the enzyme was fairly similar, but not identical. In particular, the introduction of the bromine in compound $\mathbf{1 2 0}$ caused the rotation of the sulfamate-bearing aromatic ring by about $30^{\circ}$, due to a steric hindrance between the protein and the bulky bromine atom (Figure 42). Thus, the positioning of the sulfamate containing

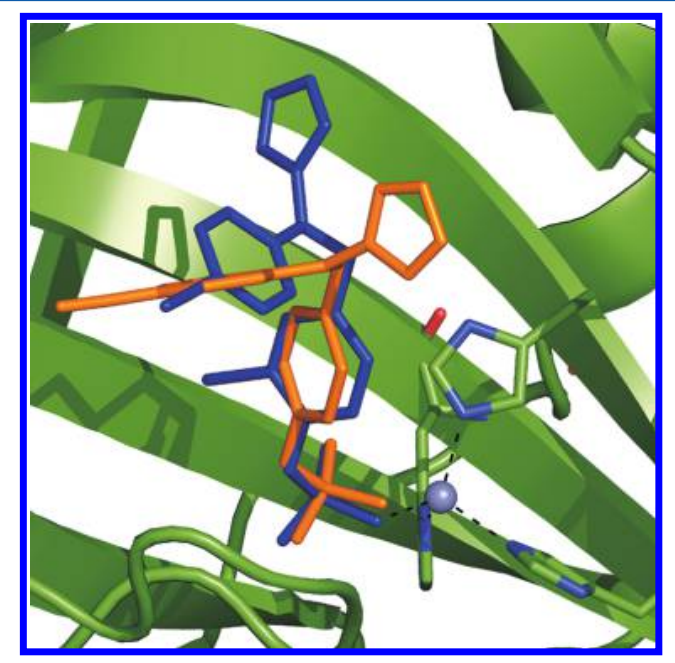

Figure 42. Structural superposition of 119 (orange) (PDB code $1 \mathrm{XPZ}$ ) and 120 (blue) (PDB code $1 \mathrm{XQO}$ ) when bound to the hCA II active site.

aromatic ring and accommodation of the large bromine atom appeared to be the major factors that resulted in a significantly lower affinity of $\mathbf{1 2 0}$ toward the CA II. More recently a new class of DASIs, based on a biphenyl template, has been reported, with one of the most potent compound being 3chloro-2'-cyano-5'-(1H-1,2,4-triazol-1-ylmethyl)biphenyl-4-ylsulfamate 121. ${ }^{262,263}$ As already observed for 119 and 120, this compound, bearing a sulfamate group, is able to bind the hCA II $\left(\mathrm{IC}_{50}\right.$ value of $86 \mathrm{nM}$ ). The X-ray crystal structure of the hCAII/121 adduct was solved, showing the biphenyl moiety located at the center of active site cavity and the triazole ring pointing toward a small cavity positioned close to the entrance of the active site, and delimited by Leu60, Asn62, and Glu69 ${ }^{262}$ (Figure 43).

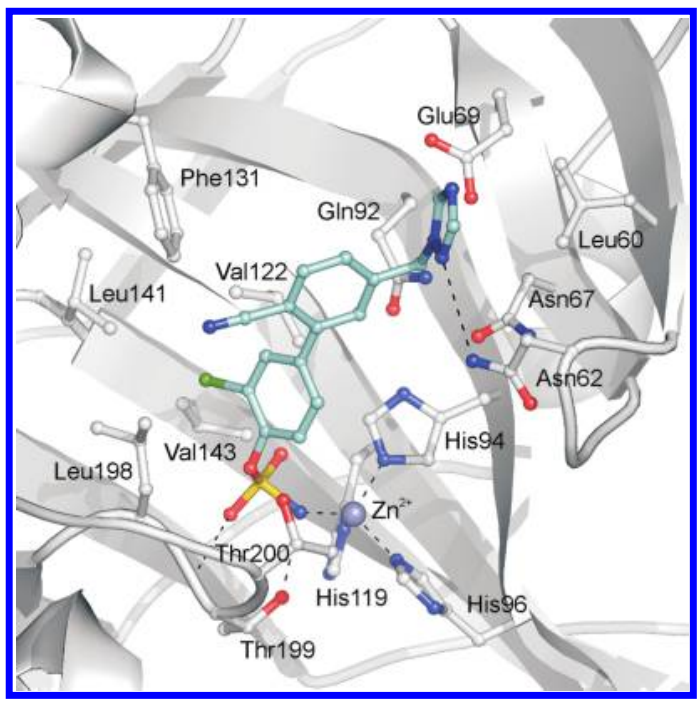

Figure 43. Active site view of hCA II/121 adduct (PDB code 2WD3). The enzyme is represented as ribbon diagram in gray.

In summary, the analysis of the structures of the hCA IIsulfamates complexes confirmed that even small modifications to the inhibitor structures can induce large modifications in the binding mode and affinity for the various CA isoforms. Thus, the knowledge of the interactions between sulfamate-based ligands and hCA II could allow to support more rational drug design strategies.

\subsection{Sulfonamides/Sulfamates/Sulfamides Containing Sugar Moieties}

Among the different strategies so far developed for designing selective CAIs, the "sugar approach" represents one of the most interesting and versatile tools. This approach, which has led to the development of numerous potent inhibitors of potential clinical use, ${ }^{272-274}$ consists in the incorporation of glycosyl moieties into different CAI scaffolds. CAIs obtained with this approach can be schematically grouped in two major classes: the first class contains compounds where the carbohydrate moiety is directly attached to the sulfonamide/sulfamide/ sulfamate group (see compounds 122-127), while the second class is characterized by molecules where the carbohydrate moiety is attached to the organic scaffold of aromatic sulfonamides (see compounds 128-130). ${ }^{273,275-278}$

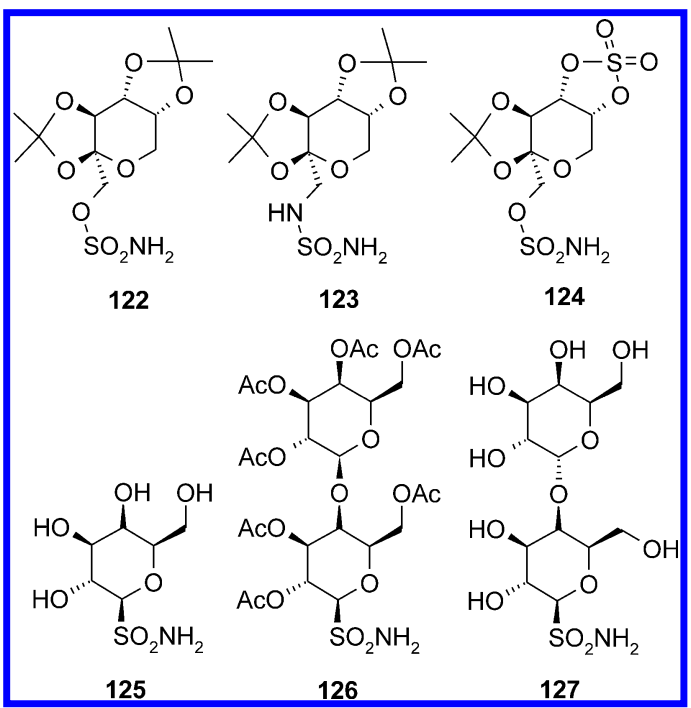


The marketed antiepileptic drug topiramate 122 and its analogues 123 and 124 are interesting examples of compounds belonging to the first class. Topiramate, 2,3,4,5-di-O-isopropylidene- $\beta$-D-fructopyranose sulfamate, presents a very peculiar chemical structure, consisting of a monosaccharide moiety bearing a sulfamate functional group. This drug possesses potent anticonvulsant effects, which can be ascribed to a multifactorial mechanism of action: blockade of sodium channels and kainate/AMPA receptors, $\mathrm{CO}_{2}$ retention secondary to inhibition of the red cell and brain CAs, as well as enhancement of GABA-ergic transmission. ${ }^{279-284}$ It was also recently demonstrated that topiramate is able to induce weight loss in obese epilepsy patients after pharmacological treatment. ${ }^{285}$ Since the use of other CAIs was also associated with weight loss, ${ }^{204}$ it was supposed that the antiobesity properties of $\mathbf{1 2 2}$ could be due to the inhibition of the CAs involved in the biosynthesis of lipids. According to this hypothesis, topiramate was demonstrated to act as very potent inhibitor of several CA isozymes, among which hCA II $\left(K_{\mathrm{I}}=13.8 \mathrm{nM}\right)$ and hCA VA $\left(K_{\mathrm{I}}=25.4 \mathrm{nM}\right){ }^{286,287}$ The structural determinants responsible of the high affinity of $\mathbf{1 2 2}$ for these CAs were investigated by means of a combined structural and theoretical approach. ${ }^{286,287}$ In particular, the X-ray crystal structure of the hCA II/122 complex was solved ${ }^{286}$ and used as template for a homology modeling study on the adduct that $\mathbf{1 2 2}$ forms with hCA VA. ${ }^{287}$ The main protein-inhibitor interactions observed in these complexes are schematically depicted in Figure 44. These studies highlighted that whereas the sulfamate group of the inhibitor binds to the active site of both enzymes in a similar manner, a different network of polar interactions of the sugar scaffold distinguishes the hCA VA/122 adduct from the hCA II/122 one. These differences can justify the diverse binding affinities of 122 toward the two CA isozymes, ${ }^{287}$ and offer interesting perspectives for the design of antiobesity CAIs using topiramate as lead compound.

Compound $123,{ }^{288}$ the sulfamide analogue of 122 from which differs only for the substitution of an oxygen atom with a $\mathrm{NH}$ moiety, is a much less potent hCA II inhibitor. The analysis of its crystal structure in complex with hCA II correlates well with this finding ${ }^{288}$ Indeed, although 123 binds to the CA II active site in a conformation very similar to that of the sulfamate analogue, one of its methyl groups makes a clash with the methyl side chain of Ala65, ${ }^{288}$ thus accounting for the 2 orders of magnitude lower affinity toward the enzyme. Compound $124^{289}$ is another structural analogue of topiramate, where one of the di-isopropylidene moieties was substituted by a cyclic sulfate group. The X-ray structure of the hCA II/124 complex has also been reported, ${ }^{290}$ showing that this inhibitor binds to the enzyme active site in a completely different way as compared to $\mathbf{1 2 2}$ and 123. In particular, while the sulfamate/ sulfamide groups of these three inhibitors are well superposable, the sugar moiety of 124 is rotated by about $180^{\circ}$ with respect to those of $\mathbf{1 2 2}$ and $\mathbf{1 2 3}$ (Figure 45). This rotation is responsible for a different pattern of hydrogen bond interactions within the enzyme active site and consequently for a diverse inhibition profile of the three compounds toward hCA II (and other CA isoforms). ${ }^{288,289}$ Altogether these findings highlighted again that even minor structural differences in these sugar moieties can be responsible for very different binding modes to the $\mathrm{CA}$ active site and consequently for very different inhibitory effects.

Recently, several other examples of sugar derivatives belonging to the same class as 122-124 have been reported ${ }^{291}$

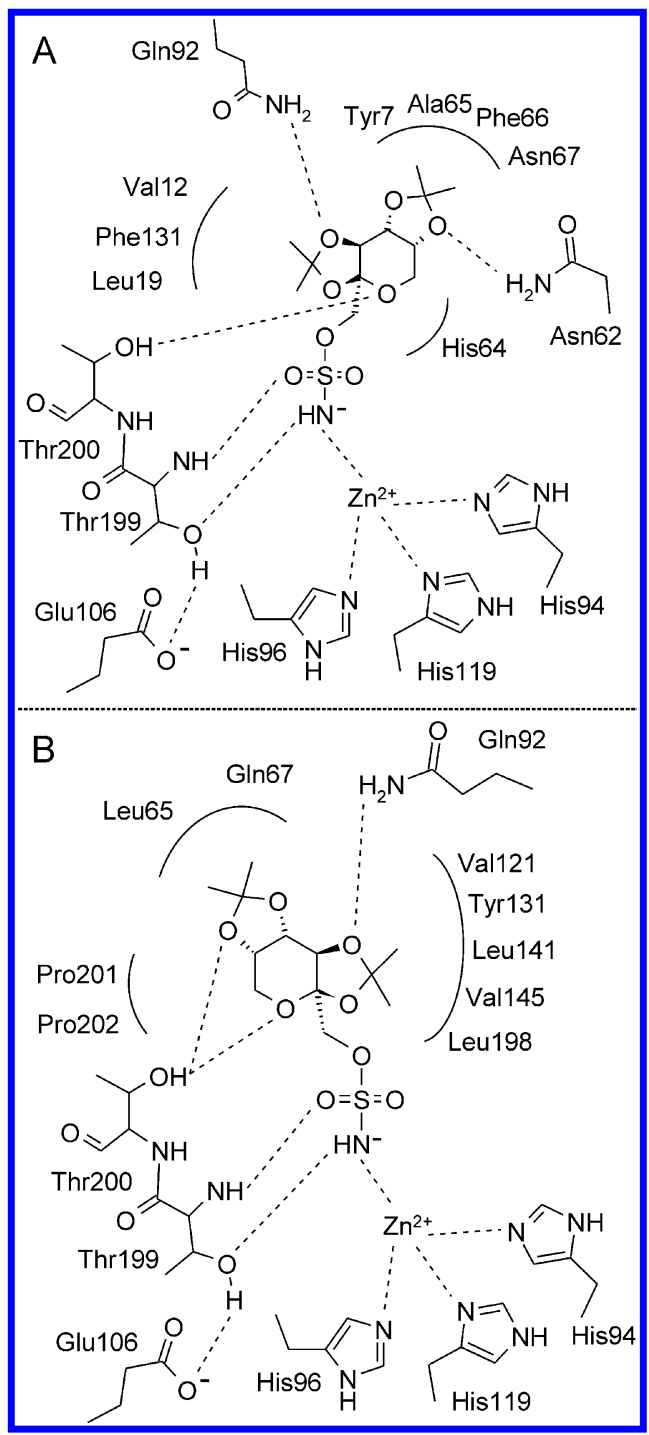

Figure 44. Schematic active site representation of (A) hCA II/122 crystal structure (PDB code 3HKU) and (B) hCA VA/122 adduct obtained by a homology modeling study.

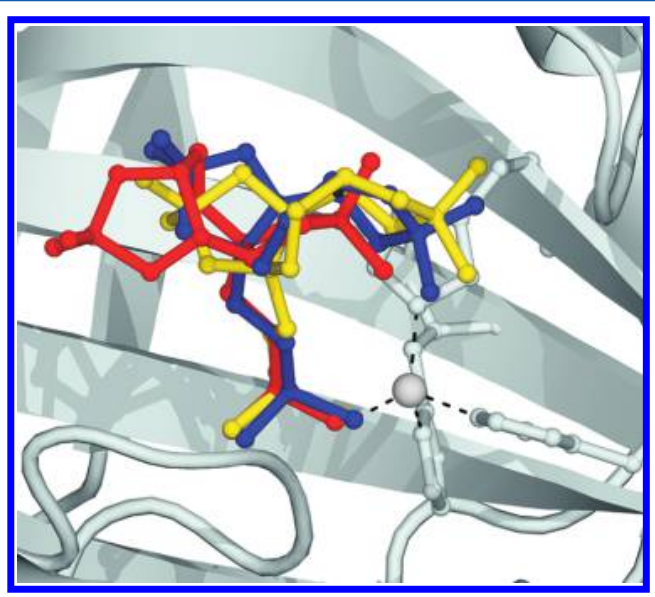

Figure 45. Structural superposition between hCA II/122 (yellow, PDB code $3 \mathrm{HKU}$ ), hCA II/123 (blue, PDB code 2H15), and hCA II/ 124 (red, PDB code 1EOU) adducts.

(see as examples compounds 125-127). These molecules, synthesized starting from per-O-acetylated sugar moieties, ${ }^{292}$ 


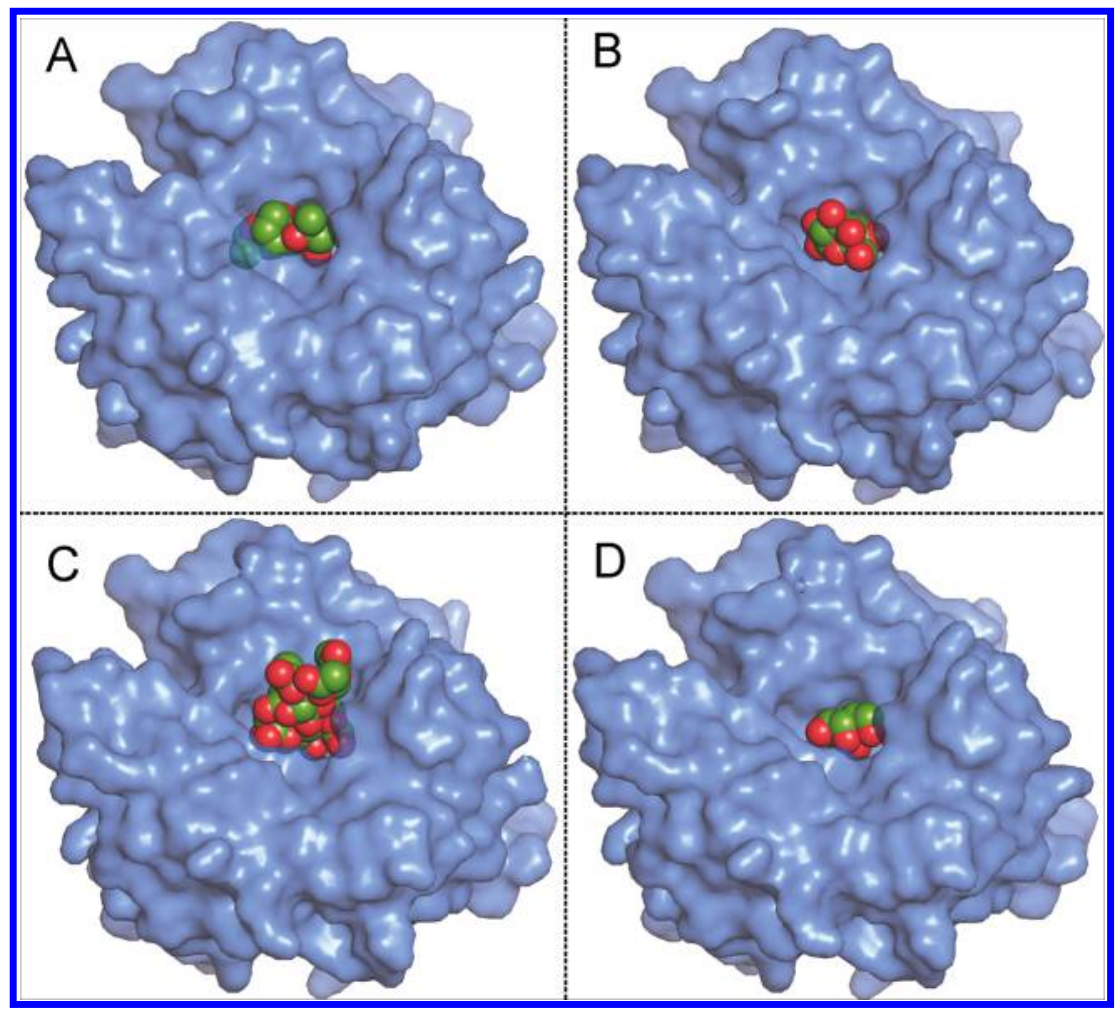

Figure 46. View of the shape complementarities between hCA II active site and (A) 122 (PDB code 3HKU), (B) 125 (PDB code $3 \mathrm{HKQ}$ ), (C) 126 (PDB code $3 \mathrm{HKN}$ ), and (D) 127 (PDB code $3 \mathrm{HKT}$ ). The inhibitor atoms are shown as spheres and the enzyme is represented as a surface-model.

contain a primary sulfonamide group directly attached to the anomeric center of a mono- or disaccharide fragment. In terms of chemical structure, these compounds represent an unrelated CAI class, since to date, no other inhibitors containing a methine sulfonamide moiety $\left(\mathrm{RR}^{\prime} \mathrm{CH}-\mathrm{SO}_{2} \mathrm{NH}_{2}\right)$ have been reported. Moreover, the observation that these compounds presented very poor passive membrane permeability ${ }^{291}$ made them interesting alternatives to the classical aromatic CAIs, also offering attractive perspectives for the design of inhibitors able to target only extracellular CA isoforms. Unfortunately, kinetic studies on these molecules did not fulfill the expectations, demonstrating both that they were only moderate CAIs, with $\mathrm{K}_{\mathrm{I}} \mathrm{s}$ in the micromolar range, and that they possessed a very flat inhibition profile across all CA isozymes investigated. ${ }^{291}$ This result was quite surprising especially compared to what observed both for the classical CAIs and for the structural related analogue topiramate, which instead presented $K_{\mathrm{I}}$ values in the low nanomolar range for several CA isoforms. Thus, a structure-based approach was used to investigate the structural features of these compounds responsible for their weak CA inhibitory properties. In particular, high resolution X-ray crystal structures were obtained for hCA II in complex with one monosaccharide $\mathbf{1 2 5}$ and two disaccharide derivatives (the per$O$-acetylated sugar 126 and the fully deprotected sugar 127) and compared with the X-ray structure of the hCA II/122 complex. This comparison explained well the above-reported differences in the kinetic behavior of such compounds. Indeed, despite the similarity in chemical structure with 122, none of the three anomeric sulfonamides was able to form as many direct interactions with protein residues as topiramate. ${ }^{291}$ This finding is clearly related to the inhibitor overall shapes. Indeed topiramate shows a peculiar " $T$ " shape, with the sugar scaffold spanning the diameter of the active site about $5 \AA$ above the catalytic zinc ion. On the contrary, the anomeric sulfonamides present an elongated shape, resulting in less bulky inhibitors which are not able to efficiently interact with active site residues (Figure 46). ${ }^{291}$ These data suggest that further chemical modifications have to be introduced into the carbohydrate skeleton of the anomeric sulfonamides, to optimize the protein/inhibitor binding and enhance the enzyme inhibition activity.

The derivatization of 4-carboxy-benzenesulfonamide 12 or sulfanilamide $\mathbf{5}$ with tails incorporating different sugar moieties led to compounds of type 128-130 belonging to the second class of sugar containing CAIs (see above). These compounds were developed within a general research project aimed at developing CAIs with applications as antiglaucoma agents. In agreement with the design hypothesis, these molecules showed very good CA II inhibitory activity, ${ }^{272-274,276}$ and possessed interesting physicochemical properties, such as water solubility, good penetrability through the cornea, etc., that made them good candidates as topically acting antiglaucoma drugs to be administered directly into the eye. ${ }^{275}$

One of the most promising compound of this series was $\mathrm{N}$ (4-sulfamoylphenyl)- $\alpha$-D-glucopyranosylamine (glucose derivative 130). Indeed, this molecule was shown to be a potent inhibitor of several CA isozymes and a promising antiglaucoma agent with topical activity in an animal model of the disease. ${ }^{276}$ The crystallographic structure of $\mathbf{1 3 0}$ in complex with hCA II was also solved providing insights into the molecular basis of its strong enzyme inhibitory activity. ${ }^{278}$ In particular, while the sulfonamide and the phenyl ring of the inhibitor bound in the canonical way to the enzyme, the glycosyl moiety, responsible for the high water solubility of the compound, was oriented toward the hydrophilic region of the active site, stabilized by a network of hydrogen bonds with five water molecules and the 


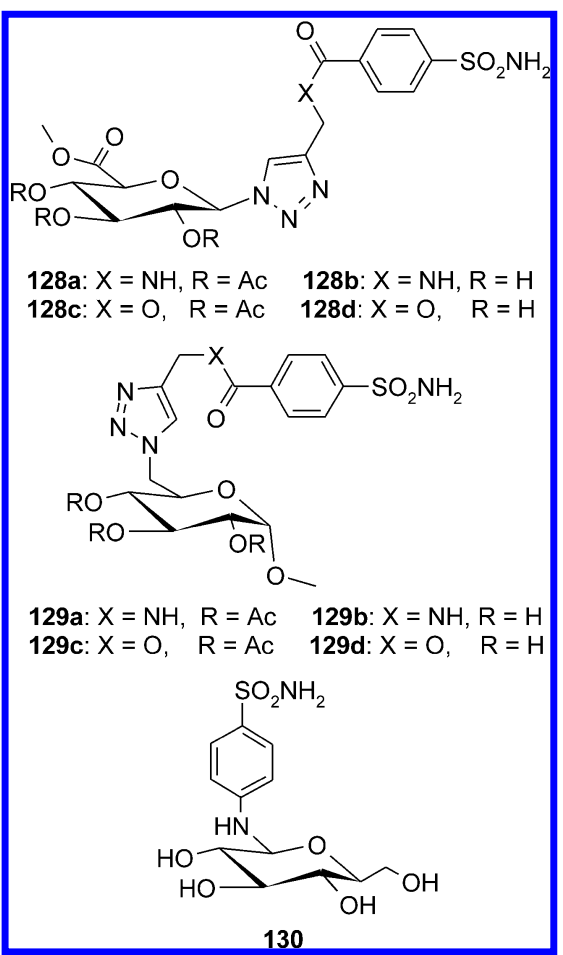

enzyme residues Gln92 and Pro201 (Figure 47). ${ }^{278}$ It is interesting to observe that the type of interactions observed in

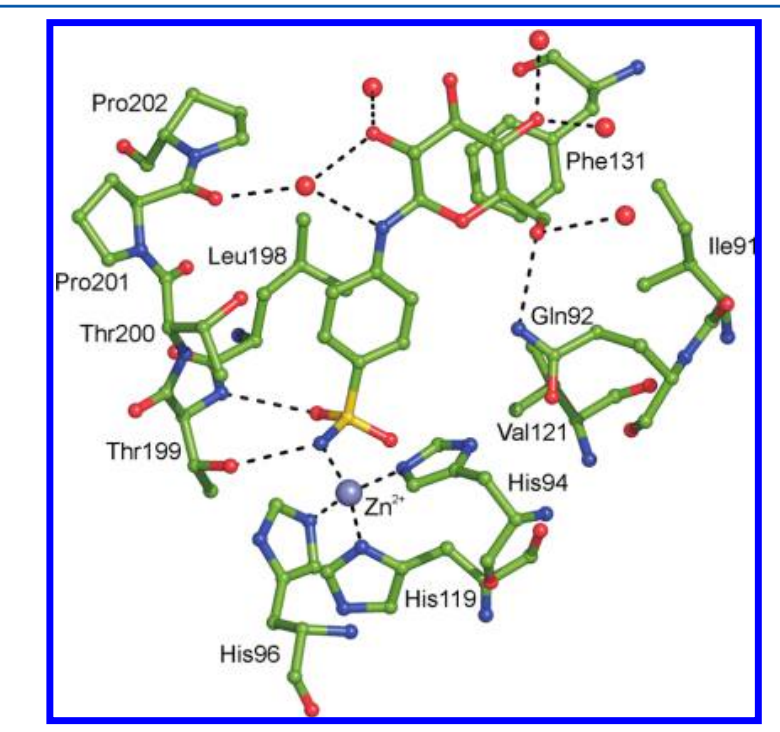

Figure 47. Representation of the active site in the hCA II/130 complex (PDB code 2HL4) showing residues participating in recognition of the inhibitor molecule. Hydrogen bonds and the active site $\mathrm{Zn}^{2+}$ coordination are also shown. Water molecules are shown as red circles.

the hCAII/130 adduct was completely different from those reported for the other sugar derivatives reported above and thus this could be suitably used for the rational drug design of other sugar-based CAIs.

\section{INSIGHTS INTO CA INHIBITION: STRUCTURAL FEATURES OF NON-ZINC-BINDING INHIBITORS}

An increasing number of different chemotypes than the sulfonamides and their isosteres has been recently reported to act as CAIs. Among them are the phenols ${ }^{293-295}$ (some of which are natural compounds), ${ }^{296}$ the polyamines, ${ }^{297}$ the coumarins, $^{298,299}$ and the antiepileptic drug lacosamide. ${ }^{300}$ Interestingly, all these type of derivatives, differently from sulfonamides and their isosteres, bind to the CA active site not interacting directly with the catalytic zinc ion. In particular, phenols ${ }^{295,296}$ and polyamines ${ }^{297}$ are anchored to the zinccoordinated water molecule/hydroxide ion, whereas the recently reported class of effective and in some cases isoformselective CAIs, the coumarins and thiocoumarins, ${ }^{298,299}$ binds (in hydrolyzed form) far away from the zinc ion, not interacting at all with it, partially occluding the entrance to the active site. The antiepileptic lacosamide ${ }^{300}$ binds similarly to the hydrolyzed coumarins, although it bears no chemical similarity at all with them. The main structural features of all these classes of inhibitors will be summarized in the next paragraphs.

\subsection{Compounds Anchoring to the Zinc Bound Water Molecule}

5.1.1. Phenols. Phenols constitute a class of compounds which over the years have had different applications as pharmacological agents in the treatment of a wide variety of diseases. Indeed, they have been reported to possess anticancer, antioxidant, antimutagenic, antibacterial, antiviral or antiinflammatory activities. ${ }^{301-312}$

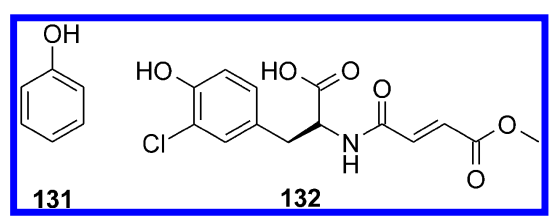

The simplest member of this family of compounds is phenol 131, which as far as in 1982 was discovered to be a competitive inhibitor of human CA II by Lindskog's group. ${ }^{293,294}$ Subsequently, the X-ray crystal structure of its adduct with hCA II was solved, providing structural reasons of such a kinetic behavior. ${ }^{295}$ In particular, the analysis of the structure revealed the binding of two 131 molecules to the enzyme. The first one was located in a hydrophobic patch about $15 \AA$ away from the catalytic zinc ion and close to a symmetry-related molecule. ${ }^{295}$ However, since this location does not correspond either to the molecular trajectory of $\mathrm{CO}_{2}$ or secondary $\mathrm{CO}_{2}$ binding sites predicted in molecular dynamics calculation$\mathrm{s},{ }^{79,313}$ it is likely that this binding does not interfere with CA II catalytic activity. A second phenol molecule was found within the active site, showing a completely new binding mode. Indeed, this molecule did not coordinate to the zinc ion, but was anchored to the active site through two hydrogen bonds of the $\mathrm{OH}$ moiety with the zinc-bound water/hydroxide ion and the NH amide of Thr199 (Figure 48). Furthermore, the aromatic ring was found to lie in a hydrophobic pocket of the active site, delimited by residues Val121, Val143, Leu198, and Trp209 (Figure 48). These findings, together with the observation that 131 was a competitive CA II inhibitor, provided the first evidence, very recently confirmed by two independent structural studies, ${ }^{69,70}$ that the hydrophobic pocket delimited by Val121, Val143, Leu198, and Trp209 constitutes the $\mathrm{CO}_{2}$ binding pocket. It is worth noting that this crystal structure represented the first direct evidence that phenol binds to the enzyme without altering the coordination of the zinc ion, confirming the results obtained indirectly by previous spectroscopic studies of $\mathrm{Co}$ (II)-substituted CA II and its phenol complex, ${ }^{293}$ and by molecular modeling studies based on ${ }^{13} \mathrm{C}$ NMR data. ${ }^{314}$ 


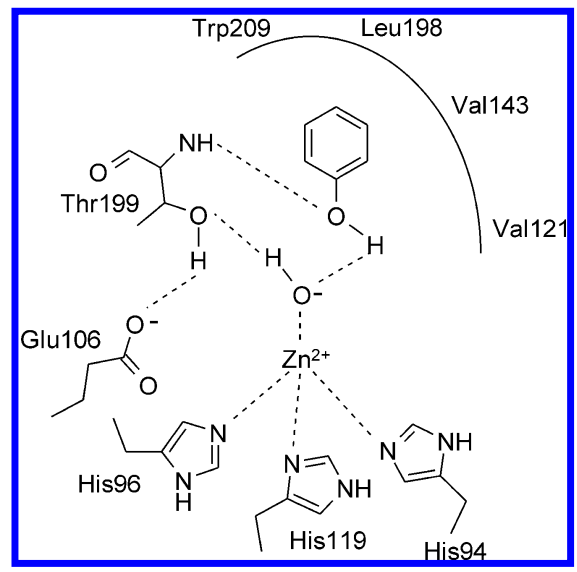

Figure 48. Schematic representation of the binding mode of 131 to the hCA II active site. $\mathrm{Zn}^{2+}$ coordination, polar and hydrophobic interactions (distance $<4.5 \AA$ ) are also reported.

More recently, additional structural information on the binding mode of phenolic derivatives to the CA active site has been obtained. In particular, xylariamide A 132, a phenolic natural product, which acts as micromolar hCA II inhibitor $\left(K_{\mathrm{I}}\right.$ $=8 \mu \mathrm{M})$ and possesses a methyl ester moiety, has been cocrystallized with hCA II. $^{296}$ The main hCA II/132 interactions, as derived from the analysis of this structure, are shown in Figure 49. Surprisingly, differently from 131, compound $\mathbf{1 3 2}$ is anchored to the zinc bound water molecule, not through the $\mathrm{OH}$ phenolic moiety but by means of a hydrogen bond involving the carbonyl oxygen of the ester functionality, which also makes a second hydrogen bond with the Thr199NH atom. The methyl moiety of the ester

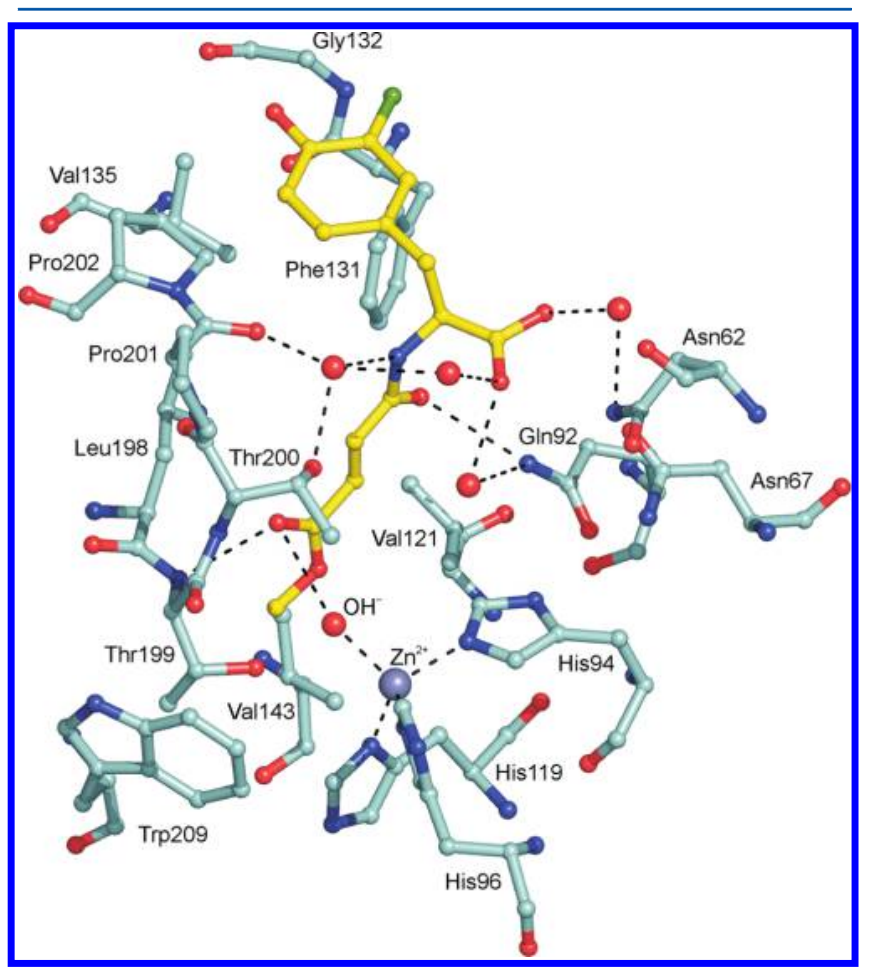

Figure 49. Active site view of hCA II/132 adduct (PDB code 3P4V) showing residues participating in recognition of the inhibitor molecule. Only one conformation of the chlorophenol moiety is reported. Water molecules are shown as red circles. functionality is in van der Waals contact with Trp209. Various oxygen/nitrogen atoms of the inhibitor make additional direct or solvent-mediated hydrogen bonds with several enzyme residues, such as Thr200, Gln92, Pro201, and Asn62, whereas the chlorophenol moiety was slightly disordered (two conformations were observed), making only van der Waals contacts with CA II active site. These data, together with several recently reported inhibition studies against all $\alpha$-CA isozymes on synthetic or natural phenolic products, ${ }^{315-319}$ strongly suggest that phenol 131 represents a lead compound that can be utilized to obtain novel CAIs, different from classical sulfonamides and their isosteres. In particular, different substitution patterns of such lead can be used to significantly modulate the binding mode and consequently affinity and selectivity toward different CAs.

5.1.2. Spermine and Related Polyamines. Several kinetic and X-ray crystallographic studies demonstrated that amines such as histamine or L-adrenaline were able to effectively activate many CA isoforms. ${ }^{320-331}$ Indeed, these molecules were shown to bind at the entrance of the CA active site facilitating, through their protonatable groups, the rate limiting step of the catalytic cycle: the deprotonation of the $\mathrm{Zn}^{2+}$ bound water molecule with the formation of the zinc hydroxide species of the enzyme (see Introduction). ${ }^{70,72-75} \mathrm{On}$ the basis of these observations it was suggested that polyamines, such as spermine 133 , spermidine $134, N, N^{\prime}$-bis(3-aminoethyl)-1,3-propanediamine 135 and triethylenetetramine 136, could also act as CA activators, since they possessed 3-4 primary/secondary amine groups in their molecules, with $\mathrm{p} K_{\mathrm{a}} \mathrm{s}$ in the range 7.9-10.9. Surprisingly, recently reported kinetic studies demonstrated that these molecules did not have at all activating properties against hCA isoforms, but on the contrary showed significant inhibitory features. ${ }^{297}$ Moreover, each of these polyamines showed a diverse inhibition profile against different CA isozymes, probably reflecting several factors controlling activity, such as the length of the aliphatic chain of the polyamine, the number of amine functionalities present in it, and presumably the charged character (as ammonium cations) of these molecules. Indeed, the different 12 catalytically active isoforms were inhibited with potencies ranging from the low nanomolar to the millimolar range by 133 and 134, whereas the synthetic compounds 135 and 136 showed weaker inhibition, with affinities in the micromillimolar range (Table 5).

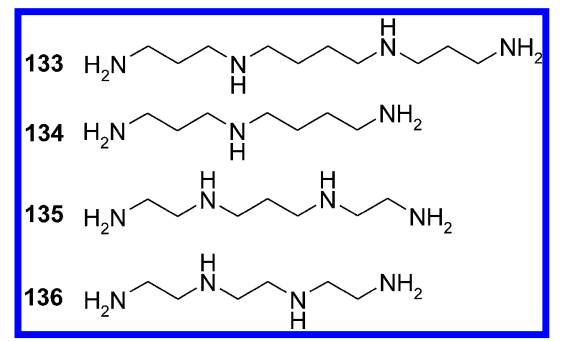

To better understand the inhibitory mechanism of this new class of CAIs, the X-ray crystal structure of spermine 133 in adduct with hCA II was solved. ${ }^{297}$ The main protein-inhibitor interactions observed in this structure are schematically depicted in Figure 50. According to this figure, the polyamine 133, which in the experimental conditions used is probably a tetracation, adopts a coiled conformation when bound to the enzyme active site. It was found bound deep within the hCA II active site, but interestingly it was not directly coordinated to 
Table 5. Inhibition Data of Polyamines 133-136 against CA Isozymes I-XIV by a Stopped-Flow $\mathrm{CO}_{2}$ Hydration Assay Method $^{a}$

\begin{tabular}{ccccc} 
& \multicolumn{4}{c}{$\mathrm{K}_{\mathrm{I}}(\mu \mathrm{M})$} \\
\cline { 2 - 5 } isozyme $^{b}$ & $\mathbf{1 3 3}$ & $\mathbf{1 3 4}$ & $\mathbf{1 3 5}$ & $\mathbf{1 3 6}$ \\
$\mathrm{hCA} \mathrm{I}^{c}$ & 231 & 1.40 & 115 & 100 \\
$\mathrm{hCA} \mathrm{II}^{c}$ & 84 & 1.11 & 75 & 64 \\
$\mathrm{hCA} \mathrm{III}^{c}$ & 167 & 11.5 & 63 & 48 \\
$\mathrm{hCA} \mathrm{IV}^{c}$ & 0.010 & 0.112 & 44 & 35 \\
$\mathrm{hCA} \mathrm{VA}^{c}$ & 0.84 & 1.22 & 50 & 38 \\
$\mathrm{hCA} \mathrm{VB}^{c}$ & 0.83 & 1.44 & 59 & 49 \\
$\mathrm{hCA} \mathrm{VI}^{c}$ & 0.99 & 1.41 & 53 & 43 \\
$\mathrm{hCA} \mathrm{VII}^{c}$ & 0.71 & 1.23 & 58 & 45 \\
$\mathrm{hCA} \mathrm{IX}^{d}$ & 13.3 & 1.37 & 48 & 39 \\
$\mathrm{hCA} \mathrm{XII}^{d}$ & 27.6 & 44.1 & 68 & 57 \\
mCA XIII & 22.6 & 11.6 & 66 & 52 \\
$\mathrm{hCA} \mathrm{XIV}^{c}$ & 0.86 & 1.00 & 36 & 12.1
\end{tabular}

${ }^{a}$ Data were obtained by ref $297 .{ }^{b} \mathrm{~h}=$ human, $\mathrm{m}=$ murine isozyme. ${ }^{c}$ Full length enzyme. ${ }^{d}$ Catalytic domain.

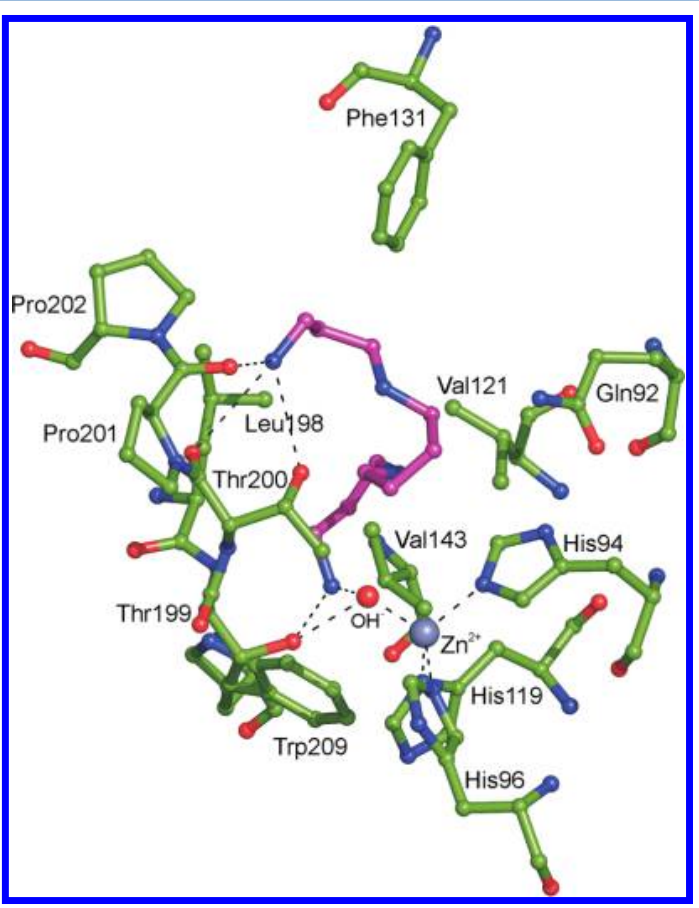

Figure 50. Representation of the active site of the hCA II/133 complex (PDB code 3KWA) showing residues participating in recognition of the inhibitor molecule.

the metal ion. In particular, the polyamine was anchored to the $\mathrm{Zn}^{2+}$ bound hydroxide ion by means of a strong hydrogen bond involving one of its terminal ammonium groups, in a way reminiscent of the binding of phenol 131. ${ }^{295}$ The same moiety participates in a second hydrogen bond with the Thr199OG1 atom. Additional hydrogen bonds with residues Thr200 and Pro201 and several van der Waals contacts with enzyme residues Gln92, Val121, Phe131, Val143, Leu198, Thr199, Thr200, Pro201, and Pro202 further stabilize the adduct (Figure 50). The analysis of this structure clearly shows that spermine binds within the CA active site in a completely different way than classical sulfonamides, justifying the peculiar inhibition profile of this class of new CAIs. These findings open the way to the development of a new class of CAIs with a diverse inhibition mechanism, and hopefully a diverse selectivity profile for various CA isoforms (for example, spermine is a CA IV-selective inhibitor, with binding costants to this isoform in the low nanomolar range, whereas for other ones it binds in the micro- or millimolar range) (Table 5). ${ }^{297}$

5.2. Compounds Located at the Entrance of the Active Site: Coumarins and Lacosamide

In the continuous search of novel chemotypes which could act as efficient CAIs, $\mathrm{Vu}$ and co-workers ${ }^{332}$ recently reported an elegant study where ESI-FTICR-MS techniques were used for the screening of a large number of natural product extracts against bCA II. To the general surprise, this approach led to the identification of a natural product, namely 6-(1S-hydroxy-3methylbutyl)-7-methoxy- $2 H$-chromen-2-one 137, as CA II inhibitor. This result was of a great interest since compound 137 is a coumarin with a chemical structure completely different from all other CAIs investigated earlier, thus constituting a putative valuable lead for the design of new CAIs. Starting from these observations it was evidently necessary to investigate coumarins as a new class of CAIs. For this aim, Supuran's group recently reported a detailed inhibition study of compound 137, and many structurally related analogues, among which 138 and 139, against all catalytically active CAs, ${ }^{298}$ providing a series of very interesting observations. In particular, these studies showed that when an incubation time of 10-15 min was allowed for the formation of the enzyme-inhibitor adduct, as generally done for other types of CAIs, only a weak micromolar to millimolar inhibition was observed; on the contrary, more effective inhibitory properties were observed using higher incubation times $(6 \mathrm{~h})$ (see Table 6). ${ }^{298}$ Indeed, in these conditions coumarins 137-139 were

Table 6. Inhibition Data of Mammalian Isozymes CA I-XIV with Coumarins $137-139$ by a Stopped-Flow $\mathrm{CO}_{2}$ Hydration Assay Method ${ }^{a}$

\begin{tabular}{cccc} 
& \multicolumn{3}{c}{$K_{\mathrm{I}}(\mu \mathrm{M})$} \\
\cline { 2 - 4 } isozyme $^{b}$ & $\mathbf{1 3 7 ^ { e }}$ & $\mathbf{1 3 8 ^ { e }}$ & $\mathbf{1 3 9 ^ { e }}$ \\
hCA I$^{c}$ & 0.08 & 3.1 & 5.9 \\
hCA II $^{c}$ & 0.06 & 9.2 & 0.07 \\
hCA III $^{c}$ & $>1000$ & $>1000$ & 161 \\
hCA IV $^{c}$ & 3.8 & 62.3 & 7.8 \\
hCA VA $^{c}$ & 96.0 & $>1000$ & 645 \\
hCA VB $^{c}$ & 17.7 & 578 & 48.6 \\
hCA VI $^{c}$ & 35.7 & $>1000$ & 61.2 \\
hCA VII $^{c}$ & 27.9 & $>1000$ & 9.1 \\
hCA IX $^{d}$ & 54.5 & $>1000$ & 767 \\
hCA XII $^{d}$ & 48.6 & $>1000$ & 167.4 \\
mCA XIII & 7.9 & $>1000$ & 6.0 \\
hCA XIV $^{c}$ & 7.8 & $>1000$ & 9.7
\end{tabular}

${ }^{a}$ Data were obtained by ref $298 .{ }^{b} \mathrm{~h}=$ human, $\mathrm{m}=$ murine isozyme. ${ }^{c}$ Full length enzyme. ${ }^{d}$ Catalytic domain. ${ }^{e}$ Preincubation of $6 \mathrm{~h}$ between enzyme and inhibitor.

shown to inhibit all the investigated CA isoforms, with inhibition constants ranging from nanomolar to millimolar. To understand this peculiar inhibition profile, the two coumarins 137 and 138 were cocrystallized with hCA II. ${ }^{298,299}$ Surprisingly, the analysis of the electron density maps in both complexes did not show the binding of such compounds in the enzyme active site, but evidenced the binding of their hydrolysis products, namely the cis-2-hydroxy- 
4-(1S-3-methylbutyl)-3-methoxy-cinnamic acid 140 and trans2-hydroxy-cinnamic acid 141, for 137 and 138, respectively, to the CA II active site. These data were explained assuming that the zinc-bound hydroxide anion, responsible for the various catalytic activities of CAs, ${ }^{1,2,5}$ among which the esterase activity, ${ }^{333}$ hydrolyzes the lactone ring of $\mathbf{1 3 7}$ and $\mathbf{1 3 8}$ leading to the formation of the corresponding 2-hydroxy-cinnamic acids (see Scheme 4). The obtained hydrolysis products did not

Scheme 4. CA-Catalyzed Hydrolysis of Coumarins 137 and 138 to the Corresponding 2-Hydroxy-cinnamic acids 140 and 141

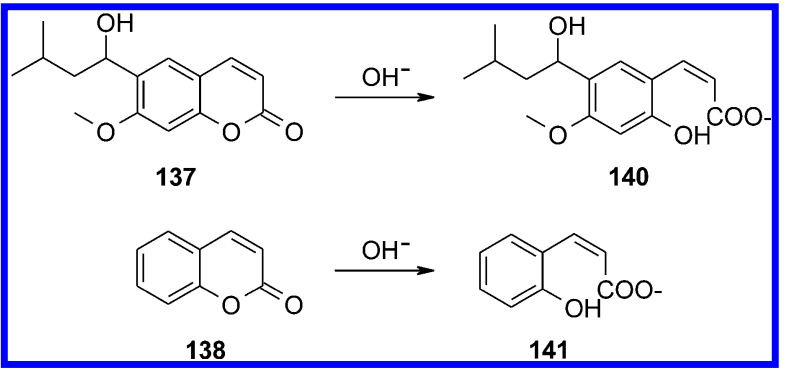

leave the active site but remained bound to its entrance, partially occluding it, stabilized by a large number of hydrophobic and polar interactions (Figure 51). This suicide inhibition mechanism was then confirmed by several kinetic and mass spectrometry experiments. ${ }^{298}$

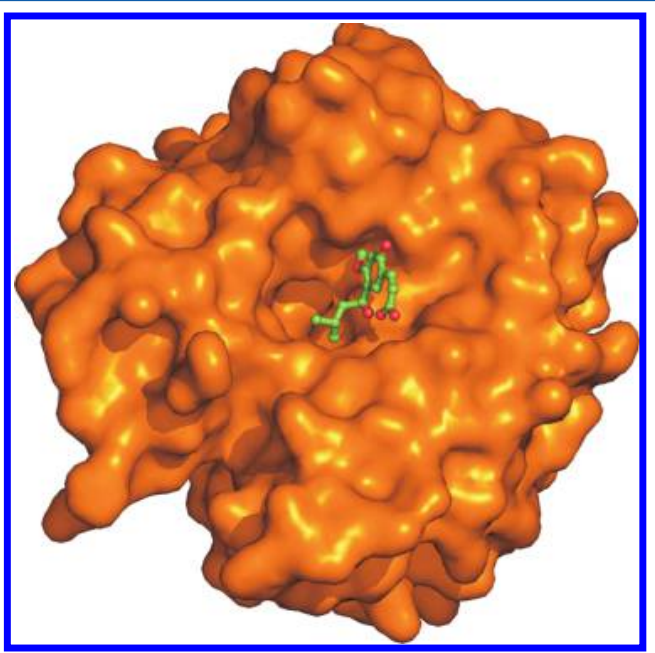

Figure 51. Solvent accessible surface of hCA II/140 adduct (PDB code $3 \mathrm{~F} 8 \mathrm{E}$ ) showing the inhibitor position at the entrance of the enzyme active site.

Altogether these data showed that coumarins constitute a totally new class of CAIs, with a novel inhibition mechanism. The unusual binding mode of these compounds makes their scaffolds a very promising starting point to develop nonclassical CAIs with more isoform selectivity. In particular, as coumarins bind to the entrance of the active site cavity, which is the region with a minor number of conserved residues among the different CAs, this binding mode offers the possibility to design inhibitors with improved selectivity with respect to the already existing CAIs. Indeed, several studies ${ }^{334,335}$ evidenced a rather large number of coumarin derivatives possessing CA IX, CA XII, CA XIII, and other CA isoform-selective behavior. Of great importance is the discovery of highly selective CA IX/XII

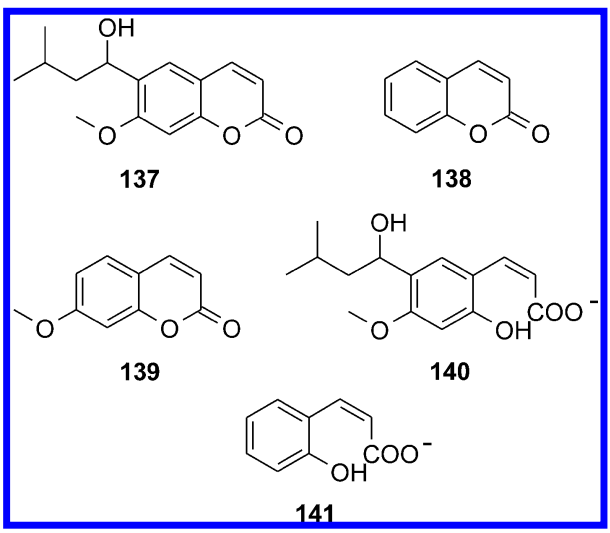

inhibitors belonging to the coumarin class, which do not significantly inhibit CA I and II. ${ }^{334,335}$ One such compound, a glycosyl-substituted coumarin, showed excellent antitumor and antimetastatic effects in an animal model of breast cancer and has been further evaluated in preclinical models of this disease. $^{336}$

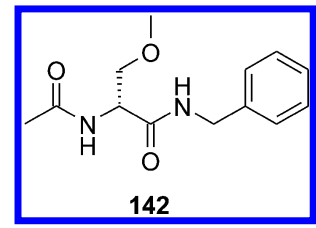

(2R)-2-Acetylamino- $N$-benzyl-3-methoxypropionamide 142 (known as lacosamide) is an antiepileptic drug (discovered by Kohn's group), ${ }^{337-343}$ which possesses some analogies with coumarins when binding to the CA II active site. Indeed, a recently reported inhibition study showed that lacosamide inhibits mammalian CAs I-XV with inhibition constants in range of $331 \mathrm{nM}$ to $4.56 \mu \mathrm{M}^{300}$ Moreover, its crystallographic structure in complex with CA II reveals that lacosamide binds to CA II, being located in the same site where coumarins were found (Figure 52), making favorable van der Waals interactions with Ile91, Gln92, Val121, Phe131, and Leu198. Again, as observed for coumarins no interactions with the $\mathrm{Zn}^{2+}$ catalytic

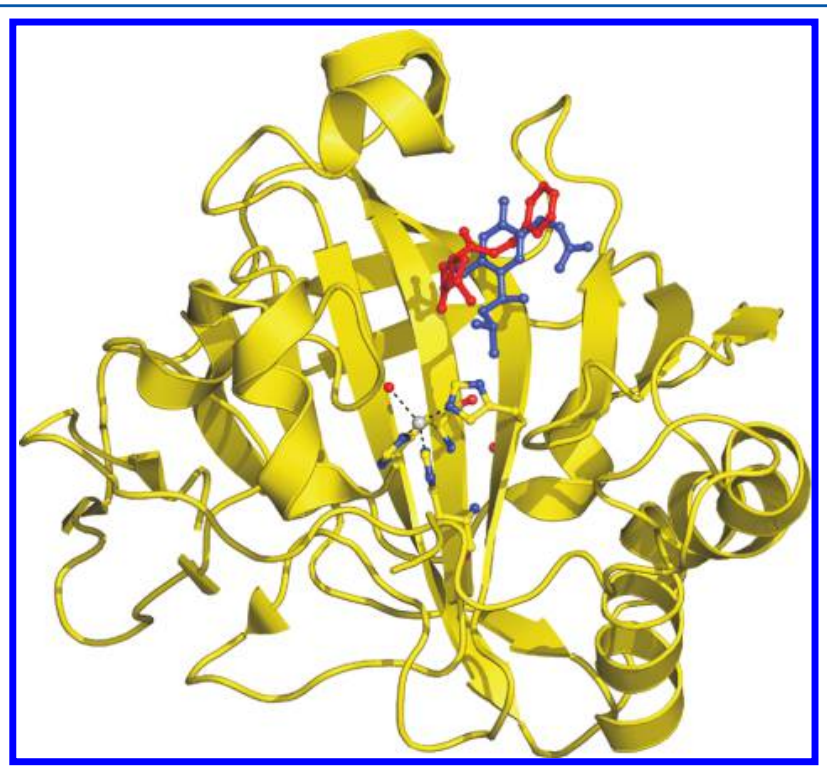

Figure 52. Superposition of hCA II/inhibitor adducts: $\mathbf{1 4 0}$ is reported in blue and 142 (PDB code 3IEO) in red. $\mathrm{Zn}^{2+}$ coordination is also shown. hCA II is represented as ribbon diagram. 
ion were evidenced. ${ }^{300}$ Such findings indicate that the coumarin-binding site may accommodate structurally diverse compounds which possess an inhibition mechanism distinct of that of sulfonamides, opening new perspectives for designing new CAIs with various biomedical applications.

\section{CONCLUSIONS}

A large amount of X-ray structural data have been accumulated in the last years for adducts of CAs with the main classes of inhibitors: the pharmacologically relevant sulfonamides and their isosteres (sulfamates and sulfamides), ureates and hydroxamates, the simple inorganic anions, phenols, polyamines, and very recently, the totally new class of inhibitors, the coumarins/thiocoumarins. However, although X-ray crystal structures are already available for the majority of the twelve catalytically active members of the human CA family, most of the reported complexes involves isozyme II. A careful analysis of these data allowed to divide CA inhibitors in two main classes: those that bind the enzyme active site anchoring themselves to the catalytic zinc ion, and those that are bound in the active site but do not interact directly with the metal ion.

The inhibitors belonging to the first class, the most studied ones from a structural point of view, are generally characterized by three main structural elements: a ZBG, an organic scaffold, and one or more "tails". The ZBGs leading to very potent CAIs may belong to various functionalities, with the classical sulfonamide one still constituting the main player. However, bioisosteres of the $\mathrm{SO}_{2} \mathrm{NH}_{2}$ moiety, such as the sulfamate and sulfamide groups, were shown to lead to inhibitors equipotent, and in some cases even stronger than the corresponding sulfonamides. Several others ZBGs, such as unsubstituted hydroxamates, $N$-hydroxy-urea and urea, have also been explored in detail by means of crystallographic methods and can be considered as promising alternatives.

A large number of organic scaffolds were also investigated, with four main groups of compounds evidenced so far: aromatic, heterocyclic, aliphatic and sugar derivatives. Aromatic (most of the time benzene or naphthalene derivatives) sulfonamides constitute by far the most extensively investigated class of CAIs. The heterocyclic sulfonamides are immediately second in importance to the benzenesulfonamides, considering the fact that they are contained in many of the clinically used CA inhibitors. Many of these CAIs incorporate 5-membered sulfur-containing heterocycles (thiophene, 1,3,4-thiadiazole, 1,3,4-thiadiazoline) or annulated 5-membered such rings (benzo-[b]-thiophene, benzo-thiazine, etc.). Several aliphatic derivatives were also investigated, such as the clinically used antiepileptic zonisamide, and some 1,3,4-thiadiazole derivatives substituted with $\mathrm{CH}_{2} \mathrm{SO}_{2} \mathrm{NH}_{2}$ or $\mathrm{CF}_{2} \mathrm{SO}_{2} \mathrm{NH}_{2}$ moieties. The binding modes and selectivity profiles for inhibition of mammalian CAs by these compounds are totally new and different from those of the aromatic/heterocyclic sulfonamides investigated earlier. Thus, more detailed investigations of the aliphatic derivatives as CAIs, eventually incorporating other ZBGs than the sulfonamide one, are highly desirable and may lead to important advances. Finally several sugar derivatives, incorporating both sulfonamide, sulfamate and sulfamide ZBGs, were also characterized by means of X-ray crystallography, leading to very interesting results.

However, the main problem with CAIs incorporating only the ZBG and the organic scaffold, was related to the fact that they were promiscuous inhibitors of all (or most of the) hCAs, since the interactions of the ZBGs and the organic scaffold with the enzyme were basically the same between the inhibitors and most CA isozymes. For this reason in 1999 Supuran's group developed the so-called "tail-approach", which afforded the rather simple synthesis of a large number of CAIs starting from aromatic/heterocyclic scaffolds. In this approach various moieties (tails) were introduced by normal chemical modification reactions (acylation, alkylation, arylsulfonylation, condensation, etc.) in the aromatic/heterocyclic scaffold. In this way, it was possible both to modulate the physicochemical properties of the synthesized inhibitors, such as water solubility, lipophilicity, membrane impermeability etc., and their affinity to the various isozymes. These novel generation inhibitors showed much more interesting inhibition profiles as compared to the classical ones. Both X-ray crystal structures with isoform II and homology models with other relevant isoforms are available for some of these compounds, proving that both favorable interactions as well as clashes with particular amino acids, are critical for the inhibition profile and isozyme selectivity issues.

The main problem with this type of inhibitors resides in the predominant role played by the ZBG in the interaction with CA active site. As a consequence, any change in the thermodynamics of binding caused by the nature of the organic scaffold and/or the tail may have only a small effect on the enzymeinhibitor affinity, and consequently requires a very careful experimental design. For this reason, the last years have seen the development of the second class of inhibitors, among which phenols, ${ }^{295,296}$ polyamines, ${ }^{297}$ coumarins/thiocoumarins, and the antiepileptic drug lacosamide ${ }^{298,299}$ are the most interesting examples. These molecules are bound in the active site but do not interact directly with the catalytic zinc ion. In particular, phenols and polyamines are anchored to the zinc-coordinated water molecule/hydroxide ion, while the coumarins and lacosamide bind to the border of the active cavity partially occluding its entrance. Thus, over the years, starting from the zinc binders, novel generations of inhibitors were observed to bind further and further away from the zinc. This is of great interest since the regions of the active site, which are less conserved among the different isoforms are those at the entrance of the cavity. This also explains why inhibitors which bind to these regions show much better degrees of isoform selectivity, and often inhibit strongly only one, or a restricted number of isoforms, among the fifteen presently described in humans.

Altogether these studies have demonstrated that, even though much work must be still done, important progresses on the knowledge of factors governing selective inhibition have been obtained. Indeed, some examples of isozyme-specific inhibitors are already available and are described in the concluding part of this review.

Spermine ${ }^{297} 133$ is a polyamine which behaves as a CA IVhighly selective inhibitor. In fact, it is a low nanomolar inhibitor only of CA IV ( $K_{\mathrm{I}}$ of $10 \mathrm{nM}$ ) whereas its inhibition of other isoforms is in the micromolar range. ${ }^{297}$ As the physiological role of CA IV in many tissues is not known exactly, spermine and some of its congeners may constitute important tools to unravel the precise function of this extracellular isoform.

Recently two different examples of CA VA/VB-selective inhibitors have also been reported. The first example is constituted by some naturally occurring phenols of types 132 and 143-147, which show nanomolar inhibition of the isoforms hCA VA and $\mathrm{VB}$, and only micromolar affinity for the offtarget one hCA I and II. ${ }^{344,345}$ The second example is represented by a class of highly lipophilic sulfonamides 


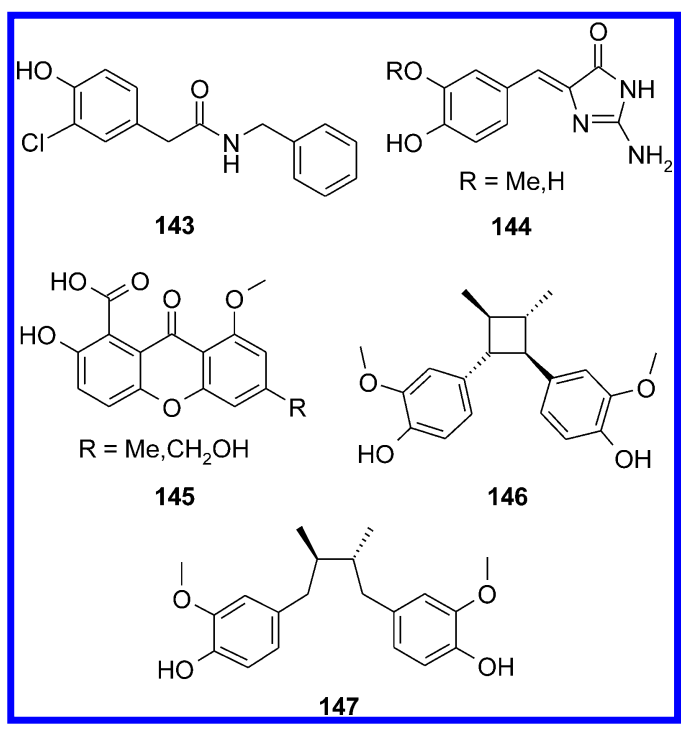

incorporating $R$ - or $S$-camphorsulfonyl moieties. ${ }^{346}$ Indeed some of these derivatives, such as 148 , show the following inhibition constants: $K_{\mathrm{I}}(\mathrm{CA} \mathrm{I})=5246 \mathrm{nM}, K_{\mathrm{I}}(\mathrm{CA}$ II $)=1773$ $\mathrm{nM}, K_{\mathrm{I}}(\mathrm{CA} \mathrm{VA})=5.9 \mathrm{nM}$, and $K_{\mathrm{I}}(\mathrm{CA} \mathrm{VB})=7.8 \mathrm{nM} \cdot{ }^{346}$ This is the most mitochondrial isoform-selective sulfonamide CAI reported to date.

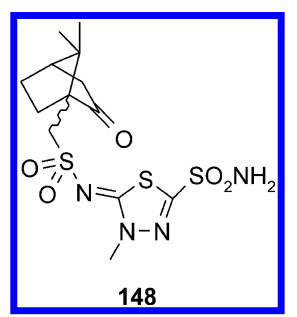

A series of low nanomolar and subnanomolar CA VI inhibitors was also reported, ${ }^{347}$ belonging to the glycosylamine-sulfanilamide class of which compound 130 is an example. The glucose, ribose, arabinose, xylose, and fucose derivatives show excellent CA VI inhibitory activity, with $\mathrm{K}_{\mathrm{I}} \mathrm{s}$ in the range of $0.56-5.1 \mathrm{nM}$, whereas the least active derivatives, incorporating gallactose, mannose, and rhamnose scaffolds have inhibition constants in the range of 10.1-34.1 nM. Many of these sulfonamides are also selective inhibitors for their interaction with CA VI over the physiologically dominant and ubiquitous isoform CA II, with selectivity ratios of 4.11-35.93 for inhibiting the secreted over the cytosolic isozyme. Because of their high water solubility and high affinity for CA VI over CA II, these compounds might be useful tools for better understanding the secreted isoform CA VI.

The first series of CA VII-selective sulfonamide CAIs was reported by Guzel et al. $^{348}$ Indeed, derivatives of types 149152, incorporating aromatic or heterocyclic sulfonamide heads and the aryl- $\mathrm{CH}_{2} \mathrm{CONH}$ moieties show low nanomolar inhibitory activity against hCA VII $\left(\mathrm{K}_{\mathrm{I}} \mathrm{s}\right.$ in the range of 4.7$8.5 \mathrm{nM}$, and selectivity ratios for inhibiting CA VII over CA II in the range of 8.6-49.6). Thus, the advent of such CA VIIselective inhibitors has opened the possibility of being able to establish soon the involvement of CA VII both in epileptogenesis and neuropathic pains. ${ }^{349}$

Important results have been obtained also with isozymes CA IX and XII, which, as described in the Introduction, are predominantly found in tumor cells. Several approaches have been reported in the last years for obtaining compounds that

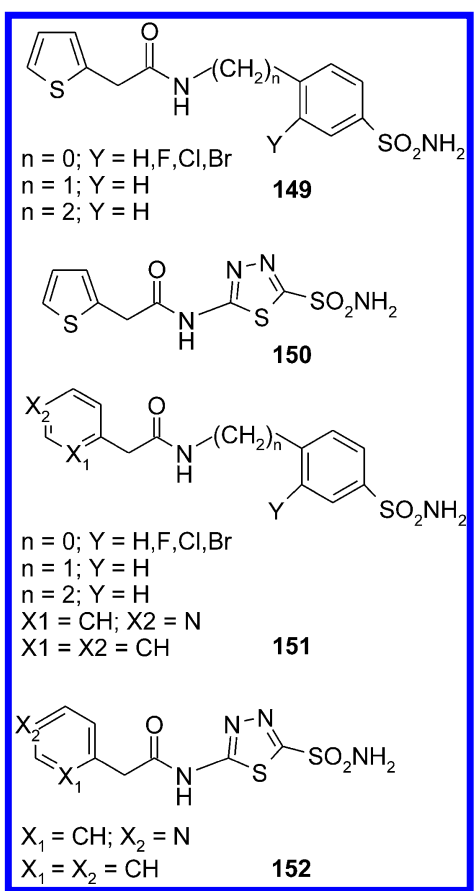

specifically target such isoforms among which: (i) fluorescent sulfonamides (such as compounds $\mathbf{4 2}$ discussed in this review), used for imaging purposes and for determining the role of CA IX in tumor acidification; ${ }^{151}$ (ii) positively (such as compounds 29-31 discussed in the review) or negatively charged compounds, which cannot cross plasma membranes due to their charged character and thus inhibit selectively only extracellular CAs, among which CA IX and XII; ${ }^{150}$ (iii) hypoxia-activatable compounds, which exploit the reducing conditions of hypoxic tumors to convert an inactive prodrug into an active inhibitor; ${ }^{185}$ (iv) sugar-containing sulfonamides/ sulfamates/sulfamides, which due to their highly hydrophilic character do not easily cross membranes and thus possess an enhanced affinity for extracellular CAs; ${ }^{275}$ (v) nanoparticles coated with CAIs. ${ }^{162}$

However, the most important perspectives in the drug design of CA IX selective inhibitors stemmed only recently from the crystal structure resolution of the enzyme catalytic domain, ${ }^{63}$ which highlighted two unique features of this enzyme: its dimeric nature with both active sites exposed on the same side of the dimer and the location of the proteoglycan-like (PG) domain at the border of the active site cleft. Indeed, it is imaginable that "two-prong" inhibitors, able to target at the same time either both active sites or the active site and the PG domain, could represent an interesting alternative to classical CA inhibitors, which target only the active site.

Moreover, the structural comparison of CA IX with other membrane associated CAs highlighted important differences in the region of amino acids 125-137, located at the border of the active site cavity, making this region an interesting target to address in the rational drug design of selective inhibitors. These findings are well in agreement with the observation of a good degree of selectivity showed by the most recently reported family of CAIs, that is, coumarins, ${ }^{298}$ which were shown to bind at the border of the active site close to this region. In particular, some studies, which reported a large series of derivatives (compounds 139 and 153-162) possessing various moieties substituting the (thio)coumarin ring, ${ }^{299,334,335}$ led to indentify some truly CA IX-selective inhibitors. For example compound 
160c was shown to be a low nanomolar inhibitor of only CA IX $\left(K_{\mathrm{I}}\right.$ of $\left.48 \mathrm{nM}\right)$, whereas it inhibited in the micromolar range all other twelve CAs, a feature never evidenced before for a sulfonamide CAI.

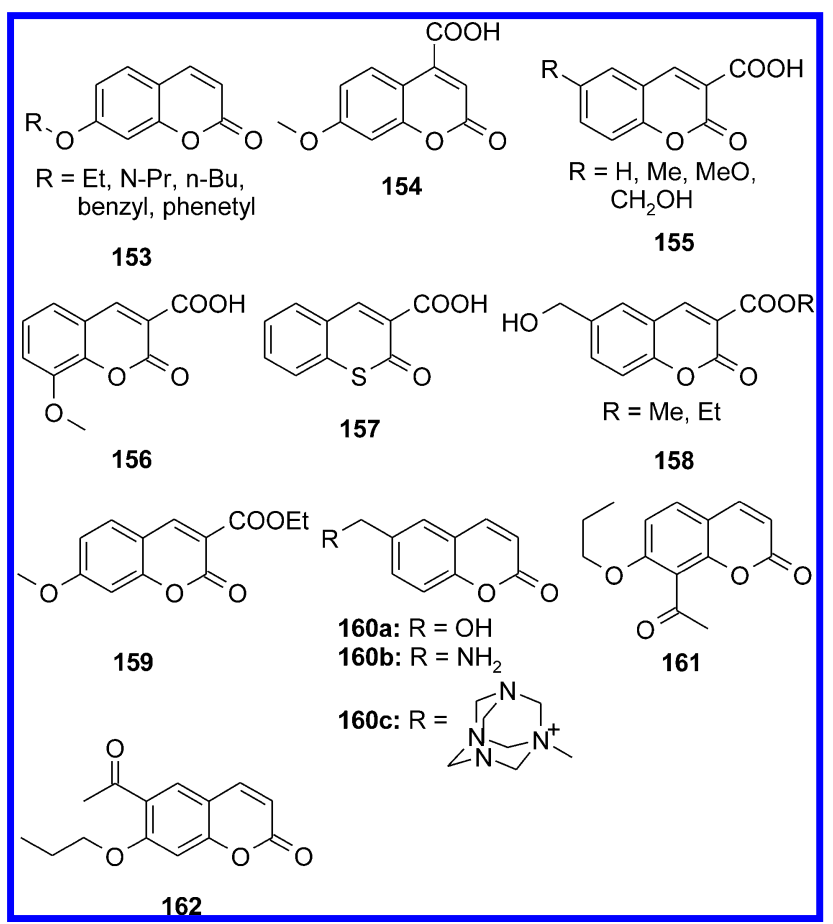

But the real breakthrough in the investigations of CAIs as antitumor agents has been only very recently published. ${ }^{336,350}$ In particular, it has been reported that both sulfonamidic (such as the ureido derivative 26) and coumarinic (such as the glycosyl coumarin 163) CA IX inhibitors strongly inhibit the growth of both primary tumors and metastases in several animal models of breast cancer. ${ }^{336,350}$ These data undoubtfully demonstrate the potential of CA IX inhibition for designing antitumor/antimetastatic agents possessing a novel mechanism of action with respect to the existing drugs.

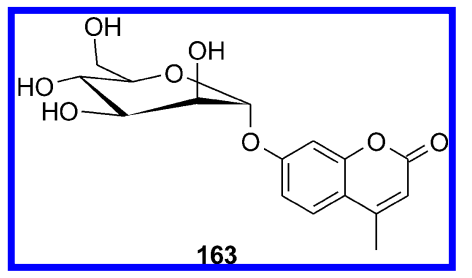

Unfortunately no isoform selective compounds have been described so far for CA XIII and CA XIV, except some coumarins which are CA XIII-selective. ${ }^{334,335}$

But what about CA I and II? Are there isoform selective inhibitors for them? The answer is yes, and again the coumarins provide the best examples of CA I and II-selective compounds. The natural product coumarin $\mathbf{1 3 7}$ was a nanomolar CA I and II inhibitor but inhibited all other isoforms only in the high micromolar range. The simple, unsubstituted coumarin 138 is also a similar case, showing low micromolar inhibition of CA I and II, and a millimolar inhibition of isoforms CA III-CA XV (see Table 6). All these data clearly show that it may be possible to obtain CAIs with selectivity for each of human isoforms, although this field is very new. We conclude the review stressing the fact that the family of CAs, with their large number of isoforms, provides an excellent scientific example of successful drug design of selective inhibitors for structurally very similar proteins. With their multiple medicinal chemistry applications, such isoform-selective compounds may lead to novel drugs with less side effects compared to the present ones in a wide range of diseases.

\section{AUTHOR INFORMATION}

\section{Corresponding Author}

*Phone: +39-055-4573005 (C.T.S.); +39-081-2534579 (G.D.S.). Fax: +39-055-4573385 (C.T.S.); +39-081-2536642 (G.D.S.). E-mail: claudiu.supuran@unifi.it (C.T.S.); gdesimon@unina.it (G.D.S.).

Notes

The authors declare no competing financial interest.

\section{Biographies}

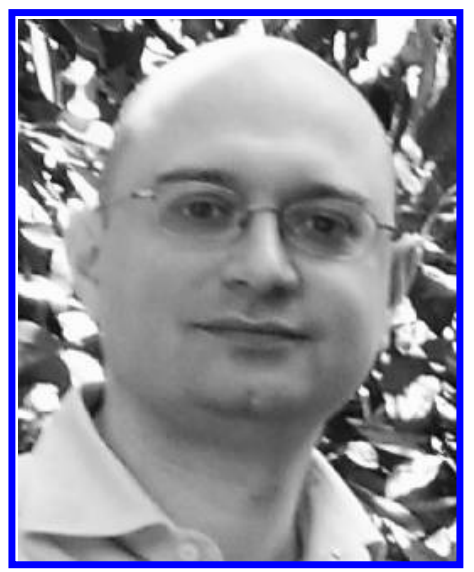

Vincenzo Alterio was born in 1975 in Naples, Italy. He graduated in Chemistry at the University of Naples "Federico II" in 2001 and received his Ph.D. in Chemistry at the same University in 2004. From 2005 to 2008, he carried out his postdoctoral activity at the Institute of Biostructures and Bioimaging (IBB) of the Italian National Research Council. In 2006, he was guest researcher at the Biochemistry Institute of the University of Luebeck (Germany). Since 2009, he is researcher at the Institute of Biostructures and Bioimaging. His main research interests are focused on the structural characterization by means of $\mathrm{X}$ ray crystallography of proteins and protein-ligand complexes. In particular, his attention is mainly oriented to the study of biological systems having medical or biotechnological interest.

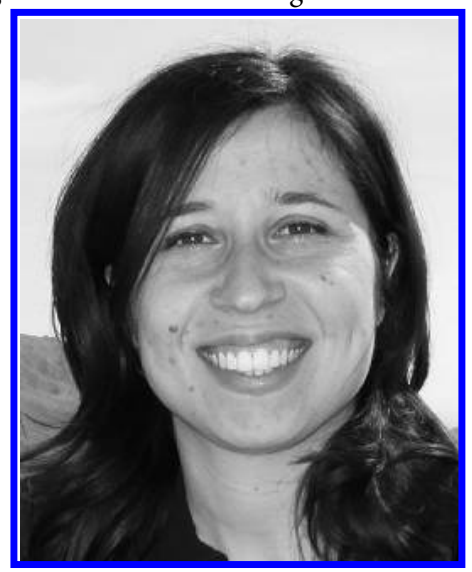

Anna Di Fiore received her B.S. in Chemistry in 2001 from the University of Naples "Federico II", Italy, and Ph. D. in Chemistry at the same university in 2004 . She is currently a postdoctoral fellow at 
Institute of Biostructures and Bioimaging-CNR in Naples, Italy. Her research interests include structural and functional characterization of proteins playing key role in several physiological and physiopathological processes. In particular, her activities consists of the crystallization and threedimensional structure determination by X-ray diffraction of proteins and protein-inhibitor complexes.

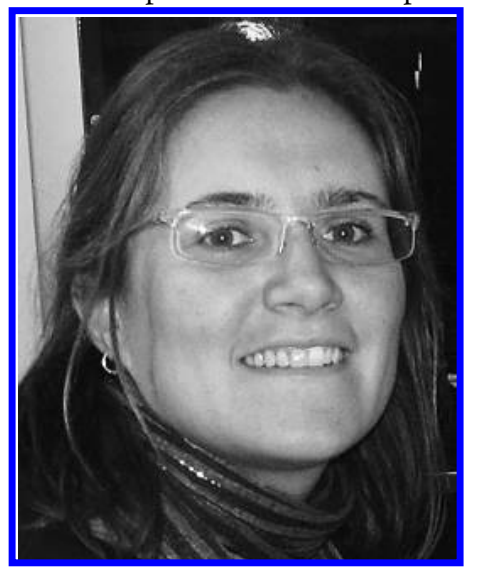

Katia D'Ambrosio obtained her BS in Chemistry and Pharmaceutical Technology at the University of Naples "Federico II" in 2000. She was visiting student at the Pharmaceutical Chemistry Department at the University of Montpellier and guest investigator at the "LCM3B" laboratory at the University "Henry Poincairè" in Nancy. In 2004, she received her $\mathrm{PhD}$ in Chemistry of Biological Processes at the University of Naples "Federico II". Since 2009, she is Researcher at the Institute of Biostructures and Bioimaging of the Italian National Research Council. Her research interests are in the area of structural characterization of protein and protein-ligand complexes by means of $\mathrm{X}$-ray crystallography. In particular, she worked on different proteins involved in oxidoreduction mechanisms and enzymes responsible for some human pathologies.

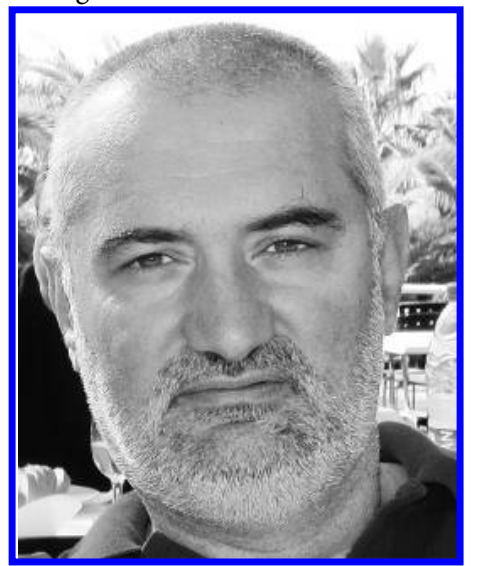

Claudiu T. Supuran received his BSc in chemistry from the Polytechnic University of Bucharest, Romania (1987) and $\mathrm{PhD}$ in chemistry at the same university in 1991. In 1990 he became assistant and then associate professor of chemistry at the University of Bucharest. He was a visiting scholar at University of Florida, Gainesville, USA, at Grifitth University, Brisbane, Australia and a visiting professor at University of La Plata, Argentina. In 1995 he moved at the University of Florence, Italy, where he is currently research fellow and contract professor of chemistry. His main research interests include medicinal chemistry, design of enzyme inhibitors and activators, heterocyclic chemistry, chemistry of sulfonamides, sulfamates and sulfamides, biologically active organoelement derivatives, QSAR studies, X-ray crystallography of metallo-enzymes, metal complexes with biologically active ligands (metal-based drugs), carbonic anhydrases, cyclooxygenases, serine proteases, matrix metalloproteinases, bacterial proteases, and amino acid derivatives among others. He has published more than 700 papers in these fields.

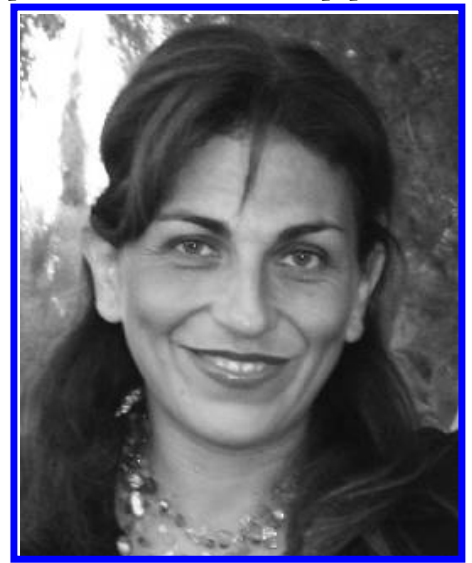

Giuseppina De Simone was born in 1970 in Naples, Italy. She graduated in chemistry at the University of Naples Federico II (1993). She worked from August 1994 to January 1995 at the Drug Design Group"-Sandoz Pharma AG" in Basel, and from September 1995 to February 1996 at the University of Pavia. From November 1996 to January 1997 she was visiting scientist at the Max Plank InstituteHamburg Germany. In 1998 she received her PhD at the University of Naples. Since 2003, she is Senior Researcher at the Institute of Biostructures and Bioimaging (IBB) of the Italian National Research Council. Her main research interests include X-ray crystallography of macromolecules, structure based drug design, carbonic anhydrases, serine proteases, esterases/lipases, peptide nucleic acids. She has published more than 80 papers in these fields.

\section{ACKNOWLEDGMENTS}

Research form our groups was financed by several EU projects (DeZnIt, Euroxy and Metoxia) from the 6th and 7th framework programme.

$\begin{array}{ll}\text { ABBREVIATIONS } \\ \text { CA } & \text { carbonic anhydrase } \\ \text { CA I } & \text { isoform I of CA } \\ \text { CA II } & \text { isoform II of CA, etc } \\ \mathrm{h} & \text { human } \\ \mathrm{m} & \text { murine } \\ \mathrm{b} & \text { bovine } \\ \text { CAI } & \text { CA inhibitor } \\ \text { hCA } & \text { human CA } \\ \text { CARP } & \text { CA-related protein } \\ \text { ITC } & \text { isothermal titration calorimetry } \\ \text { EPR } & \text { Electron paramagnetic resonance } \\ \text { TEMPO } & \text { 2,2,6,6-tetramethylpiperidine-1-oxyl } \\ \text { MRI } & \text { magnetic resonance imaging } \\ \text { PG } & \text { proteoglycan-like }\end{array}$

ESI-FTICR-MS electrospray ionization Fourier transform ion cyclotron resonance mass spectrometry

ZBG zinc-binding group

STS steroid sulfatase

DASI dual aromatase-steroid sulfatase inhibitor

\section{REFERENCES}

(1) Krishnamurthy, V. M.; Kaufman, G. K.; Urbach, A. R.; Gitlin, I.; Gudiksen, K. L.; Weibel, D. B.; Whitesides, G. M. Chem. Rev. 2008, 108, 946.

(2) Supuran, C. T. Nat. Rev. Drug Discovery 2008, 7, 168. 
(3) Drug Design of Zinc-Enzyme Inhibitors: Functional, Structural, and Disease Applications; Supuran, C. T., Winum, J.-Y., Eds.; Wiley: Hoboken, NJ, 2009; Part II.

(4) Xu, Y.; Feng, L.; Jeffrey, P. D.; Shi, Y.; Morel, F. M. Nature 2008, 452, 56.

(5) Carbonic Anhydrase: Its Inhibitors and Activators; Supuran, C. T., Scozzafava, A., Conway, J., Eds.; CRC Press: Boca Raton, FL, 2004.

(6) Maren, T. H. Physiol. Rev. 1967, 47, 595.

(7) Lane, T. W.; Saito, M. A.; George, G. N.; Pickering, I. J.; Prince, R. C.; Morel, F. M. Nature 2005, 435, 42.

(8) Tripp, B. C.; Smith, K.; Ferry, J. G. I. Biol. Chem. 2001, 276, 48615

(9) Ferry, J. F. Biochim. Biophys. Acta 2010, 1804, 374.

(10) Clare, B. W.; Supuran, C. T. Expert Opin. Drug Metab. Toxicol. 2006, 2, 113.

(11) Lindskog, S. Pharmacol. Ther. 1997, 74, 1.

(12) Supuran, C. T. Curr. Top. Med. Chem. 2007, 7, 825.

(13) Supuran, C. T.; Scozzafava, A.; Casini, A. Med. Res. Rev. 2003, 23, 146.

(14) Supuran, C. T.; Scozzafava, A. Bioorg. Med. Chem. 2007, 15, 4336.

(15) Pastorekova, S.; Parkkila, S.; Pastorek, J.; Supuran, C. T. I. Enzyme Inhib. Med. Chem 2004, 19, 199.

(16) Scozzafava, A.; Mastrolorenzo, A.; Supuran, C. T. Expert. Opin. Ther. Pat. 2006, 16, 1627.

(17) Scozzafava, A.; Mastrolorenzo, A.; Supuran, C. T. Expert Opin. Ther. Pat. 2004, 14, 667.

(18) Winum, J. -Y.; Scozzafava, A.; Montero, J. -L.; Supuran, C. T. Mini Rev. Med. Chem. 2006, 6, 921.

(19) Temperini, C.; Scozzafava, A.; Supuran, C. T. In Drug Desion of Zinc-Enzyme Inhibitors: Functional, Structural, and Disease Applications: Supuran, C. T., Winum, J.-Y., Eds.; Wiley: Hoboken, NJ, 2009; p 473.

(20) De Simone, G.; Di Fiore, A.; Supuran, C. T. Curr. Pharm. Des. 2008, 14, 655 .

(21) De Simone, G.; Supuran, C. T. Curr. Top. Med. Chem. 2007, 7, 879.

(22) Supuran, C. T.; Di Fiore, A.; De Simone, G. Expert Opin. Emerg. Drugs 2008, 13, 383.

(23) Winum, J.-Y.; Rami, M.; Scozzafava, A.; Montero, J.-L.; Supuran, C. T. Med. Res. Rev. 2008, 28, 445.

(24) Guler, O. O.; De Simone, G.; Supuran, C. T. Curr. Med. Chem. 2010, 17, 1516.

(25) Thiry, A.; Dogné, J. M.; Supuran, C. T.; Masereel, B. Curr. Pharm. Des. 2008, 14, 661.

(26) Hen, N.; Bialer, M.; Yagen, B.; Maresca, A.; Aggarwal, M.; Robbins, A. H.; McKenna, R.; Scozzafava, A.; Supuran, C. T. I. Med. Chem. 2011, 54, 3977.

(27) De Simone, G.; Supuran, C. T. Biochim. Biophvs. Acta 2010, 1804, 404.

(28) Gao, B. B.; Clermont, A.; Rook, S.; Fonda, S. J.; Srinivasan, V. J.; Wojtkowski, M.; Fujimoto, J. G.; Avery, R. L.; Arrigg, P. G.; Bursell, S. E.; Aiello, L. P.; Feener, E. P. Nat. Med. 2007, 13, 181.

(29) Mincione, F.; Scozzafava, A.; Supuran, C. T. Curr. Pharm. Des. 2008, 14, 649 .

(30) Supuran, C. T. Curr. Pharm. Des. 2008, 14, 641.

(31) De Simone, G.; Scozzafava, A.; Supuran, C. T. Chem. Biol. Drug Des. 2009, 74, 317.

(32) Basnyat, B.; Gertsch, J. H.; Johnson, E. W.; Castro-Marin, F.; Inoue, Y.; Yeh, C. High Alt. Med. Biol. 2003, 4, 45.

(33) Swenson, E. R.; Teppema, L. J. L. Appl. Phvsiol. 2007, 102, 1305.

(34) Barreiro, E.; Hussain, S. N. A. Antioxid. Redox Signal. 2010, 12, 417.

(35) Brancaccio, P.; Lippi, G.; Maffulli, N. Clin. Chem. Lab. Med. 2010, 48, 757 .

(36) Zimmerman, U. J.; Wang, P.; Zhang, X.; Bogdanovich, S.; Forster, R. IUBMB Life. 2004, 56, 343.

(37) Matsui, H.; Murakami, M.; Wynns, G. C.; Conroy, C. W.; Mead, A.; Maren, T. H.; Sears, M. L. Exp. Eve Res. 1996, 62, 409.
(38) Datta, R.; Waheed, A.; Bonapace, G.; Shah, G. N.; Sly, W. S. Proc. Natl. Acad. Sci. U.S.A. 2009, 106, 3437.

(39) Ochrietor, J. D.; Clamp, M. F.; Moroz, T. P.; Grubb, J. H.; Shah, G. N.; Waheed, A.; Sly, W. S.; Linser, P. I. Exp. Eve Res. 2005, 81, 492. (40) Tang, Y.; Xu, H.; Du, X.; Lit, L.; Walker, W.; Lu, A.; Ran, R.; Gregg, J. P.; Reilly, M.; Pancioli, A.; Khoury, J. C.; Sauerbeck, L. R.; Carrozzella, J. A.; Spilker, J.; Clark, J.; Wagner, K. R.; Jauch, E. C.; Chang, D. J.; Verro, P.; Broderick, J. P.; Sharp, F. R. I. Cereb. Blood Flow Metab. 2006, 26, 1089.

(41) De Simone, G.; Supuran, C. T. In Drug Design of Zinc-Enzyme Inhibitors: Functional, Structural, and Disease Applications; Supuran, C. T., Winum, J.-Y., Eds.; Wiley: Hoboken, NJ, 2009; p 241.

(42) Nishimori, I.; Minakuchi, T.; Onishi, S.; Vullo, D.; Scozzafava, A.; Supuran, C. T. I. Med. Chem. 2007, 50, 381.

(43) Kivelä, J.; Parkkila, S.; Parkkila, A. K.; Rajaniemi, H. Caries Res. 1999, 33, 178.

(44) Ruusuvuori, E.; Li, H.; Huttu, K.; Palva, J. M.; Smirnov, S.; Rivera, C.; Kaila, K.; Voipio, J. I. Neurosci. 2004, 24, 2699.

(45) Aspatwar, A.; Tolvanen, M. E.; Ortutay, C.; Parkkila, S. Curr. Pharm. Des. 2010, 16, 3264.

(46) Nishimori, I. In Carbonic Anhvdrase: Its Inhibitors and Activators; Supuran, C. T., Scozzafava, A., Conway, J., Eds.; CRC Press: Boca Raton, FL, 2004; p 25.

(47) Pastorekova, S.; Parkkila, S.; Zavada, J. Adv. Clin. Chem. 2006, $42,167$.

(48) Battke, C.; Kremmer, E.; Mysliwietz, J.; Gondi, G.; Dumitru, C.; Brandau, S.; Lang, S.; Vullo, D.; Supuran, C. T; Zeidler, R. Cancer Immunol. Immunother. 2011, 60, 649.

(49) Liao, S. Y.; Ivanov, S.; Ivanova, A.; Ghosh, S.; Cote, M. A.; Keefe, K.; Coca-Prados, M.; Stanbridge, E. J.; Lerman, M. I. I. Med. Genet. 2003, 40, 257.

(50) Lehtonen, J.; Shen, B.; Vihinen, M.; Casini, A.; Scozzafava, A.; Supuran, C. T.; Parkkila, A. K.; Saarnio, J.; Kivelä, A. J.; Waheed, A.; Sly, W. S.; Parkkila, S. I. Biol. Chem. 2004, 279, 2719.

(51) Shah, G. N.; Ulmasov, B.; Waheed, A.; Becker, T.; Makani, S.; Svichar, N.; Chesler, M.; Sly, W. S. Proc. Natl. Acad. Sci.U.S.A. 2005, 102, 16771.

(52) Ogilvie, J. M.; Ohlemiller, K. K.; Shah, G. N.; Ulmasov, B.; Becker, T. A.; Waheed, A.; Hennig, A. K.; Lukasiewicz, P. D.; Sly, W. S. Proc. Natl. Acad. Sci. U.S.A. 2007, 104, 8514.

(53) Alterio, V.; Di Fiore, A.; D’Ambrosio, K.; Supuran, C. T.; De Simone, G. In Drug Design of Zinc-Enzyme Inhibitors: Functional, Structural, and Disease Applications; Supuran, C. T., Winum, J.-Y., Eds.; Wiley: Hoboken, NJ, 2009; p 73.

(54) Kannan, K. K.; Ramanadham, M.; Jones, T. A. Ann. N.Y. Acad. Sci. 1984, 429, 49.

(55) Eriksson, A. E.; Kylsten, P. M.; Jones, T. A.; Liljas, A. Proteins 1988, 4, 283.

(56) Eriksson, A. E.; Liljas, A. Proteins 1993, 16, 29.

(57) Boriack-Sjodin, P. A.; Heck, R. W.; Laipis, P. J.; Silverman, D. N.; Christianson, D. W. Proc. Natl. Acad. Sci. U.S.A. 1995, 92, 10949.

(58) Stams, T.; Nair, S. K.; Okuyama, T.; Waheed, A.; Sly, W. S.; Christianson, D. W. Proc. Natl. Acad. Sci. U.S.A. 1996, 93, 13589.

(59) Whittington, D. A.; Waheed, A.; Ulmasov, B.; Shah, G. N.; Grubb, J. H.; Sly, W. S.; Christianson, D. W. Proc. Natl. Acad. Sci. U.S.A. 2001, 98, 9545.

(60) Whittington, D. A.; Grubb, J. H.; Waheed, A.; Shah, G. N.; Sly, W. S.; Christianson, D. W. I. Biol. Chem. 2004, 279, 7223.

(61) Duda, D. M.; Tu, C.; Fisher, S. Z.; An, H.; Yoshioka, C.; Govindasamy, L.; Laipis, P. J.; Agbandje-McKenna, M.; Silverman, D. N.; McKenna, R. Biochemistry 2005, 44, 10046.

(62) Di Fiore, A.; Monti, S. M.; Hilvo, M.; Parkkila, S.; Romano, V.; Scaloni, A.; Pedone, C.; Scozzafava, A.; Supuran, C. T.; De Simone, G Proteins 2008, 74, 164.

(63) Alterio, V.; Hilvo, M.; Di Fiore, A.; Supuran, C. T.; Pan, P.; Parkkila, S.; Scaloni, A.; Pastorek, J.; Pastorekova, S.; Pedone, C.; Scozzafava, A.; Monti, S. M.; De Simone, G. Proc. Natl. Acad. Sci. U.S.A. 2009, 106, 16233. 
(64) Di Fiore, A.; Truppo, E.; Supuran, C. T.; Alterio, V.; Dathan, N.; Bootorabi, F.; Parkkila, S.; Monti, S. M.; De Simone, G. Bioorg. Med. Chem. Lett. 2010, 20, 5023.

(65) Pilka, E. S.; Kochan, G.; Oppermann, U.; Yue, W. W. Biochem. Biophys. Res. Commun. 2012, 419, 485.

(66) Håkansson, K.; Carlsson, M.; Svensson, L. A.; Liljas, A. I. Mol. Biol. 1992, 227, 1192.

(67) Stams, T.; Christianson, D. W. In The Carbonic Anhvdrases. New Horizons; Chegwidden, W. R., Carter, N. D., Edwards, Y. H., Eds.; Birkhäuser Verlag: Basel, Switzerland, 2000; p 159.

(68) Christianson, D. W.; Fierke, C. A. Acc. Chem. Res. 1996, $29,331$.

(69) Sjöblom, B.; Polentarutti, M.; Djinovic-Carugo, K. Proc. Natl. Acad. Sci. U.S.A. 2009, 106, 10609.

(70) Domsic, J. F.; Avvaru, B. S.; Kim, C. U.; Gruner, S. M.; Agbandje-McKenna, M.; Silverman, D. N.; McKenna, R. $\underline{\text { I. Biol. Chem. }}$ 2008, 283, 30766

(71) Merz, K. M., Jr. I. Am. Chem. Soc. 1991, 113, 406.

(72) Tu, C. K.; Silverman, D. N.; Forsman, C.; Jonsson, B. H.; Lindskog, S. Biochemistry 1989, 28, 7913.

(73) Silverman, D. N.; McKenna, R. Acc. Chem. Res. 2007, 40, 669.

(74) Roy, A.; Taraphder, S. L. Phys. Chem. B 2007, 111, 10563.

(75) Fisher, S. Z.; Maupin, C. M.; Budayova-Spano, M.; Govindasamy, L.; Tu, C. K.; Agbandje-McKenna, M.; Silverman, D. N.; Voth, G. A.; McKenna, R. Biochemistry 2007, 46, 2930.

(76) Williams, T. J.; Henkens, R. W. Biochemistry 1985, 24, 2459.

(77) Bertini, I.; Luchinat, C.; Monnanni, R.; Roelens, S.; Moratal, J. M. I. Am. Chem. Soc. 1987, 109, 7855.

(78) Krebs, J. F.; Rana, F.; Dluhy, R. A.; Fierke, C. A. Biochemistry 1993, 32, 4496.

(79) Liang, J. Y.; Lipscomb, W. N. Proc. Natl. Acad. Sci. U.S.A. 1990, $87,3675$.

(80) Fierke, C. A.; Calderone, T. L.; Krebs, J. F. Biochemistry 1991, 30, 11054.

(81) Alexander, R. S.; Nair, S. K.; Christianson, D. W. Biochemistry 1991, 30, 11064.

(82) Nair, S. K.; Calderone, T. L.; Christianson, D. W.; Fierke, C. A. I. Biol. Chem. 1991, 266, 17320.

(83) Nair, S. K.; Christianson, D. W. Biochemistry 1993, 32, 4506.

(84) Domsic, J. F.; McKenna, R. Biochim. Biophys. Acta 2010, 1804, 326.

(85) Supuran, C. T.; Conroy, C. W.; Maren, T. H. Proteins 1997, 27, 272.

(86) Briganti, F.; Mangani, S.; Scozzafava, A.; Vernaglione, G.; Supuran, C. T. I. Biol. Inorg. Chem. 1999, 4, 528.

(87) Guerri, A.; Briganti, F.; Scozzafava, A.; Supuran, C. T.; Mangani, S. Biochemistry 2000, 39, 12391.

(88) Pocker, Y.; Dickerson, D. G. Biochemistry 1968, 7, 1995.

(89) Pocker, Y.; Meany, J. E. Biochemistry 1967, 6, 239.

(90) Pocker, Y.; Stone, J. T. Biochemistry 1967, 6, 668.

(91) Verpoorte, J. A.; Mehta, S.; Edsall, J. T. L. Biol. Chem. 1967, 242, 4221.

(92) Kaiser, E. T.; Lo, K. W. L. Am. Chem. Soc. 1969, 91, 4912.

(93) Scozzafava, A.; Supuran, C. T. Bioorg. Med. Chem. 2003, 11, 2241.

(94) Temperini, C.; Innocenti, A.; Scozzafava, A.; Supuran, C. T. Bioorg. Med. Chem. Lett. 2006, 16, 4316.

(95) Scolnick, L. R.; Clements, A. M.; Liao, J.; Crenshaw, L.; Hellberg, M.; May, J.; Dean, T. R.; Christianson, D. W. $\underline{\text { L. Am. Chem. }}$ Soc. 1997, 119, 850 .

(96) Wildfeuer, A. Arzneimittelforschung 1970, 20, 824.

(97) Lius, V.; Sennerfeldt, P. Lakartidningen 1979, 76, 39.

(98) Goeth, H.; Wildfeuer, A. Arzneimittelforschung 1969, 19, 1298.

(99) Iyer, R.; Barrese, A. A., 3rd; Parakh, S.; Parker, C. N.; Tripp, B.

C. I. Biomol. Screen. 2006, 11, 782.

(100) Barrese, A. A., 3rd; Genis, C.; Fisher, S. Z.; Orwenyo, J. N.; Kumara, M. T; Dutta, S. K.; Phillips, E.; Kiddle, J. J.; Tu, C.; Silverman, D. N.; Govindasamy, L.; Agbandje-McKenna, M.; McKenna, R.; Tripp,

B. C. Biochemistry 2008, 47, 3174.
(101) Elleby, B.; Sjoblom, B.; Lindskog, S. Eur. J. Biochem. 1999, 262, 516

(102) Schulze Wischeler, J.; Innocenti, A.; Vullo, D.; Agrawal, A.; Cohen, S. M.; Heine, A.; Supuran, C. T.; Klebe, G. ChemMedChem 2010, 5, 1609.

(103) Temperini, C.; Scozzafava, A.; Supuran, C. T. Bioorg. Med. Chem. Lett. 2010, 20, 474.

(104) Liljas, A.; Håkansson, K.; Jonsson, B. H.; Xue, Y. Eur. I. Biochem. 1994, 219, 1.

(105) Mangani, S.; Håkansson, K. Eur. I. Biochem. 1992, $210,867$.

(106) Jönsson, B. M.; Håkansson, K.; Liljas, A. EEBS Lett. 1993, 322, 186.

(107) Nair, S. K.; Christianson, D. W. Eur. I. Biochem. 1993, 213, 507.

(108) Abbate, F.; Supuran, C. T.; Scozzafava, A.; Orioli, P.; Stubbs, M. T.; Klebe, G. I. Med. Chem. 2002, 45, 3583.

(109) Eriksson, A. E.; Jones, T. A.; Liljas, A. Proteins 1988, 4, 274.

(110) Keilin, D.; Mann, T. Biochem. I. 1940, 34, 1163.

(111) Winum, J.-Y.; Monter, J,-L.; Scozzafava, A.; Supuran, C. T. In Drug Design of Zinc-Enzvme Inhibitors: Functional, Structural, and Disease Applications; Supuran, C. T., Winum, J.-Y., Eds.; Wiley: Hoboken, NJ, 2009; p 39.

(112) King, R. W; Burgen, A. S. Biochim. Biophys. Acta 1970, 207, 278.

(113) Lindahl, M.; Vidgren, J.; Eriksson, E.; Habash, J.; Harrop, S.; Helliwell, J.; Liljas, A.; Lindeskog, M.;Walker, N. In Carbonic Anhydrase: From Biochemistry and Genetics to Physiology and Clinical Medicine; Botre, F., Gros, G., Storey, B. T., Eds.; VCH: Weinheim, Germany, 1991; p 111.

(114) Supuran, C. T.; Casini, A.; Scozzafava, A. In Carbonic Anhvdrase: Its inhibitors and Activators; Supuran, C. T., Scozzafava, A., Conway, J., Eds.; CRC Press: Boca Raton, FL, 2004; p 67.

(115) Beasley, Y. M.; Overell, B. G.; Petrow, V.; Stephenson, O. L. Pharm. Pharmacol. 1958, 10, 696.

(116) Supuran, C. T. In Carbonic Anhydrase and Modulation of Physiological and Pathologic Processes in the Organism; Puscas, I., Ed.; Helicon: Timisoara, 1994; p 29.

(117) Carta, F.; Pothen, B.; Maresca, A.; Tiwari, M.; Singh, V.; Supuran, C. T. Chem. Biol. Drug Des. 2009, 74, 196.

(118) Ilies, M. A.; Vullo, D.; Pastorek, J.; Scozzafava, A.; Ilies, M.; Caproiu, M. T.; Pastorekova, S.; Supuran, C. T. L. Med. Chem. 2003, 46, 2187.

(119) Scott, A. D.; Phillips, C.; Alex, A.; Flocco, M.; Bent, A.; Randall, A.; O’Brien, R.; Damian, L.; Jones, L. H. ChemMedChem 2009, 4, 1985.

(120) Mincione, F.; Starnotti, M.; Menabuoni, L.; Scozzafava, A.; Casini, A.; Supuran, C. T. Bioorg. Med. Chem. Lett. 2001, 11, 1787.

(121) Jain, A.; Whitesides, G. M.; Alexander, R. S.; Christianson, D. W. I. Med. Chem. 1994, 37, 2100.

(122) Gao, J.; Qiao, S.; Whitesides, G. M. I. Med. Chem. 1995, 38, 2292.

(123) Gao, J.; Cheng, X.; Chen, R.; Sigal, G. B.; Bruce, J. E.; Schwartz, B. L.; Hofstadler, S. A.; Anderson, G. A.; Smith, R. D.; Whitesides, G. M. I. Med. Chem. 1996, 39, 1949.

(124) Roy, B. C.; Banerjee, A. L.; Swanson, M.; Jia, X. G.; Haldar, M. K.; Mallik, S.; Srivastava, D. K. I. Am. Chem. Soc. 2004, 126, 13206.

(125) Jude, K. M.; Banerjee, A. L.; Haldar, M. K.; Manokaran, S.; Roy, B.; Mallik, S.; Srivastava, D. K.; Christianson, D. W. L. Am. Chem. Soc. 2006, 128, 3011.

(126) Doyon, J. B.; Jain, A. Org. Lett. 1999, 1, 183.

(127) Cappalonga Bunn, A. M.; Alexander, R. S.; Christianson, D. W. L. Am. Chem. Soc. 1994, 116, 5063.

(128) Jain, A.; Huang, S. G.; Whitesides, G. M. I. Am. Chem. Soc. 1994, 116, 5057.

(129) Boriack, P. A.; Christianson, D. W.; Kingery-Wood, J.; Whitesides, G. M. I. Med. Chem. 1995, 38, 2286.

(130) Banerjee, A. L.; Swanson, M.; Roy, B. C.; Jia, X.; Haldar, M. K.; Mallik, S.; Srivastava, D. K. I. Am. Chem. Soc. 2004, 126, 10875.

(131) Chin, D. N.; Whitesides, G. M. I. Am. Chem. Soc. 1995, 117, 6153. 
(132) Chin, D. N.; Lau, A. Y.; Whitesides, G. M. I. Org. Chem. 1998, 63, 938.

(133) Casini, A.; Scozzafava, A.; Mincione, F.; Menabuoni, L.; Ilies, M. A.; Supuran, C. T I. Med. Chem. 2000, 43, 4884.

(134) Casini, A.; Scozzafava, A.; Mincione, F.; Menabuoni, L.; Supuran, C. T. I. Enzyme Inhib. Med. Chem 2002, 17, 333.

(135) Cecchi, A.; Winum, J.-Y.; Innocenti, A.; Vullo, D.; Montero, J.L.; Scozzafava, A.; Supuran, C. T. Bioorg. Med. Chem. Lett. 2004, 14, 5775 .

(136) Innocenti, A.; Casini, A.; Alcaro, M. C.; Papini, A. M.; Scozzafava, A.; Supuran, C. T. I. Med. Chem. 2004, 47, 5224.

(137) Pacchiano, F.; Aggarwal, M.; Avvaru, B. S.; Robbins, A. H.; Scozzafava, A.; McKenna, R.; Supuran, C. T. Chem. Commun. 2010, 46, 8371.

(138) Supuran, C. T.; Scozzafava, A.; Jurca, B. C.; Ilies, M. A. Eur. I. Med. Chem. 1998, 33, 83.

(139) Di Fiore, A.; De Simone, G.; Menchise, V.; Pedone, C.; Casini, A.; Scozzafava, A.; Supuran, C. T. Bioorg. Med. Chem. Lett. 2005, 15, 1937.

(140) Alterio, V.; Vitale, R. M.; Monti, S. M.; Pedone, C.; Scozzafava, A.; Cecchi, A.; De Simone, G.; Supuran, C. T. L.Am. Chem. Soc. 2006, $128,8329$.

(141) Ciani, L.; Cecchi, A.; Temperini, C.; Supuran, C. T.; Ristori, S. I. Phvs. Chem. B 2009, 113, 13998.

(142) Pastorekova, S.; Casini, A.; Scozzafava, A.; Vullo, D.; Pastorek, J.; Supuran, C. T. Bioorg. Med. Chem. Lett. 2004, 14, 869.

(143) Supuran, C. T.; Scozzafava, A.; Ilies, M. A.; Briganti, F. I. Enzvme Inhib. 2000, 15, 381.

(144) Supuran, C. T.; Scozzafava, A. I. Enzyme Inhib. Med. Chem. 2004, 19, 269.

(145) Supuran, C. T.; Manole, G.; Dinculescu, A.; Schiketanz, A.; Gheorghiu, M. D.; Puscas, I.; Balaban, A. T. L. Pharm. Sci. 1992, 81, 716.

(146) Supuran, C. T.; Scozzafava, A.; Ilies, M. A.; Iorga, B.; Cristea, T.; Briganti, F.; Chiraleu, F.; Banciu, M. D. Eur. I. Med. Chem. 1998, 33, 577.

(147) Scozzafava, A.; Briganti, F.; Ilies, M. A.; Supuran, C. T. L. Med. Chem. 2000, 43, 292.

(148) Casey, J. R.; Morgan, P. E.; Vullo, D.; Scozzafava, A.; Mastrolorenzo, A.; Supuran, C. T. L. Med. Chem. 2004, 47, 2337.

(149) Balaban, A. T.; Dinculescu, A. Rev. Roum. Chim. 1980, 28, 1505

(150) Menchise, V.; De Simone, G.; Alterio, V.; Di Fiore, A.; Pedone, C.; Scozzafava, A.; Supuran, C. T. I. Med. Chem. 2005, 48, 5721.

(151) Svastová, E.; Hulíková, A.; Rafajová, M.; Zat'ovicová, M.; Gibadulinová, A.; Casini, A.; Cecchi, A.; Scozzafava, A.; Supuran, C. T.; Pastorek, J.; Pastoreková, S. FEBS Lett. 2004, 577, 439.

(152) Srivastava, D. K.; Jude, K. M.; Banerjee, A. L.; Haldar, M.; Manokaran, S.; Kooren, J.; Mallik, S.; Christianson, D. W. I. Am. Chem. Soc. 2007, 129, 5528 .

(153) Alterio, V.; De Simone, G.; Monti, S. M.; Scozzafava, A.; Supuran, C. T. Bioorg. Med. Chem. Lett. 2007, 17, 4201.

(154) Abbate, F.; Casini, A.; Owa, T.; Scozzafava, A.; Supuran, C. T. Bioorg. Med. Chem. Lett. 2004, 14, 217.

(155) Casini, A.; Abbate, F.; Scozzafava, A.; Supuran, C. T. Bioorg. Med. Chem. Lett. 2003, 13, 2759.

(156) Mincione, F.; Menabuoni, L.; Supuran, C. T. In Carbonic Anhydrase, Its Inhibitors and Activators; Supuran, C. T., Scozzafava, A., Conway, J., Eds.; CRC Press: Boca Raton, FL, 2004; p 243.

(157) Tawil, R.; McDermott, M. P.; Brown, R., Jr; Shapiro, B. C.; Ptacek, L. J.; McManis, P. G.; Dalakas, M. C.; Spector, S. A.; Mendell, J. R.; Hahn, A. F.; Griggs, R. C. Ann. Neurol. 2000, 47, 46.

(158) Pastoreková, S.; Závadová, Z.; Kostál, M.; Babusíková, O.; Závada, I. Virology 1992, 187, 620.

(159) Türeci, O.; Sahin, U.; Vollmar, E.; Siemer, S.; Göttert, E.; Seitz, G.; Parkkila, A. K.; Shah, G. N.; Grubb, J. H.; Pfreundschuh, M.; Sly, W. S. Proc. Natl. Acad. Sci. U.S.A. 1998, 95, 7608.
(160) Ivanov, S. V.; Kuzmin, I.; Wei, M. H.; Pack, S.; Geil, L.; Johnson, B. E.; Stanbridge, E. J.; Lerman, M. I. Proc. Natl. Acad. Sci. U.S.A. 1998, 95, 12596.

(161) Pastoreková, S.; Pastorek, J. In Carbonic Anhvdrase: Its Inhibitors and Activators; Supuran, C. T., Scozzafava, A., Conway, J., Eds.; CRC Press: Boca Raton, FL, 2004; p 255.

(162) Aaron, J. A.; Chambers, J. M.; Jude, K. M.; Di Costanzo, L.; Dmochowski, I. J.; Christianson, D. W. I. Am. Chem. Soc. 2008, 130, 6942.

(163) Cecchi, A.; Hulikova, A.; Pastorek, J.; Pastoreková, S.; Scozzafava, A.; Winum, J.-Y.; Montero, J.-L.; Supuran, C. T. I. Med. Chem. 2005, 48, 4834.

(164) Elbaum, D.; Nair, S. K.; Patchan, M. W.; Thompson, R. B.; Christianson, D. W. L. Am. Chem. Soc. 1996, 118, 8381.

(165) Marsh, D.; Horváth, L. I. In Advanced EPR. Applications in Biology and Biochemistry; Hoff, A. J., Ed.; Elsevier: Amsterdam, 1989; p 707.

(166) Schiemann, O.; Prisner, T. F. Q. Rev. Biophvs. 2007, 40, 1.

(167) Taylor, J. S.; Mushak, P.; Coleman, J. E. Proc. Natl. Acad. Sci. U.S.A. 1970, 67, 1410 .

(168) Mushak, P.; Coleman, J. E. I. Biol. Chem. 1972, 247, 373.

(169) Chignell, C. F.; Starkweather, D. K.; Erlich, R. H. I. Med. Chem. 1972, 15, 876.

(170) Erlich, R. H.; Starkweather, D. K.; Chignell, C. F. Mol. Pharmacol. 1973, 9, 61 .

(171) Hower, J. F.; Henkens, R. W.; Chesnut, D. B. I. Am. Chem. Soc. 1971, 93, 6665 .

(172) Cecchi, A.; Supuran, C. T. Curr. Pharm. Des 2008, 14, 699.

(173) Wee, V. T.; Feldmann, R. J.; Tanis, R. J.; Chignell, C. F. Mol. Pharmacol. 1976, 12, 832.

(174) Sotgiu, A.; Mader, K.; Placidi, G.; Colacicchi, S.; Ursini, C. L.; Alecci, M. Phvs. Med. Biol. 1998, 43, 1921.

(175) Cecchi, A.; Ciani, L.; Winum, J.-Y.; Montero, J.-L.; Scozzafava, A.; Ristori, S.; Supuran, C. T. Bioorg. Med. Chem. Lett. 2008, 18, 3475.

(176) Raftery, D. Annu. Rep. NMR Spectrosc. 2006, 57, 205.

(177) Cherubini, A.; Bifone, A. Prog. Nucl. Magn. Reson. Spectrosc. 2003, 42, 1.

(178) Fain, S. B.; Korosec, F. R.; Holmes, J. H.; O'Halloran, R.; Sorkness, R. L.; Grist, T. M. I. Magn. Reson. Imaging 2007, 25, 910.

(179) Hill, P. A.; Wei, Q.; Eckenhoff, R. G.; Dmochowski, I. J. I. Am. Chem. Soc. 2007, 129, 9262.

(180) Lowery, T. J.; Garcia, S.; Chavez, L.; Ruiz, E. J.; Wu, T.; Brotin, T.; Dutasta, J. P.; King, D. S.; Schultz, P. G.; Pines, A.; Wemmer, D. E. ChemBioChem 2006, 7, 65.

(181) Schröder, L.; Lowery, T. J.; Hilty, C.; Wemmer, D. E.; Pines, A Science 2006, 314, 446.

(182) Chambers, J. M.; Hill, P. A.; Aaron, J. A.; Han, Z.; Christianson, D. W.; Kuzma, N. N.; Dmochowski, I. J. I. Am. Chem. Soc. 2009, 131, 563.

(183) Bodor, N.; Buchwald, P. In Burger's Medicinal Chemistry Drug Discovery and Development; Abraham, D. J., Ed; John Wiley and Sons, New York, 2003, p 533.

(184) Denny, W. A. Cancer Invest. 2004, 22, 604.

(185) De Simone, G.; Vitale, R. M.; Di Fiore, A.; Pedone, C.; Scozzafava, A.; Montero, J.-L.; Winum, J.-Y.; Supuran, C. T. I. Med. Chem. 2006, 49, 5544.

(186) D’Ambrosio, K.; Vitale, R. M.; Dogné, J. M.; Masereel, B.; Innocenti, A.; Scozzafava, A.; De Simone, G.; Supuran, C. T. I. Med. Chem. 2008, 51, 3230.

(187) Saczewski, F.; Sławinski, J.; Kornicka, A.; Brzozowski, Z.; Pomarnacka, E.; Innocenti, A.; Scozzafava, A.; Supuran, C. T. $\underline{\text { Bioorg. }}$ Med. Chem. Lett. 2006, 16, 4846.

(188) Davenport, H. W. I. Biol. Chem. 1945, 158, 567.

(189) Miller, W. H.; Dessert, A. M.; Roblin, R. O., Jr. I. Am. Chem. Soc. 1950, 72, 4893.

(190) Roblin, R. O., Jr.; Clapp, J. W. I. Am. Chem. Soc. 1950, 72, 4890.

(191) Barnish, I. T.; Cross, P. E.; Dickinson, R. P.; Parry, M. J.; Randall, M. J. I.Med. Chem. 1981, 24, 959. 
(192) Maren, T. H. I. Glaucoma 1995, 4, 492.

(193) Vidgren, J.; Liljas, A.; Walker, N. P. C Int. I. Biol. Macromol. 1990, 12, 342

(194) Abbate, F.; Casini, A.; Scozzafava, A.; Supuran, C. T. $\underline{\text {. Enzvme }}$ Inhib. Med. Chem. 2003, 18, 303.

(195) Abbate, F.; Casini, A.; Scozzafava, A.; Supuran, C. T. Bioorg. Med. Chem. Lett. 2004, 14, 2357.

(196) Boriack-Sjodin, P. A.; Zeitlin, S.; Chen, H. H.; Crenshaw, L.; Gross, S.; Dantanarayana, A.; Delgado, P.; May, J. A.; Dean, T.; Christianson, D. W. Protein Sci. 1998, 7, 2483.

(197) Menchise, V.; De Simone, G.; Di Fiore, A.; Scozzafava, A.; Supuran, C. T. Bioorg. Med. Chem. Lett. 2006, 16, 6204.

(198) Vaughan, J. R.; Eichler, J. A.; Anderson, G. W. I. Org. Chem. 1956, 21, 700 .

(199) Young, R. W.; Wood, K. H.; Vaughan, J. R.; Anderson, G. W. I. Am. Chem. Soc. 1956, 78, 4649.

(200) Supuran, C. T.; Ilies, M. A.; Scozzafava, A. Eur. I. Med. Chem. 1998, 33, 739.

(201) Casini, A.; Scozzafava, A.; Mincione, F.; Menabuoni, L.; Starnotti, M.; Supurana, C. T. Bioorg. Med. Chem. Lett. 2003, 13, 2867.

(202) Scozzafava, A.; Menabuoni, L.; Mincione, F.; Briganti, F.; Mincione, G.; Supuran, C. T. I. Med. Chem. 2000, 43, 4542.

(203) Chow, K.; Lai, R.; Holmes, J. M.; Wijono, M.; Wheeler, L. A.; Garst, M. E. Eur. I. Med. Chem. 1996, 31, 175.

(204) Supuran, C. T.; Scozzafava, A. Expert Opin. Ther. Pat. 2000, 10, 575 .

(205) Supuran, C. T.; Scozzafava, A. Expert. Opin. Ther. Pat. 2002, 12, 217.

(206) Maren, T. H. In Orphan Drugs; Karch, F. E., Ed.; Dekker: New York, 1982; p 89.

(207) de Leval, X.; Ilies, M.; Casini, A.; Dogné, J. M.; Scozzafava, A.; Masini, E.; Mincione, F.; Starnotti, M.; Supuran, C. T. $\underline{\text { I. Med. Chem. }}$ 2004, 47, 2796.

(208) Kim, C. Y.; Whittington, D. A.; Chang, J. S.; Liao, J.; May, J. A.; Christianson, D. W. I. Med. Chem. 2002, 45, 888.

(209) Smith, G. M.; Alexander, R. S.; Christianson, D. W.; McKeever, B. M.; Ponticello, G. S.; Springer, J. P.; Randall, W. C.; Baldwin, J. J.; Habecker, C. N. Protein Sci. 1994, 3, 118.

(210) Ponticello, G. S.; Freedman, M. B.; Habecker, C. N.; Lyle, P. A.; Schwam, H.; Varga, S. L.; Christy, M. E.; Randall, W. C.; Baldwin, J. J. I. Med. Chem. 1987, 30, 591.

(211) Steele, R. M.; Benedini, F.; Biondi, S.; Borghi, V.; Carzaniga, L.; Impagnatiello, F.; Miglietta, D.; Chong, W. K.; Rajapakse, R.; Cecchi, A.; Temperini, C.; Supuran, C. T. Bioorg. Med. Chem. Lett. 2009, 19, 6565.

(212) Kotikoski, H.; Alajuuma, P.; Moilanen, E.; Salmenperä, P.; Oksala, O.; Laippala, P.; Vapaatalo, H. I. Ocul. Pharmacol. Ther. 2002, $18,11$.

(213) Galassi, F.; Renieri, G.; Sodi, A.; Ucci, F.; Vannozzi, L.; Masini, E. L. Ophthalmol. 2004, 88, 757.

(214) Nathanson, J. A. I. Pharmacol. Exp. Ther. 1992, 260, 956.

(215) Kotikoski, H.; Vapaatalo, H.; Oksala, O. Curr. Eve Res. 2003, 26, 119.

(216) Becquet, F.; Courtois, Y.; Goureau, O. Surv. Ophthalmol. 1997, $42,71$.

(217) D’Ambrosio, K.; Masereel, B.; Thiry, A.; Scozzafava, A.; Supuran, C. T; De Simone, G. ChemMedChem 2008, 3, 473.

(218) Gruneberg, S.; Stubbs, M. T.; Klebe, G. I. Med. Chem. 2002, $45,3588$.

(219) Güzel, Ö.; Temperini, C.; Innocenti, A.; Scozzafava, A.; Salman, A.; Supuran, C. T. Bioorg. Med. Chem. Lett. 2008, 18, 152.

(220) Crocetti, L.; Maresca, A.; Temperini, C.; Hall, R. A.; Scozzafava, A.; Mühlschlegel, F. A.; Supuran, C. T. Bioorg. Med. Chem. Lett. 2009, 19, 1371.

(221) Gitto, R.; Agnello, S.; Ferro, S.; De Luca, L.; Vullo, D.; Brynda, J.; Mader, P.; Supuran, C. T.; Chimirri, A. I. Med.Chem. 2010, 53, 2401.
(222) Gitto, R.; Ferro, S.; Agnello, S.; De Luca, L.; De Sarro, G.; Russo, E.; Vullo, D.; Supuran, C. T.; Chimirri, A. Bioorg. Med. Chem. 2009, 17, 3659.

(223) Güzel, O.; Innocenti, A.; Scozzafava, A.; Salman, A.; Parkkila, S.; Hilvo, M.; Supuran, C. T. Bioorg. Med. Chem. 2008, 16, 9113.

(224) Luz, C.; Netto, M. C.; Rocha, L. F. Mvcopathologia 2007, 164, 39.

(225) Dalvie, D.; Smith, E.; Deese, A.; Bowlin, S. Druq Metab. Dispos. 2006, 34, 709.

(226) Martin, R. J.; Robertson, A. P.; Bjorn, H. Parasitology 1997, 114, S111.

(227) Smith, R. E.; Ashiya, M. Nat. Rev. Drug Discovery 2007, 6, 597.

(228) Salvetti, A.; Ghiadoni, L. L. Am. Soc. Nephrol. 2006, 17, S25.

(229) Padilla, M. C.; Armas-Hernández, M. J.; Hernández, R. H.; Israili, Z. H.; Valasco, M. Am. I. Ther. 2007, 14, 154.

(230) Nishioku, T.; Takata, F.; Yamauchi, A.; Sumi, N.; Yamamoto, I.; Fujino, A.; Naito, M.; Tsuruo, T.; Shuto, H.; Kataoka, Y. I. Pharmacol. Sci. 2007, 103, 323.

(231) Dell'Omo, G.; Penno, G.; Del Prato, S.; Pedrinelli, R. I. Cardiovasc. Pharmacol. Ther. 2005, 10, 265.

(232) Black, H. R.; Davis, B.; Barzilay, J.; Nwachuku, C.; Baimbridge, C.; Marginean, H.; Wright, J. T., Jr; Basile, J.; Wong, N. D.; Whelton, P.; Dart, R. A.; Thadani, U. Diabetes Care 2008, 31, 353.

(233) Struijker-Boudier, H. A. Am. I. Hvpertension 2007, 20, $15 \mathrm{~S}$.

(234) Patel, A.; MacMahon, S.; Chalmers, J.; Neal, B.; Woodward, M.; Billot, L.; Harrap, S.; Poulter, N.; Marre, M.; Cooper, M.; Glasziou, P.; Grobbee, D. E.; Hamet, P.; Heller, S.; Liu, L. S.; Mancia, G.; Mogensen, C. E.; Pan, C. Y.; Rodgers, A.; Williams, B. Lancet 2007, 370,829 .

(235) Temperini, C.; Cecchi, A.; Scozzafava, A.; Supuran, C. T. Bioorg. Med. Chem. Lett. 2008, 18, 2567.

(236) Temperini, C.; Cecchi, A.; Scozzafava, A.; Supuran, C. T. Org. Biomol. Chem. 2008, 6, 2499.

(237) Temperini, C.; Cecchi, A.; Scozzafava, A.; Supuran, C. T. Bioorg. Med. Chem. 2009, 17, 1214.

(238) Vullo, D.; Innocenti, A.; Supuran, C. T. In Drug Design of ZincEnzyme Inhibitors: Functional, Structural, and Disease Applications; Supuran, C. T., Winum, J.-Y., Eds.; Wiley: Hoboken, NJ, 2009; p 155.

(239) Temperini, C.; Cecchi, A.; Scozzafava, A.; Supuran, C. T. I. Med. Chem. 2009, 52, 322.

(240) Maren, T. H.; Conroy, C. W. I. Biol. Chem. 1993, 268, 26233.

(241) Håkansson, K.; Liljas, A. FEBS Lett. 1994, 350, 319.

(242) De Simone, G.; Di Fiore, A.; Menchise, V.; Pedone, C.; Antel, J.; Casini, A.; Scozzafava, A.; Wurl, M.; Supuran, C. T. Bioorg. Med. Chem. Lett. 2005, 15, 2315.

(243) Temperini, C.; Cecchi, A.; Boyle, N. A.; Scozzafava, A.; Cabeza, J. E.; Wentworth, P.; Blackburn, G. M.; Supuran, C. T. Bioorg. Med. Chem. Lett. 2008, 18, 999.

(244) Fisher, S. Z.; Govindasamy, L.; Boyle, N.; Agbandje-McKenna, M.; Silverman, D. N.; Blackburn, G. M.; McKenna, R. Acta Crystallogr. Sect. F. 2006, 62, 618.

(245) Lo, Y. S.; Nolan, J. C.; Maren, T. H.; Welstead, W. J., Jr; Gripshover, D. F.; Shamblee, D. A. I. Med. Chem. 1992, 35, 4790.

(246) Maryanoff, B. E.; Nortey, S. O.; Gardocki, J. F.; Shank, R. P.; Dodgson, S. P. I. Med. Chem. 1987, 30, 880.

(247) Briganti, F.; Pierattelli, R; Scozzafava, A.; Supuran, C. T. Eur. I. Med. Chem. 1996, 31, 1001.

(248) Thiry, A.; Dogné, J. M.; Supuran, C. T.; Masereel, B. Curr. Top. Med. Chem. 2007, 7, 855 .

(249) Winum, J.-Y.; Vullo, D.; Casini, A.; Montero, J.-L.; Scozzafava, A.; Supuran, C. T. I. Med. Chem. 2003, 46, 2197.

(250) Winum, J.-Y.; Vullo, D.; Casini, A.; Montero, J.-L.; Scozzafava, A.; Supuran, C. T. I. Med. Chem. 2003, 46, 5471.

(251) Winum, J.-Y.; Pastorekova, S.; Jakubickova, L.; Montero, J.-L.; Scozzafava, A.; Pastorek, J.; Vullo, D.; Innocenti, A.; Supuran, C. T. Bioorg. Med. Chem. Lett. 2005, 15, 579.

(252) Vitale, R. M.; Alterio, V.; Innocenti, A.; Winum, J.-Y.; Monti, S.

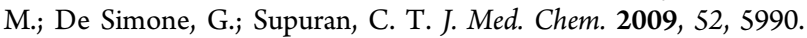


(253) Reed, M. J.; Purohit, A.; Newman, S. P.; Potter, B. V. L Endocr. Rev. 2005, 26, 171.

(254) Stanway, S. J.; Purohit, A.; Woo, L. W. L.; Sufi, S.; Vigushin, D.; Ward, R.; Wilson, R.; Stanczyk, F. Z.; Dobbs, N.; Kulinkaya, E.; Elliott, M.; Potter, B. V. L; Reed, M. J.; Coombs, R. C. Clin. Cancer Res. 2006, 12, 1585 .

(255) Ireson, C. R.; Chander, S. K.; Purohit, A.; Parish, D. A.; Woo, L. W. L.; Potter, B. V. L; Reed, M. J. Br. I. Cancer 2004, 91, 1399.

(256) Abbate, F.; Winum, J. Y.; Potter, B. V. L; Casini, A.; Montero, J. L.; Scozzafava, A.; Supuran, C. T. Bioorg. Med. Chem. Lett. 2004, 14, 231.

(257) Lloyd, M. D.; Pederick, R. L.; Natesh, R.; Woo, L. W. L.; Purohit, A.; Reed, M. J.; Acharya, K. R.; Potter, B.V. L. Biochem. I. 2005, 385, 715 .

(258) Lloyd, M. D.; Thiyagarajan, N.; Ho, Y. T.; Woo, L. W. L.; Sutcliffe, O. B.; Purohit, A.; Reed, M. J.; Acharya, K. R.; Potter, B. V. L Biochemistry 2005, 44, 6858.

(259) Leese, M. P.; Leblond, B.; Smith, A.; Newman, S. P.; Di Fiore, A.; De Simone, G.; Supuran, C. T.; Purohit, A.; Reed, M. J.; Potter, B. V. I. Med. Chem. 2006, 49, 7683.

(260) Leese, M. P.; Jourdan, F. L.; Gaukroger, K.; Mahon, M. F.; Newman, S. P.; Foster, P. A.; Stengel, C.; Regis-Lydi, S.; Ferrandis, E.; Di Fiore, A.; De Simone, G.; Supuran, C. T.; Purohit, A.; Reed, M. J.; Potter, B. V. I. Med. Chem. 2008, 51, 1295.

(261) Woo, L.W. L.; Fischer, D. S.; Sharland, C. M.; Trusselle, M.; Foster, P. A.; Chander, S. K.; Di Fiore, A.; Supuran, C. T.; De Simone, G.; Purohit, A.; Reed, M. J.; Potter, B.V. L. Mol. Cancer Ther. 2008, 7, 2435.

(262) Woo, L. W.; Jackson, T.; Putey, A.; Cozier, G.; Leonard, P.; Acharya, K. R.; Chander, S. K.; Purohit, A.; Reed, M. J.; Potter, B. V. I. Med. Chem. 2010, 53, 2155.

(263) Cozier, G. E.; Leese, M. P.; Lloyd, M. D.; Baker, M. D.; Thiyagarajan, N.; Acharya, K. R.; Potter, B. V. Biochemistry 2010, 49, 3464.

(264) Howarth, N. M.; Purohit, A.; Reed, M. J.; Potter, B. V. L I. Med. Chem. 1994, 37, 219.

(265) Elger, W.; Schwarz, S.; Hedden, A. M.; Reddersen, G.; Schneider, B. I. Steroid Biochem. Mol. Biol. 1995, 55, 395.

(266) Purohit, A.; Woo, L. W. L.; Potter, B. V. L; Reed, M. J. Cancer Res. 2000, 60, 3394.

(267) Foster, P. A.; Newman, S. P.; Chander, S. K.; Stengel, C.; Jhalli, R.; Woo, L. L.; Potter, B. V.; Reed, M. J.; Purohit, A. Clin. Cancer Res. 2006, 12, 5543.

(268) Foster, P. A.; Chander, S. K.; Parsons, M. F.; Newman, S. P.; Woo, L. W.; Potter, B. V.; Reed, M. J.; Purohit, A. Breast Cancer Res. Treat. 2008, 111, 129.

(269) Fischer, D. S.; Woo, L. W.; Mahon, M. F.; Purohit, A.; Reed, M. J.; Potter, B. V. Bioorg. Med. Chem. 2003, 11, 1685.

(270) Fischer, D. S.; Chander, S. K.; Woo, L. W.; Fenton, J. C.; Purohit, A.; Reed, M. J.; Potter, B. V. L. Steroid. Biochem. Mol. Biol. 2003, 84, 343.

(271) Woo, L.W. L.; Sutcliffe, O. B.; Bubert, C.; Grasso, A.; Chander, S. K.; Purohit, A.; Reed, M. J.; Potter, B. V. L J. Med. Chem. 2003, 46, 3193.

(272) Wilkinson, B. L.; Bornaghi, L. F.; Houston, T. A.; Innocenti, A.; Supuran, C. T.; Poulsen, S. A. L.Med. Chem. 2006, 49, 6539.

(273) Wilkinson, B. L.; Bornaghi, L. F.; Houston, T. A.; Innocenti, A.; Vullo, D.; Supuran, C. T.; Poulsen, S. A. Bioorg. Med. Chem. Lett. 2007, 17, 987.

(274) Wilkinson, B. L.; Bornaghi, L. F.; Houston, T. A.; Innocenti, A.; Vullo, D.; Supuran, C. T.; Poulsen, S. A. I. Med. Chem. 2007, 50, 1651.

(275) Winum, J.-Y.; Poulsen, S.-A.; Supuran, C. T. Med. Res. Rev. 2009, 29, 419.

(276) Winum, J.-Y.; Casini, A.; Minzione, F.; Starnotti, M.; Montero, J.-L.; Scozzafava, A.; Supuran, C. T. Bioorg. Med. Chem. Lett. 2004, 14, 225.

(277) Colinas, P. A.; Bravo, R. D.; Vullo, D.; Scozzafava, A.; Supuran, C. T. Bioorg. Med. Chem. Lett. 2007, 17, 5086.
(278) Di Fiore, A.; Scozzafava, A.; Winum, J.-Y.; Montero, J.-L.; Pedone, C.; Supuran, C. T.; De Simone, G. Bioorg. Med. Chem. Lett. 2007, 17, 1726.

(279) Shank, R. P.; Gardocki, J. F.; Vaught, J. L.; Davis, C. B.; Schupsky, J. J.; Raffa, R. B.; Dodgson, S. J.; Nortey, S. O.; Maryanoff, B. E. Epilepsia 1994, 35, 450.

(280) Edmonds, H. L., Jr; Jiang, Y. D.; Zhang, P. Y.; Shank, R. P. Life Sci. 1996, 59, PL127.

(281) Stringer, J. L. Epilepsv Res. 2000, 40, 147.

(282) Sabers, A.; Gram, L. Drugs 2000, 60, 23.

(283) Bourgeois, B. F. L. Child Neurol. 2000, 15, S27.

(284) Bialer, M.; Johannessen, S. I.; Kupferberg, H. J.; Levy, R. H.; Loiseau, P.; Perucca, E. Epilepsy Res. 2001, 43, 11.

(285) Gordon, A.; Price, L. H. Am. I. Psvchiatry 1999, 156, 968.

(286) Casini, A.; Antel, J.; Abbate, F.; Scozzafava, A.; David, S.; Waldeck, H.; Schafer, S.; Supuran, C. T. Bioorg. Med. Chem. Lett. 2003, 13, 841 .

(287) Vitale, R. M.; Pedone, C.; Amodeo, P.; Antel, J.; Wurl, M.; Scozzafava, A.; Supuran, C. T.; De Simone, G. Bioorg. Med. Chem. 2007, 15, 4152.

(288) Winum, J.-Y.; Temperini, C.; El Cheikh, K.; Innocenti, A.; Vullo, D.; Ciattini, S.; Montero, J.-L.; Scozzafava, A.; Supuran, C. T. I. Med. Chem. 2006, 49, 7024.

(289) Maryanoff, B. E.; Costanzo, M. J.; Nortey, S. O.; Greco, M. N.; Shank, R. P.; Schupsky, J. J.; Ortegon, M. E.; Vaught, J. L. I.Med. Chem. 1998, 41, 1315.

(290) Recacha, R.; Costanzo, M. J.; Maryanoff, B. E.; Chattopadhyay, D. Biochem. I. 2002, 361, 437.

(291) Lopez, M.; Paul, B.; Hofmann, A.; Morizzi, J.; Wu, Q. K.; Charman, S. A.; Innocenti, A.; Vullo, D.; Supuran, C. T.; Poulsen, S. A. I. Med. Chem. 2009, 52, 6421.

(292) Lopez, M.; Drillaud, N.; Bornaghi, L. F.; Poulsen, S. A. I. Org. Chem. 2009, 74, 2811.

(293) Simonsson, I.; Jonsson, B. H.; Lindskog, S. Biochem. Biophys. Res. Commun. 1982, 108, 1406.

(294) Tibell, L.; Forsman, C.; Simonsson, I.; Lindskog, S. Biochim. Biophvs. Acta 1985, 829, 202.

(295) Nair, S. K.; Ludwig, P. A.; Christianson, D. W. I. Am. Chem. Soc. 1994, 116, 3659.

(296) Davis, R. A.; Hofmann, A.; Osman, A.; Hall, R. A.; Mühlschlegel, F. A.; Vullo, D.; Innocenti, A.; Supuran, C. T.; Poulsen, S. A. L. Med. Chem. 2011, 54, 1682.

(297) Carta, F.; Temperini, C.; Innocenti, A.; Scozzafava, A.; Kaila, K.; Supuran, C. T. I. Med. Chem. 2010, 53, 5511.

(298) Maresca, A.; Temperini, C.; Vu, H.; Pham, N. B.; Poulsen, S. A.; Scozzafava, A.; Quinn, R. J.; Supuran, C. T. I. Am. Chem. Soc. 2009, $131,3057$.

(299) Maresca, A.; Temperini, C.; Pochet, L.; Masereel, B.; Scozzafava, A.; Supuran, C. T. I.Med. Chem. 2010, 53, 335.

(300) Temperini, C.; Innocenti, A.; Scozzafava, A.; Parkkila, S.; Supuran, C. T. I. Med. Chem. 2010, 53, 850.

(301) Görlach, A.; Kietzmann, T. Methods Enzvmol. 2007, 435, 421.

(302) BelAiba, R. S.; Djordjevic, T.; Petry, A.; Diemer, K.; Bonello, S.; Banfi, B.; Hess, J.; Pogrebniak, A.; Bickel, C.; Görlach, A. Free Radic. Biol. Med. 2007, 42, 446.

(303) Bell, E. L.; Klimova, T. A.; Eisenbart, J.; Moraes, C. T.; Murphy, M. P.; Budinger, G. R.; Chandel, N. S. I. Cell Biol. 2007, 177, 1029.

(304) Bonello, S.; Zähringer, C.; BelAiba, R. S.; Djordjevic, T.; Hess, J.; Michiels, C.; Kietzmann, T.; Görlach, A. Arterioscler. Thromb. Vasc. Biol. 2007, 27, 755.

(305) Gülçin, I. Toxicology 2006, 217, 213.

(306) Gülçin, I. Amino Acids 2007, 32, 431.

(307) Gülçin, I.; Bursal, E.; Sehitoğlu, M. H.; Bilsel, M.; Gören, A. C. Food Chem. Toxicol. 2010, 48, 2227.

(308) The Flavonoids; Mabry, H., Harborne, J. B., Mabry, T. J., Eds.; Chapman and Hall: London, 1975.

(309) Köksal, E.; Gülçin, I.; Beyza, S.; Sarikaya, O.; Bursal, E. I. Enzvme Inhib. Med. Chem. 2009, 24, 395. 
(310) Huseini, H. F.; Larijani, B.; Heshmat, R.; Fakhrzadeh, H.; Radjabipour, B.; Toliat, T.; Raza, M. Phytother. Res. 2006, 20, 1036.

(311) Ak, T.; Gülçin, I. Chem. Biol. Interact. 2008, 174, 27.

(312) Huang, M. T.; Newmark, H. L.; Frenkel, K. I. Cell Biochem. 1997, 27, 26.

(313) Merz, K. M. I. Mol. Biol. 1990, 214, 799.

(314) Khalifah, R. G.; Rogers, J. I. FASEB J 1988, 2, A1118.

(315) Innocenti, A.; Vullo, D.; Scozzafava, A.; Supuran, C. T. Bioorg. Med. Chem. Lett. 2008, 18, 1583.

(316) Innocenti, A.; Vullo, D.; Scozzafava, A.; Supuran, C. T. Bioorg. Med. Chem. 2008, 16, 7424.

(317) Innocenti, A.; Gülçin, I.; Scozzafava, A.; Supuran, C. T. Bioorg. Med. Chem. Lett. 2010, 20, 5050.

(318) Innocenti, A.; Beyza Oztürk Sarikaya, S.; Gülçin, I.; Supuran, C. T. Bioorg. Med. Chem. 2010, 18, 2159.

(319) Sarikaya, S. B.; Gülçin, I.; Supuran, C. T. Chem. Biol. Drug Des. 2010, 75, 515.

(320) Briganti, F.; Mangani, S.; Orioli, P.; Scozzafava, A.; Vernaglione, G.; Supuran, C. T. Biochemistry 1997, 36, 10384.

(321) Clare, B. W.; Supuran, C. T. I. Pharm. Sci. 1994, 83, 768.

(322) Ilies, M.; Banciu, M. D.; Ilies, M. A.; Scozzafava, A.; Caproiu, M. T.; Supuran, C. T. I. Med. Chem. 2002, 45, 504.

(323) Ilies, M.; Scozzafava, A.; Supuran, C. T. In Carbonic Anhydrase: Its Inhibitors and Activators; Supuran, C. T., Scozzafava, A., Conway, J., Eds.; CRC Press: Boca Raton, FL, 2004; p 317.

(324) Scozzafava, A.; Supuran, C. T. L. Med. Chem. 2002, 45, 284.

(325) Supuran, C. T.; Scozzafava, A. In Protein Misfolding in Neurodegenerative Diseases: Mechanisms and Therpeutic Strategies; Smith, H. J., Simons, C., Sewell, R. D. E., Eds.; CRCPress: Boca Raton, FL, 2008; p 265.

(326) Supuran, C. T.; Scozzafava, A. In The Carbonic AnhvdrasesNew Horizons; Chegwidden, W. R., Carter, N., Edwards, Y., Eds.; Birkhauser Verlag: Basel, Switzerland, 2000; p 197.

(327) Temperini, C.; Scozzafava, A.; Puccetti, L.; Supuran, C. T. Biorg. Med. Chem. Lett. 2005, 15, 5136.

(328) Temperini, C.; Vullo, D.; Scozzafava, A.; Supuran, C. T. $\underline{\text {.Med. }}$ Chem. 2006, 49, 3019.

(329) Temperini, C.; Scozzafava, A.; Vullo, D.; Supuran, C. T. Chemistry 2006, 12, 7057.

(330) Temperini, C.; Innocenti, A.; Scozzafava, A.; Mastrolorenzo, A.; Supuran, C. T. Bioorg. Med. Chem. Lett. 2007, 17, 628.

(331) Temperini, C.; Innocenti, A.; Scozzafava, A.; Supuran, C. T. Bioorg. Med. Chem. 2008, 16, 8373.

(332) Vu, H.; Pham, N. B.; Quinn, R. J. L. Biomol. Screen. 2008, 13, 265.

(333) Innocenti, A.; Scozzafava, A.; Parkkila, S.; Puccetti, L.; De Simone, G.; Supuran, C. T. Bioorg. Med. Chem. Lett. 2008, 18, 2267.

(334) Maresca, A.; Scozzafava, A.; Supuran, C. T. Bioorg. Med. Chem. Lett. 2010, 20, 7255 .

(335) Maresca, A.; Supuran, C. T. Bioorg. Med. Chem. Lett. 2010, 20, 4511.

(336) Lou, Y.; McDonald, P. C.; Oloumi, A.; Chia, S.; Ostlund, C.; Ahmadi, A.; Kyle, A.; Auf dem Keller, U.; Leung, S.; Huntsman, D.; Clarke, B.; Sutherland, B. W.; Waterhouse, D.; Bally, M.; Roskelley, C.; Overall, C. M.; Minchinton, A.; Pacchiano, F.; Carta, F.; Scozzafava, A.; Touisni, N.; Winum, J. Y.; Supuran, C. T.; Dedhar, S. Cancer Res. 2011, 71, 3364.

(337) Cortes, S.; Liao, Z. K.; Watson, D.; Kohn, H. I. Med. Chem. 1985, 28, 601.

(338) Conley, J. D.; Kohn, H. I. Med. Chem. 1987, 30, 567.

(339) Kohn, H.; Sawhney, K. N.; LeGall, P.; Conley, J. D.; Robertson, D. W.; Leander, J. D. I. Med. Chem. 1990, 33, 919.

(340) Kohn, H.; Sawhney, K. N.; Bardel, P.; Robertson, D. W.; Leander, J. D. I. Med. Chem. 1993, 36, 3350.

(341) Bardel, P.; Bolanos, A.; Kohn, H. I. Med. Chem. 1994, 37, 4567.

(342) Choi, D.; Stables, J. P.; Kohn, H. L. Med. Chem. 1996, 39, 1907.

(343) Halford, J. J.; Lapointe, M. Epilepsy Curr. 2009, 9, 1.

(344) Davis, R. A.; Innocenti, A.; Poulsen, S. A.; Supuran, C. T. Bioorg. Med. Chem. 2010, 18, 14.
(345) Supuran, C. T. Mol. Diversity 2011, 15, 305.

(346) Maresca, A.; Supuran, C. T. Bioorg. Med. Chem. Lett. 2011, 21, 1334.

(347) Winum, J. Y.; Montero, J. L.; Innocenti, A.; Supuran, C. T. Chem. Biol. Drug. Des. 2009, 74, 636.

(348) Güzel, Ö.; Innocenti, A.; Scozzafava, A.; Salman, A.; Supuran, C. T. Bioorg. Med. Chem. Lett. 2009, 19, 3170.

(349) Asiedu, M.; Ossipov, M. H.; Kaila, K.; Price, T. I. Pain 2010, 148, 302.

(350) Pacchiano, F.; Carta, F.; McDonald, P. C.; Lou, Y.; Vullo, D.; Scozzafava, A.; Dedhar, S.; Supuran, C. T. L.Med. Chem. 2011, 54, 1896. 